\title{
ANALYSIS OF A DEDICATED ROTATION EXPERIMIENT IN TFTR
}

\author{
Prepared by \\ W.M. Stacey \\ Fusion Research Center \\ and \\ Nuclear Engineering Program \\ Georgia Institute of Technology \\ March, 1992
}

\section{DISCLAIMER}

\begin{abstract}
This report was prepared as an account of work sponsored by an agency of the United States Government. Neither the United States Government nor any agency thereof, nor any of their employees, makes any warranty, express or implied, or assumes any leg al liability or responsibility for the accuracy, completeness, or usefulness of any information, upparatus, product, or process disclosed, or represents that its use would not infringe privately owned iights. Reference herein to any specific commercial product, process, or service by trade name, trademark, manufacturer, or otherwise does not necessarily constitute or imply its endorsement, recommendation, or favoring by the United States Government or any agency thereof. The views and opinions of authors expressed herein do not necessarily state or reflect those of the United States Government or any agency thereof.
\end{abstract}

ACKNOWLEDGEMENT: This report was prepared by the author based on the Ph.D. Thesis of G. Pautasso, the research for which was performed under the author's guidance under Grant DE-FG05-87ER51112 from the U.S. Dept. of Energy. The efforts of S.D. Scott in making readily available the experimental data and analysis codes and in guiding the research are gratefully acknowledged. 


\section{ABS'TRACT}

The results and analysis of a well-diagnosed, dedicated rotation experiment in TFTR are presented. Various neoclassical and anomalous theories for momentum transport are described and compared with the experimental data. The gyroviscocity theory is able to predict the measured central toroidal rotation speed, momentum confinement time and radial torque flow profile reasonably well when a poloidal asymmetry factor $\tilde{\Theta}=1.5$ is used. The cold-ion-perpendicular-viscocity theory requires the assumption of an implausibly large number of cold ions in order to predict the magnitude of the experimental torque flow. The ion-temperature-gradient-mode theory, the untrappedparticle-electrostatic-mode theory and the stochastic-magnetic-perturbation theory all predict torque flows that differ greatly in magnitude, radial profile and parametric dependence from the experimental values.

Evaluation of the poloidal asymmetry factor from a first-principle calculation of poloidal density asymmetries supports $\tilde{\Theta} \sim O(1)$ and leads to radial torque flow profiles predicted by the gyroviscous theory that are within a factor of two of the experimental values, but does not support the seperation-of-variables approximation upon which the gyroviscous calculational model is based. Modification of the grroviscous model to remove the seperation-of-variables approximation by including the radial gradient of tha poloidal asymmetries results in near cancellation of the added term with the original term and an underprediction of the magnitude of the torque flow by at least an order of magnitude. 


\section{INTRODUCTION}

With unbalanced neutral beam injection (NBI) in a tokamak, the torque from the neutral beams causes the plasma to rotate up to velocities comparable to the sound speed. It is observed that a sheared velocity profile is established in a short time (tens of milliseconds) and that an exponential-like decay of the velocity follows the beam turn-off. These are manifestations of radial transfer of angular momentum, which results from viscous forces present in the plasma and from the exchange of momentum between the plasma and the external systems, whose investigation is useful for the following reasons. Due to the large difference between proton and electron masses, momentum transport can be studied neglecting electrons and electron-ion coupling, allowing ion transport effects to be isolated. Convection of momentum is usually less important than momentum conduction, and uncertainties in momentum convection, therefore, cause smaller uncertainties in the measured momentum fluxes and the inferred momentum diffusion coefficients. Phenomena which affect momentum transport may also affect particle and energy transport.

An extensive database of rotation speed measurements in tokamaks with unbalanced NBI has been accumulating in the last decade ${ }^{[1]-[23]}$ which spans a broad range of plasma dimensions, densities and temperatures. In most of the early experiments, the data did not provide a complete characterization of the plasma conditions; only the central rotation velocitiy was routinely determined by measuring the Doppler shift of emission lines from impurities present in the plasma center. Charge exchange recombination spectroscopy (CHERS) diagnostics, that allow a simultaneous determination of velocity and ion temperature profiles, have become operative only in the last few years. Radial profiles of momentum diffusion coefficients and of the momentum fluxes can now be inferred and compared with the local of transport predicted by different theories.

The analysis to date of the experimental data has not been able to suggest a simple and universal dependence of the central velocity or of the momentum cunfinement 
time on the experimental parameters. The apparently complex dependence of the momentum confinement properties on the plasma conditions has suggested the need for an examination of the local parametric dependence of momentum fluxes, as well as of the associated global confinement times and rotation velocities, that are predicted by theoretical models.

Several momentum transport theories have been developed to explain the observed momentum confinement times during neutral beam injection and the velocity decay following beam turn-off. Classical and neoclassical perpendicular viscous forces are orders of magnitude smaller than the siowing-down forces acting on the rotating plasma ${ }^{|3|}$. The neoclassical gyroviscous force ${ }^{\mid 24\}}$, which depends on poloidal asymmetries of the rotation velocity and density, is of the proper magnitude to account for the the momentum confinement times observed on several machines $[5],(11),(21),[25]$ when these asymmetries combine to produce a poloidal asymmetry factor of order unity, which suggests poloidal density and velocity asymmetries of order of the inverse aspect ratio, $\epsilon=r / R$. Although there is some evidence suggesting order $\epsilon$ asymmetries for collisional ions, a self-consistent solution for these asymmetries has not been carried out for any of the rotation experiments. Recent works by Ware [26], Hsu and Sigmar ${ }^{[27]}$ and Stacey and Neeley ${ }^{[28]}$ have pointed out some possible new neoclassical mechanisms of momentum transport.

Momentum diffusion coefficients have been derived for anomalous theories based, respectively, upon stochastic magnetic perturbations ${ }^{29 \mid-[31]}$, untrapped particle modes [32], (33], and ion-temperature-gradient-driven modes [34]-[41]. Comparison of the anomalous theories with the experiments has been extensive only for the last theory and has led to conflicting evidence on the role of these modes in determining momentum confinement.

The comparison between theories and experiments has not yet led to a clear understanding of the causes of momentum transport, but a comprehensive comparison has not yet been done. 
A dedicated rotation experiment was conducted in TFTR employing the recently installed CHERS diagnostic ${ }^{42]}$. It is particularly suited for a careful comparison with theoretical predictions. Shots at different values of plasma current, magnetic field, injected beam power and injection direction were made, to study the parametric dependence of local fluxes of momentum and the corresponding global confinement times.

The purposes of this report are to analyze the data of the dedicated TFTR rotation experiment, to document these results, and to compare with the predictions of neoclassical and anomalous momentum transport theories. Since the gyroviscous theory has been found previously to reproduce the experimental momentum confinement times and its parametric dependence in several devices, particular attention will be devoted to the evaluation of the magnitude of the poloidal variation of densities and rotation frequencies that determine the magnitude of the gyroviscous momentum flux.

The report is organized as follows. Section 2 describes the diagnostics used in the TFTR experiment and the definition of thecretical and experimental quantities needed for the analysis of rotation experiments. In Section 3, the neoclassical and anomalous theories of momentum confinement are summarized. Section 4 documents the experimental conditions and the plasma parameters characteristic of the TFTR experiment. In Section 5, the predicted momentum fluxes, momentum confinement times, etc. are compared with the corresponding experimental quantities. Due to the fundamental role of the dens.ty and velocity poloidal variations in determining the magnitude of the gyroviscous torque, experimental data and calculational models for these quantities are presented in sections 6 and 7 . The effect of anomalous forces on poloidal asymmetries is discussed in section 8 , a reformulation of the gyroviscous torque in terms of the calculated asymmetry factors is givon in section 9 , and the poloidal velocities and density asymmetries calculated for TFTR are discussed in section 10. This work is compared with previous work in section 11 and summarized 
in section 12 .

\section{PLASMA DIAGNOSTICS AND DATA INTER- PRETATION}

\subsection{TFTR diagnostics}

During the plasma discharges of the dedicated TFTR rotation experiment, most of the plasma parameters of interest for our analysis were monitored by more than one diagnostic.

The electron density, $n_{e}$, and the electron temperature, $T_{e}$, were continuously measured by the Multichannel Far-Infrared Interferometer (MIRI) ${ }^{|43|}$ and by the Electron Cyclotron Emission (ECE) ${ }^{[44]}$ diagnostic, respectively. An Abel inversion technique was then used for the evaluation of $n_{e}(R, t)$. Both the MIRI $n_{e}(R, t)$ and the ECE $T_{e}(R, t)$ agree quite well (within $10 \%$ ) with the single-time-point measurements of the same profiles by the Thomson Scattering (TS) diagnostic.

The $v_{\phi}$ and $T_{i}$ central values and radial profiles were obtained by the Charge Exchange Recombination Spectroscopy (CHERS) diagnostic ${ }^{[42]}$ after 400 or $900 \mathrm{~ms}$ of NB injection, using a Diagnostic Neutral Beam (DNB) injector to enhance the production of charge-exchange impurity lines (C VI, $5292 \AA)$. Time histories of central toroidal velocity, $v_{\phi}(0)$, and central ion temperature, $T_{i}(0)$, were determined from the Doppler shift of the Ni XXVII resonance line measured by a horizontal X-ray Crystal Spectrometer (XCS; $K_{\alpha}=1.5879 \AA$ ) ${ }^{\mid 45]}$. The good agreement between $v_{\phi}(0)$ as measured by CHERS and by the XCS is shown in Fig. 1. Only the CHERS data will be used in the following analysis.

The impurity concentration was inferred from the intensity of radiation emission measured by the Visible Bremsstrahlung (VB) $|46|$ and by the Pulse Height Analysis (PHA) diagnostics. The $\mathrm{I}^{\prime} B$ radiation, at $5235 \AA$, is measured along a quasi- 
tangential chord. The PHA diagnostic uses a centrally viewing X-ray detector to perform time-dependent measurements of low- and medium- $Z$ impurity concentration. The agreement between the $Z_{\text {eff }}^{V B}$ and the $Z_{\text {eff }}^{P H A}$ was good for co-injection shots, while in counter-injection shots the PHA data lead to values of $Z_{\text {eff }}$ that are $30 \%$ higher than with the VB data. In NBI plasmas, the $Z_{\text {eff }}^{V B}$ data are considered more accurate than the $Z_{\text {eff }}^{P H A}$ data ${ }^{[47]}$ and therefore will be used for the evaluation of the low $\mathrm{Z}$ impurity concentration in this analysis. The metallic impurity content, $Z_{m e t}=Z_{M}^{2} n_{M} / n_{e}$ is evaluated from the PHA measurements and it is considered a rather accurate measurement. The VB and PHA diagnostics did not measure the radial distribution of impurities and could provide only average values of $Z_{\text {eff }}$ and $Z_{m e t}$

The $H_{\alpha}$ Interference Filter Array (HAIFA) diagnostic, viewing the TFTR inner limiter, provides a measurement of the total $H_{\alpha}$ emission at five different poloidal angles, $0-30^{\circ}$ from the midplane, which allows the evaluation of the flux of deuterium from the inner wall ${ }^{48}$ ).

Seven fission detectors, distributed around the TFTR torus, monitored the neutron source strength of the plasma during each shot. [49]

The X-ray Imaging System (XIS) is composed of an array of $\sim 40$ detectors viewing different chords of the plasma above and below the midplane and recording the soft $\mathrm{x}$-ray emission integrated along these chords $[50 \mid$. The local emission is proportional to $\sum_{j} n_{e} n_{j} Z_{j}^{2} \sqrt{T_{e}} \exp \left(-E_{f} / T_{e}\right)$, where $E_{f}$ is the cut-off energy of a Be filter used to reject soft $\mathrm{x}$-rays less than $\sim 2 \mathrm{keV}$. The comparison between the emissions from two chords symmetric with respect to the horizontal midplane can give information about poloidal asymmetries of $n_{e}^{2} Z_{e f f}$ (assuming $T_{e}=\operatorname{const}(\theta)$ ). The error affecting the emissivity measurements is of the order of $10 \%$. 


\subsection{Interpretation of experimental data}

The equations describing particle and momentum balance for species $\mathrm{j}$ - the $\left(1, m_{j} \vec{v}_{j}\right)$ first two moments of the Boltzmann equation - are, respectively ${ }^{[51]}$ :

$$
\begin{aligned}
& \frac{\partial n_{j}}{\partial t}+\nabla \cdot\left(n_{j} \vec{v}_{j}\right)=\mathcal{S}_{j} \\
& m_{j} \frac{\partial}{\partial t}\left(n_{j} \vec{v}_{j}\right)+\nabla \cdot\left(n_{j} m_{j} \vec{v}_{j} \vec{v}_{j}+\vec{\Pi}_{j}\right)+\nabla p_{j}+n_{j} e_{j}\left(\nabla \Phi+\vec{B} \times \vec{v}_{j}\right)=\vec{R}_{j}+\overrightarrow{\mathcal{M}}_{j}
\end{aligned}
$$

where $m_{j}$ and $e_{j}$ are the mass and charge of species $\mathrm{j}, \mathcal{S}_{j}$ represents the particle sources and sinks, $\vec{v}_{j}$ is the fluid velocity, $p_{j}$ is the pressure, $\overrightarrow{\vec{\Pi}}_{j}$ is the viscosity tensor, $\vec{R}_{j}$ is the collisional friction term, $\overrightarrow{\mathcal{M}}_{j}$ represents the external momentum sources and sinks, and $\Phi$ and $\vec{B}$ are the electrostatic potential and the magnetic field.

In order to allow analytical treatment of the above equations, the flux surfaces are assumed circular, that is:

$$
R=R_{0} h, \quad B=B_{0} / h, \quad h \equiv 1+\epsilon_{0} \cos \theta, \quad \epsilon_{0} \equiv r / R_{0} ;
$$

and the plasma properties are considered independent of the toroidal coordinate. The orthogonal coordinate system, $(r, \theta, \phi)$, has unit vectors indicated as $\hat{r}, \hat{\theta}, \hat{\phi}$. This approximation is appropriate for the TFTR plasmas for which the flux surfaces are modestly shifted $\left(\Delta R_{o} / a \leq 15 \%\right)$ and slightly elongated $(\mathrm{k}<1.05)$ circular flux surfaces.

The flux surface average of a quantity ' $\mathrm{x}$ ' is indicated as $\langle x\rangle \equiv \int x h d \theta /(2 \pi)$ and referred to as "FSA". The toroidal component of Eq.(2) describes the balance among the forces that determine the observed tornidal plasma rotation. Multiplying Eq. (2) by $(R \hat{\phi})$, summing over species and flux surface averaging we obtain:

$$
\begin{aligned}
& \sum_{j} m_{j}<R \frac{\partial n_{j} v_{\phi j}}{\partial t}>+\sum_{j}<R \hat{\phi} \cdot \nabla \cdot \overrightarrow{\vec{\Pi}}_{j}>= \\
& <R \mathcal{M}_{\phi}>-\sum_{j} m_{j}<R \hat{\phi} \cdot \nabla \cdot\left(n_{j} \vec{v}_{j} \vec{v}_{j}\right)>
\end{aligned}
$$

Axisymmetry, charge neutrality and $\mathcal{M}_{\phi} \equiv \Sigma_{j} \hat{\phi} \cdot \overrightarrow{\mathcal{M}}_{j}$ have been used. 
It is difficult to measure the difference among the toroidal rotation speeds of the different ion species, since it is comparable to the magnitude of the measurement e. ror. Therefore, for the purpose of interpreting the experimental data, the toroidal velocity is assumed to be the same for each plasma species. Equation (3) then becomes:

$$
R_{0} m_{a v} \frac{\partial}{\partial t}\left(n_{e t h}(r) v_{\phi}(r)\right)+\mathcal{K}_{\phi}(r)=\mathcal{T}_{\phi}(r)-m_{a v} \frac{R_{0}}{r} \frac{\partial}{\partial r}\left(r v_{\phi}(r) \Gamma_{e}(r)\right)
$$

where

- $m_{a v} \equiv \sum_{j} m_{j} n_{j} / \sum_{j} n_{j}$ is the average ion mass. The 1-D transport interpretation code, SNAP ${ }^{[52]}$, that is used in the analysis, considers four ion species (H, D, C and metal impurity) and the fast ions are not included in the summation. $Z_{\text {eff }}$ and $Z_{m e t}$ are assumed to be a constant and, therefore, the impurity densities have the same radial profile as $n_{e}$.

- $n_{e t h}=n_{e}-n_{f i}$, where $n_{f i}$ is the fast ion density. SNAP assumes that fast ions become thermal ions when their energy falls below $3 / 2 T_{i}$.

- $\mathcal{K}_{\phi} \equiv \sum_{j}<R \hat{\phi} \cdot \nabla \cdot \overrightarrow{\vec{\Pi}}_{j}>$ is the viscosity torque density;

- $\mathcal{T}_{\phi} \equiv<R \mathcal{M}_{\phi}>$ is the beam torque density delivered to the thermal plasma, evaluated in SNAP with a numerical solution of the Fokker Planck equation.

The fast ion distribution is calculated assuming no anomalous diffusion of the fast ions, which is consistent with the TFTR experiments [53].

- The last term in Eq.(5) is the convected torque density. SNAP calculates the beam electron source, $\mathcal{S}_{\boldsymbol{b}}$, by evaluating the ionization rate of the beam neutrals, and the wall source, $\mathcal{S}_{w}$, making use of results from the Degas code [48] and of the edge integrated $H_{\alpha}$ emission; then it evaluates the electron flux, $\Gamma_{e}=n_{e} v_{e r}$ by solving the continuity equation to obtain :

$$
\Gamma_{e}=\frac{1}{r} \int_{0}^{r}\left(\mathcal{S}_{b}+\mathcal{S}_{w}-\frac{\partial n_{e}}{\partial t}\right) r^{\prime} d r^{\prime}
$$


The temporal evolution of $n_{j}(R)$ and $v_{\phi j}(R)$ are measured by a variety of diagnostics reviewed in Section 2.1. SNAP computes the configuration of shifted circular flux surfaces consistent with a kinetic analysis of plasma pressure. To make contact with the laboratory coordinates, each flux surface $\psi^{*}$ is associated with the coordinate $r^{*}=\left[R\left(\psi^{*}, 0\right)-R\left(\psi^{*}, \pi\right)\right] / 2$. For the purpose of obtaining radial profiles of the plasma parameters:

- $\Omega_{\phi} \equiv v_{\phi} / R$ and $T_{i}$ are assumed constant on a particular magnetic surface and only the data points outside the magnetic axis, $r>R_{o}$, are used (because the points on the inside have larger error bars);

- the $n_{e}(r)$ and $T_{e}(r)$ profiles are commonly obtained using the entire profile: $n_{e}\left(r^{\prime}\right)=n_{e}\left(R_{l}, 0\right)=n_{e}\left(R_{r}, \pi / 2\right)$ and $r^{\prime}=\left(R_{l}+R_{r}\right) / 2$.

The momentum balance for the plasma enclosed by the flux surface ' $r$ ' is obtained by integratiıg $\left(E q \cdot(5) \times(2 \pi)^{2} R r\right)$ over the interval [0-r], providinig the definition for the experimental conductive torque flux as a function of measured plasma parameters:

$$
K_{\phi}^{e x}(r)=(2 \pi)^{2} R_{0} \int_{0}^{r} \mathcal{K}_{\phi}\left(r^{\prime}\right) r^{\prime} d r^{\prime} \equiv T_{\phi}(r)-K_{\phi}^{c v}(r)-\dot{Y}_{\phi t h}(r)
$$

where:

- $T_{\phi}(r)=\left(2 \pi R_{0}\right)^{2} \int_{0}^{r} \mathcal{M}_{\phi} r^{\prime} d r^{\prime}$ is the total torque input in the region $[0, \mathrm{r}]$;

- $K_{\phi}^{c v}(r)=\left(2 \pi R_{0}\right)^{2} m_{a v} r v_{\phi}(r) \Gamma_{e}(r)$ is the convective torque flux across the flux surface ' $r$ ';

- and $Y_{\phi x x}(r)=\left(2 \pi R_{0}\right)^{2} \int_{0}^{r} m_{a v} n_{e x x} v_{\phi} r^{\prime} d r^{\prime}$ is the thermal $(x x=t h)$ or total $(x x=t o t)$ plasma angular momentum stored in the region $[0, r]$.

Since SNAP is a steady state code and the analysis of the TFTR parameters will be limited to quasi-steady-state plasma conditions, the term $\dot{Y}_{\phi t h}$, in Eq.(7), vanishes. 
The experimental conductive momentum confinement time, for the region [0-r], is then defined as the momentum replacement rate:

$$
\tau_{\phi x x}^{e x}(r) \equiv \frac{Y_{\phi x x}^{e x x}(r)}{T_{\phi}(r)-\dot{Y}_{\phi x x}^{e x}(r)}
$$

With density and velocity profiles of the form:

$$
x(r)=x(0)\left(1-(r / a)^{2}\right)^{\alpha_{x}}
$$

in steady state conditions, one derives:

$$
\tau_{\phi x x}^{e x}(a)=\frac{m_{a v} n_{e x x}(0) v_{\phi}(0) 2\left(\pi a R_{0}\right)^{2}}{T_{\phi}(a)\left(1+\alpha_{n}+\alpha_{v}\right)}
$$

The appropriate definition of a corresponding theoretical momentum confinement time is then suggested by Eqs. (7) and (2), with $K_{\phi}^{e x}$ replaced by $K_{\phi}^{\text {theo }}$ :

$$
\tau_{\phi x x}^{\text {theo }}(r)=\frac{Y_{\phi x x}(r)}{K_{\phi}^{\text {theo }}(r)+K_{\phi}^{c v}(r)}
$$

Since it is experimentally observed that $K_{\phi}^{c v}(r) \ll K_{\phi}^{e x}(r)$, the convective torque flux is sometimes neglected in the above definition, Eq.(11).

\section{THEORIES FOR MOMENTUM TRANSPORT}

\subsection{Theories of neoclassical momentum transport}

\subsubsection{Gyroviscosity}

The lower limit to momentum transport is that described by the neoclassical theory. When Braginskii's stress tensor is extended to include toroidal effects ${ }^{[24]}$, the toroidal component of the viscous torque, $\langle R \hat{\phi} \cdot \nabla \cdot \overrightarrow{\vec{\Pi}}\rangle$, reduces to the sum of the perpendicular viscosity contribution:

$$
<R \hat{\phi} \cdot \nabla \cdot \overrightarrow{\vec{\Pi}}_{j}^{1,2}>=<\frac{1}{R h_{\theta}} \frac{\partial}{\partial l_{\psi}}\left(R^{3} h_{\theta} \eta_{2 j} \frac{\partial \Omega_{\phi j}}{\partial l_{\phi}}\right)>
$$


and the gyroviscosity contribution:

$$
<R \hat{\phi} \nabla \cdot \overrightarrow{\vec{\Pi}}_{j}^{3,4}>=-<\frac{1}{R h_{\theta}} \frac{\partial}{\partial l_{\psi}}\left(R^{3} h_{\theta} \eta_{4 j} \frac{\partial \Omega_{\phi j}}{\partial l_{\theta}}\right)>
$$

where $h_{x}$ and $l_{x}$ are the metric and length elements in a $(\psi, \theta, \phi)$ flux surface coordinate system.

The neoclassical perpendicular viscous torque of Eq.(12) long has been recognized to be one-to-two orders of magnitude smaller than is necessary to account for measured momentum confinement times ${ }^{22}$. Stacey and Sigmar $\left.{ }^{[24}\right\}_{\text {noted that }}$ if the poloidal asymmetry in density or rotation velocity was order $\epsilon=r / R$, then the neoclassical gyroviscous torque of Eq.(13) was the correct magnitude to account for measured momentum confinement times. The experimental and theoretical evidence for $O(\epsilon)$ poloidal asymmetries was summarized in Refs. [54], [55] and [56].

In the circular flux surface approximation, Eq.(13) for the gyroviscous torque becomes:

$$
<R \hat{\phi} \cdot \nabla \cdot \overrightarrow{\vec{\Pi}}_{j}^{3,4}>\equiv \frac{1}{r} \frac{\partial}{\partial r}<r R \Pi_{\phi \psi j}^{3,4}>=-\frac{1}{r} \frac{\partial}{\partial r}<\eta_{4 j} R^{2} \frac{\partial \Omega_{\phi j}}{\partial \theta}>
$$

Approximating

$$
x(r, \theta) \equiv x_{o}(r)[1+\tilde{x}]=x_{o}(r)\left[1+\tilde{x}, \sin \theta+\tilde{x}_{c} \cos \theta\right]
$$

$\left(x=n_{j}, v_{\phi j}\right)$ and assuming $\tilde{x}$ to have a weak radial dependence (i.e. setting $\partial \tilde{x} / \partial r=$ 0 ) led ${ }^{[24]}$ to the following approximation for the gyroviscous torque:

$$
<R \hat{\phi} \cdot \nabla \cdot \overrightarrow{\vec{\Pi}}_{j}^{3,4}>\equiv-\frac{1}{r} \frac{\partial}{\partial r}<\eta_{4 j} R^{2} \frac{\partial \Omega_{\phi j}}{\partial \theta}>\simeq-\frac{1}{2} \tilde{\Theta}_{j} \frac{r}{R} \frac{\partial}{\partial r}\left(\eta_{4 j o} v_{\phi j o}\right) G
$$

where

$$
\tilde{\Theta}_{j} \equiv \frac{\tilde{v}_{j s}}{\epsilon}\left(4+\frac{\tilde{n}_{j c}}{\epsilon}\right)+\frac{\tilde{n}_{j s}}{\epsilon}\left(1-\frac{\tilde{v}_{j c}}{\epsilon}\right)
$$

and with $G(r) \equiv-r /\left(\eta_{4 j} v_{\phi j}\right) \frac{\partial}{\partial r}\left(\eta_{4 j} v_{\phi j}\right)$. 
Expressions for the momentum confinement time and central rotation speed which took into account radial profiles were developed [21] using the approximation of Eq.(16) and setting $G=1$ :

$$
\begin{aligned}
\tau_{\phi}^{g \nu}(a) & =\frac{\sum_{j} \int_{0}^{a}<R m_{j} n_{j} v_{\phi j}>r d r}{\sum_{j} \int_{0}^{a}<R \hat{\phi} \cdot \overrightarrow{\tilde{\Pi}}_{j}>r d r} \simeq \frac{2 e B R^{2} \hat{Z}}{\tilde{\Theta} T_{i}(0)} \frac{h_{n v T}}{h_{n v}} \frac{\bar{m}_{D}}{m_{D}} \\
v_{\phi}^{g \nu}(0) & \equiv \frac{T_{\phi}(a)}{\sum_{j}\left(j_{0}^{a}\left[R \hat{\phi} \cdot \nabla \cdot \overrightarrow{\tilde{\Pi}}_{j}>+\frac{\partial}{\partial t}<R m_{j} n_{j} v_{\phi j}>j r d r\right) / v_{\phi j}(0)\right.} \\
& =\frac{2 e B T_{\phi}(a) h_{n v T} \hat{Z}}{V_{p} m_{D} \tilde{\Theta} n_{e}(0) T_{i}(0)\left(1+f \tau_{\phi}^{g v}\right)}
\end{aligned}
$$

An effective gyroviscous momentum flux was later constructed ${ }^{[57)}$ by using an effective $\bar{\Theta}$ that was independent of position:

$$
\Pi_{\phi \psi j}^{3.4}(r) \equiv \frac{K_{\phi j}^{g v}(r)}{r R^{2}(2 \pi)^{2}}=-\frac{\bar{\Theta}_{j}}{2 r R^{2}} \int_{0}^{r}\left[r^{\prime} \frac{\partial}{\partial r^{\prime}}\left(\eta_{4 j} v_{\phi j}\right)\right] r^{\prime} d r^{\prime}
$$

Here, the quantities $h_{x y}$ are radial integrals involving the profiles of the quantities ' $x$ ' and ' $y$ ', the last term in Eq.(18) is a mass factor that is unity in deuterium plasmas, $\tilde{\Theta}$ is an effective value, $V_{p}$ is the plasma volume and

$$
f=\frac{\dot{N}_{e}}{N_{e}}+\frac{\dot{v}_{\phi}}{v_{\phi}}
$$

In order to obtain the effective gyroviscous torque for the plasma, Eq.(16) must be summed over ion species. Since the concentration of various ion species is usually unknown, some model is necessary to obtain an expression that can be evaluated from experimental parameters. The simplest model ${ }^{25]}$ is to assume that the plasma consists of a single effective species with charge $Z=Z_{\text {eff }}$ and $m_{p} n_{p}=\sum_{j} m_{j} n_{j} \simeq$ $m_{D} n_{e}$, which leads to

$$
\hat{Z}=Z_{\text {eff }}
$$

in Eqs. (18) and (19). A …sul model 21 results from assuming that a single dominant impurity determums iwith $7,1 /$ and the total gyroviscous torque. In this 
case, charge neutrality and the definition of $Z_{\text {eff }}$ can be used together with Eq.(16) to obtain

$$
\hat{Z}=Z(Z-1) /\left(Z_{\text {eff }}-1\right)
$$

This last model can be extended to include a metal impurity, with $Z_{e f f}^{m e t} \equiv Z_{m e t}$, in which case

$$
\hat{Z}=\bar{Z} \equiv Z(Z-1) /\left(Z_{\text {eff }}-Z_{m e t}-1\right)
$$

\subsubsection{Cold ions theory}

Ware [26] pointed out that the magnitude of the perpendicular viscous torque of Eq.(12) can be enhanced by an excess of cold ions in an ion-impurity plasma. Recycling at the wall produces low energy ions with about $1 \mathrm{eV}$ energy, a factor of 20-50 times less than the local $T_{i}$. The expectation for the presence of rotating cold ions in the plasma bulk relies on the different temperature dependence of the energy equilibration time, $\nu_{E j} \propto n_{h} / T_{h}^{3 / 2}$, and the momentum exchange time, $\nu_{\phi j} \propto n_{I} Z_{I}^{2} / T_{c}^{3 / 2}$, which allows the cold ions to acquire momentum faster than internal energy (the subscripts ' $c$ ' and ' $h$ ' refer to hot' and cold' respectively.)

The starting point for the development of Ware's theory is the neoclassical expression for the radial flux of toroidal momentum for species $j$ :

$$
\Pi_{\phi \psi j}=-\int \frac{d \theta}{2 \pi} \int m_{j} v_{\|} \frac{\mu B+v_{\|}^{2}}{\omega_{c j} R} \sin \theta \tilde{f}_{j s} d^{3} v
$$

where $\tilde{f}_{j}$ is the part of the distribution function which varies as $\sin \theta$ and is odd in $v_{\|}$, given by:

$$
\beta \frac{v_{\|}}{i} \frac{\partial \tilde{f}_{j s}}{\partial \theta}=\sum_{k}\left[C_{j k}\left(\tilde{f}_{j c}, f_{k 0}\right)+C_{j k}\left(\tilde{f}_{k c}, f_{j 0}\right)\right]
$$

Indicating the main ion distribution function as $f_{i}=f_{h}+f_{c}$, where, for simplicity, $f_{h}$ and $f_{c}$ are assumed Maxwellians, and proceeding from the kinetic equations, Ware derived the following expression for the viscous torque:

$$
K_{\phi}^{\text {cold }}(r)=-3.3 m_{\mathrm{i}} n_{\mathrm{c}} \nu_{c l}\left(\epsilon \rho_{l \in}\right)^{2} \frac{\partial v_{\bullet}}{\partial r} \mathcal{F} r\left(2 \pi R_{0}\right)^{2}
$$


with:

$$
\nu_{c I}=\sqrt{2 \pi} \frac{4}{3} \frac{n_{I} e^{4} Z^{2} \ln \Lambda}{\sqrt{m_{i}} T_{c}^{3 / 2}} \quad y=\frac{m_{i}}{m_{I}} \frac{T_{I}}{T_{c}}
$$

and

$$
\mathcal{F}(y)=\frac{1}{(1+y)^{3 / 2}} \frac{1-Z_{I}\left(\frac{m_{i}}{m_{I}}\right)^{2}+\left(\frac{2}{5}+\frac{2}{5} Z_{I} \frac{m_{i}}{m_{I}}\right) y-\left(\frac{3}{5}+\frac{2}{5} Z_{I} \frac{m_{i}}{m_{I}}\right) \frac{m_{i}}{m_{I}} \frac{1}{y}}{1+y}
$$

Eq.(27) can be rewritten as:

$$
K_{\phi}^{\text {cold }}(r)=\mathcal{A} \frac{n_{c}}{n_{e}} y^{3 / 2} \mathcal{F}(y)
$$

witk

$$
\mathcal{A}=3.33 m_{i}\left(\frac{m_{I}}{m_{i}}\right)^{3 / 2} n_{e}\left(\nu_{c I} T_{c}^{3 / 2}\right) \frac{\left(\epsilon \rho_{I \theta}\right)^{2}}{T_{I}^{3 / 2}}\left(-\frac{\partial v_{\phi}}{\partial r}\right)\left(2 \pi R_{o}\right)^{2} r
$$

$\mathcal{A}$ is a function of the plasma parameters that are known from the experimental measurements.

In the TFTR plasma, the main light impurity is carbon $\left(Z_{\text {eff }}=2-4\right)$ and the metal impurities are $\mathrm{Ni}, \mathrm{Fe}, \mathrm{Cr}$, with a $Z_{m e t}=0.2-0.8$. Assuming carbon $\left(Z_{C}=6\right.$, $\left.m_{C}=12 m_{p}\right)$ or iron $\left(Z_{F e}=24, m_{F e}=55.8 m_{p}\right)$ as the main impurity, Eq.(30) becomes:

$$
K_{\phi}^{\text {cold }}(r) \simeq \mathcal{A} \frac{n_{c}}{n_{e}} \frac{1}{\left(1+\frac{m_{I}}{m_{i}} \frac{T_{c}}{T_{I}}\right)^{3 / 2}} \equiv \mathcal{A} \gamma_{c}
$$

\subsubsection{Highly Collisional Theories}

Hsu-Sigmar [27] and Stacy-Neeley [28] have developed theories applicable to highly collisional ions. Since the ions in these TFTR experiments are not highly collisional, we will not discuss these theories. However, we note that these theories exhibit the same plasma paramete: dependence as the gyroviscous theory and, under similar assumptions, lead to the same formulation as given by Eqs. (18)-(20), albeit with different definitions of $\tilde{\Theta}$. 


\subsection{Theories of anomal us momentum transport}

\subsubsection{Stochastic magnetic perturbations}

Morozov and Pogutze [31],29] proposed an anomalous viscosity mechanism based on small-scale turbulence. They derived a self-consistent set of equations (continuity, generalized vorcex, generalized Ohm's law and heat transport equations) for the description of nonlinear low-frequency electromagnetic os sillations in plasmas and the associated transport processes. These equations were obtained for a non-collisional, cylindrical plasma; included nonuniformity of current, pressure and rotation; and were used to evaluate the ciifferent fluxes across surfaces moving with the plasma to determine the transport coefficients. The viscosity coefficient was found to be related to the electron thermal diffusivity as (for $\frac{m_{e}}{m_{i} \beta_{e}}<1$ ):

$$
\chi_{\phi}^{s m p} \simeq \chi_{e}^{s m p} \frac{m_{i} \beta_{e}}{m_{e}}=C_{1} \frac{c^{2} v_{e}}{\omega_{p e}^{2} q R} \epsilon^{\gamma} \frac{m_{i} \beta_{e}}{m_{e}} \propto \frac{A_{i} \epsilon^{\gamma} \eta_{i}^{1.5}}{q R_{o} B^{2}}
$$

where $C_{1}$ is a constant of $\mathrm{O}(1), \gamma=1-2, \epsilon=r / R_{o}, \beta_{e}=\frac{n_{e} T_{c}}{B^{2}\left(2 \mu_{o}\right)}, A_{i}=m_{i} / m_{p}$, and $m_{i, p, c}$ are the ion, proton and electron masses. We can construct a corresponding torque flow to compare with the experiments:

$$
K_{\phi}^{s m p}(r)=(2 \pi R)^{2} r m_{D} n_{e}(r) \chi_{\phi}^{s m p} \frac{\partial}{\partial r} v_{\phi}(r)
$$

Morozov et al. ${ }^{[32]}$ have recently pointed out that the oscillation spectra seen experimentally have a maximum at $k_{\perp} \rho_{i} \sim 1$. and, according to the theory, should not contribute to the transport.

\subsubsection{Ion Temperature Gradient Modes}

The ion-temperature-gradient (ITG) driven modes are electrostatic drift waves driven unstable by an ion-pressure gradient. Their presence requires a threshold condition $\eta_{i} \equiv \frac{d l n\left(T_{1}\right)}{d l n\left(n_{1}\right)}>\eta_{i}^{c r} \sim 1-2$, and implies $\chi_{i}=\chi_{\phi}$ increasing with $\eta_{i}$.

The evaluation of the momentum flux due to electrostatic drift waves, that is: $|35|$

$$
m, n, \chi_{\phi} \frac{\partial u^{\prime} \phi}{\partial r}=-m_{j} n_{j}<\tilde{v}_{r j} \bar{v}_{\phi}>=m_{j} n_{j} \sum_{k^{\prime}}<k_{\nu}^{\prime} \tilde{\Phi}_{k^{\prime}} \tilde{v}_{\|-k^{\prime}}>
$$


( $k^{\prime}$ is the wavelenght number, $<>$ indicates here the time average, $\tilde{x}$ is the fluctuating component of $x$ ) requires the determination of the oscillation spectra and saturation levels of the fluctuations. There exist different approaches for modelling the plasma parameter fluctuations, and for this reason the ITG modes theory is still in evolution:

The extension to a subsonic rotating plasma ${ }^{|35|}$ of the earlier nonlinear theory [36), developed for sheared slab geometry and focused on the lowest radial eigenstate of the system, leads to the the following expression for the turbulent momentum diffusivity:

$$
\chi_{\phi}^{\eta_{i} s s}=\chi_{i}^{\eta_{i} s s}=1.3 \frac{\rho_{s}^{2} c_{s}}{L_{s}}\left[\frac{1+\eta_{i}}{\tau}+\left(\frac{L_{n}}{2 c_{s}} \frac{d v_{\phi}}{d r}\right)^{2}\right]^{2}
$$

The meanings of the new symbols are: $\tau=T_{e} / T_{i}, L_{s}=\frac{q^{2}}{\epsilon} \frac{d q}{d r}-1, L_{n}=n_{e} \frac{d n_{s}}{d r}-1$, $c_{s}=\sqrt{\frac{T_{e}}{m_{i}}}$ and $\rho_{s}=c_{s} \frac{e B}{m_{i}}$ :

A slightly different expression for $\chi_{i}$, less dependent on $\eta_{i}$, has also been derived for toroidal geometry ${ }^{[37]}$ :

$$
\chi_{i}^{\eta_{i} t o r} \simeq \omega_{* e} \rho_{s}^{2} \frac{L_{s}\left(1+\eta_{i}\right)}{R \tau} \propto \frac{\left(1+\eta_{i}\right)}{L_{n} / L_{s}} \sqrt{A_{i}} \frac{\sqrt{T_{e}} T_{i}}{R B^{2}}
$$

The role of higher radial eigenstates in sonic ITG driven turbulence was explored in Ref. [37], and it was concluded that $\chi_{i}$ is enhanced beyond estimates based on the lowest eigenstate alone.

The above formulas for the ion momentum and thermal diffusivities were derived assuming: one-fluid description of the ions; fully developed turbulence, that is $\eta_{i} \gg$ $\eta_{i}^{\text {cr }} ;$ and $L_{n}, \eta_{i} \ll \infty$. The first assumption makes a simulation of the TFTR results questionable since the fast ion population is a large fraction of the total one.

The argument that a large diffusion coefficient should flatten the temperature and velocity profiles, and hence reduce transport, has motivated recent works by Romanelli ${ }^{|39|}$ and Hahm ${ }^{\mid 40}$ focused on the derivation of an expression for $\eta_{i}^{c r}$ as a function of the plasma parameters. Romanelli's expression for $\eta_{i}^{\text {cr }}$ is $|39|$ :

$$
\eta_{i}^{R o m}=(1 .+1 . / \tau) 2 \mathrm{~L}_{n} / R_{o}
$$


and H, Hhm and Tang derived ${ }^{40)}$ :

$$
\eta_{i}^{H \& T}=2 \frac{r-b_{s}}{\tau+b_{s}}+1.88 \frac{L_{n_{i}}}{L_{s}}\left(1+\frac{1}{\tau}\right), \quad b_{s}=k_{\theta}^{2} \rho_{s}^{2}
$$

( $k_{\theta}$ being the poloidal wavele1.gth)

Near threshold, the use of Horton's expression for $\chi_{i}$, based on toroidal quasilinear theory,

$$
\chi_{i}^{\eta_{i} H}=\sqrt{\eta_{i}-\eta_{i}^{c r}} \frac{\rho_{s}^{2} c_{s} L_{s}}{L_{n} R_{o}} \propto \sqrt{\eta_{i}-\eta_{i}^{c r}} \frac{L_{s}}{L_{n}} \sqrt{A_{i}} \frac{T_{e}^{1.5}}{R_{o} B^{2}} \quad \eta_{i} \geq \eta_{i}^{c r}
$$

was recommended in Ref. [58]. The torque flow, in the form given by Eq.(34), will be computed using $\chi_{\phi}^{\eta_{i}} \sim \chi_{i}^{\eta_{i} H}$ and Romanelli's expression for $\eta_{i}^{c r}$ for the purpose of the comparison with TFTR data.

In a recent article, Mattor and Diamond ${ }^{\mid 41\}}$ derived an expression for $\chi_{i}^{\eta_{i}}$ valid in the range $0.95 \leq \eta_{i} \leq 0.95+\left(1+1 / \tau^{\prime} L_{n} / L_{s} \equiv \eta_{i}^{M \& D}\right.$ :

$$
\chi_{i}^{M \& D}=N_{t h} \frac{(1+1 / \tau)^{2}}{2 \sqrt{\pi}}\left(\frac{L_{T}}{L_{s}}\right)^{2} \frac{\rho_{i}^{2} v_{t h i}}{L_{s}}
$$

(with $N_{\text {th }}$ a threshold function dependent on $\eta_{i}$ ).

\subsubsection{Anomalous viscosity due to trapped and untrapped particle modes}

Osipenko et al. ${ }^{|33|}$ have studied ion transport due to interaction of ions with the electrostatic part of the drift oscillations in collisionless, rotating tokamak plasmas, using a Hamiltonian formalism. The total momentum flux is defined by:

$$
\begin{aligned}
& m_{i} n_{j} v_{r i}^{u p} v_{\|}-m_{i} n_{i} \chi_{\phi i}^{u p} \frac{\partial v_{\|}}{\partial r} \\
& =-\sum_{i, \omega} \int d \vec{J} d \vec{\theta} \frac{D_{o}}{2 m_{i}^{2}(2 \pi)^{2} r R} \vec{l} \cdot \frac{\partial f_{i 0}}{\partial \vec{J}} \vec{l} \cdot \frac{\partial B_{t} r^{2}}{\partial \vec{J}} \delta\left(B_{t} r^{2}-\left[\int B_{t} r^{2} f_{i} d^{3} v\right]\right)
\end{aligned}
$$

where $\vec{J}$ and $\vec{\theta}$ are the action-angle variables, $\vec{l}$ is the vector of integers corresponding to the Fourier harmonics in the angular space $\theta$, and $D_{o}$ is a diffusion coefficient describing the evolution of the lower order distribution function $f_{10}$. The spectrum 
used is a model spectrum which agrees with experimental observation: a gaussian spectrum with $\delta \omega \sim \sqrt{2 T_{e} / m_{i}} / L_{n}$.

They found that the contribution of trapped ions to diffusion and heat conduction is $\sqrt{\epsilon}$ times lower than that of passing particles, although the main contribution to electron transport comes from the trapped electrons. The viscosity coefficient due to untrapped particles (up), as derived by them, is

$$
\chi_{\phi}^{u p}=.083 \frac{\rho_{s}}{L_{n}} \frac{T_{e}}{e B} \frac{T_{e}}{T_{i}} \propto \sqrt{A_{i}} \frac{T_{e}^{2.5}}{T_{i}} \frac{1}{L_{n} B^{2}}
$$

and the corresponding trapped particle coefficient is $\rho_{s} / a$ times smaller. The corresponding torque flow is of the form given by Eq.(34).

\section{EXPERIMENTAL DATA}

The experiment consisted of a series of co-, counter- and balanced NBI shots at different plasma currents $\left(I_{p}=1.1\right.$ and $\left.I_{p}=1.7,1.8 \mathrm{MA}\right)$, with a total injected beam power, $P_{b}$, ranging from 4 to $14.5 \mathrm{MW}$. The injection of deuterium neutral beams into a well conditioned deuterium target plasma lasted 0.5 or 1 seconds. Other plasma parameters, which remained fixed, were: minor radius, $a=0.79 \mathrm{~m}$; major radius, $\mathrm{R}=2.45 \mathrm{~m}$; maximum beam energy, $E_{b} \simeq 100 \mathrm{keV}$; beam tangency radii ranging from 1.74 to $2.99 \mathrm{~m}$; beam species mix by neutral current fraction, 45:28:26 (1:1/2 : $1 / 3$ ), and magnetic field, $B_{t}=4.75 \mathrm{~T}$. Also a magnetic field scan was performed at $B_{t}=2.9,3.8$ and $4.8 \mathrm{~T}$, at $I_{p}=1.0 \mathrm{MA}$, using $7 \mathrm{MW}$ of co-beam or $12 \mathrm{MW}$ of counter-beam power.

The experimental conditions, at the time of the diagnostic neutral beam (DNB) injection, at which time the plasma parameters had reached quasi steady state values, are summarized in Table 1. The new parameters listed in this Table are: $\bar{n}$ is the line average density; $\mathcal{P}_{x} \equiv x(0) /[x]$ is the peakedness factor for the plasma parameter $\mathrm{x}$, where $[x]$ is the volume average of $x(r) ; Z_{m e t}=n_{M} Z_{M}^{2} / n_{e}$, where "M" denotes a 
metallic impurity; $L_{\phi f i} / L_{\phi t o t}$ and $W_{f i} / W_{t o t}$ are the fraction of angular momentum and energy, respectively, stored in the fast ions.

Time traces of several of the measured parameters are shown in Fig. 2 for a coand a counter-injected discharge. The co-injected discharge had a lower density and higher ion temperature, stored energy and neutron emission rate than the counterinjected discharge, for the same NB power. The rotation speed was greater for cothan counter-injection.

This set of uni-directional discharges, designated as Hot-Ion-Mode discharges, showed characteristics between the L-mode and the Supershot discharges, that is: low recycling, low ohmic target density (typically $1.2-1.510^{19} \mathrm{~m}^{-3}$ ), density peakedness $\mathcal{P}_{n_{e}}=1.7-2, \tau_{E}$ up to twice the L-mode value, a large fast ion population $\left(n_{f i}(0) / n_{e}(0)=0.1-0.4\right)$ and a relatively high ion temperature $\left(T_{i}(0)=10-20 \mathrm{keV}\right)$. Higher $\mathcal{P}_{n_{e}}$ is usually accompanied by higher $\tau_{E}$.

The rotation data taken in this experiment are characterized by the plot of central rotation velocities shown in Fig. 3 and by the radial profile of rotation velocity-tothermal velocity shown for deuterium and the principal carbon impurity in Fig 4. The collisionality parameters for deuterium and carbon are also shown as a function of raciins in Fig. 4.

Central rotation velocities up to $5.7 \times 10^{5} \mathrm{~m} / \mathrm{s}$ were obtained in the co-injection, low current shots. For the same value of injected torque per particle, the central velocity is $10-20 \%$ lower in counter-injection shots. There does not appear to be a plasma current dependence of the rotation velocity. Most of the $v_{\phi}(0)$ data points show a linear dependence on $T_{\phi}(a) / \bar{n}_{e}$, with the exception of the low-B shots, which also exhibit the lowest densities, are strongly beam dominated, and have a very peaked velocity profile.

The peakedness of the velocity profile, $\mathcal{P}_{v_{\varphi}}=3-6.5$, increases with $I_{p}$ and is less for counter- than co-injection. Characteristic velocity profiles are shown in Fig. 5 The lines in the $t_{\phi}$ and $T_{1}$ pluss are nut a fit of the experimental data but represent a 
mapping of the data for $R>R_{0}$ onto flux surfaces performed by SNAP. Compariscin of the data points for $R<R_{0}$ with the mapping thus provides an indication of any in-out asymmetry in the data. The $T_{i}$ data seem to be quite uniform (with respect to in-out asymmetry) over the flux surface, as would be expected from consideration of the rapid heat conduction along field lines. The $v_{\phi}$ data, on the other hand, exhibit an in-out asymmetry larger than the experimental error. The symmetry found in the $T_{i}(r)$ data would suggest that the asymmetry in the $v_{\phi}$ data is real.

Torque flow terms have been evaluated for several shots. The convected torque flow, $T_{\phi}^{c v}$, is approximately $1-10 \%$ of the conducted flow, $K_{\phi}^{e x}$, over most of the plasma region, and the total cx losses amount to only a few percent.

The accuracy of the calculated source of torque can be checked with a couple of methods. Since the calculated neutron emission depends on whether the beams are deposited (most of the neutrons are from beam-beam and beam-target interaction), one can compare the calculated and measured neutron rates. Since the fusion cross-section is heavily weighted to high energy, good agreement with measured neutrons provides some confidence that the initial deposition profile is correct, but less about whether the fast ions are well-confined radially. A second check can be done by comparing the total stored energy as calculated by SNAP from density and temperature measurements and calculated fast ions, with the same quantity measured by a diamagnetic loop.

The difference between calculated and measured neutrons was, on average, $+20 \%$ for counter-injection and $-17 \%$ for co-injection shots. The uncertainty affecting the neutron measurements is estimated to be $\sim 12 \%$. The origin of this disagreement, which suggests a systematic error is affecting the measurement procedure or the calculation, has not been clarified yet.

Some discrepancy is also found between the stored energy inferred from magnetic measurements and the one resulting from the SNAP kinetic calculations. The diamagnetic stored energy from magnetic measurements agrees with the calculated value 
to within $\pm 10 \%$.

If the discrepancies between the measured and calculated neutron production rate and stored energy are due to systematic errors in the modeling, this could also affect the torque deposition calculation. The credibility of the SNAP deposition profile is suggested by the good agreement with TRANSP ${ }^{[59]}$ calculations, which employ a more sophisticated deposition and thermalization profile model (particle-following Monte Carlo code).

The thermal momentum confinement times, constructed from Eq.(8) and plotted in Fig. 6, show a modest degradation with $P_{b}$, a significant increase with $I_{p}$ and no clear dependence on the direction of injection. As shown in Table 1 , the peakedness of the velocity profile increases with $I_{p}$, which may be related to the increase of $\tau_{\phi t h}^{e x}$ with $I_{p}$. The total momentum confinement time is $\tau_{\phi t o t}^{e x} \sim 3 \tau_{\phi t h}^{e x}$ (which means that $2 / 3$ of the plasma momentum is carried by the fast ions), and for $\tau_{\phi t o t}^{e x}$ the $I_{p}$ dependence disappears because of the decrease of $n_{f i}$ with increasing $I_{p}$.

Central ion temperatures up to $25 \mathrm{keV}$ were reached in low current, balanced and co-injection shots. For the same value of injected power, $T_{i}(0)$ decreases with $I_{p}$ and is less for counter-injection than for co-injection, consistent with the increase in $\bar{n}_{e}$ with $I_{p}$ and with higher $\bar{n}_{e}$ for counter- than for co-injection. The ion temperature peakedness, $\mathcal{P}_{T_{i}}=2.2-4.8$, increases with $I_{p}$ and $P_{b}$ and is less for counter- than co-injection, similarly to $\mathcal{P}_{\boldsymbol{v}_{\phi}}$.

Typically the electron density increases during the beam heating, reaching an equilibrium value in $\sim 300 \mathrm{~ms} . \bar{n}_{e}$ increases with $P_{b}, I_{p}$ and from co- to counterinjection. The $\bar{n}_{e}$ is $\sim 35 \%$ higher in the high current shots; $\mathcal{P}_{n_{e}(0)}$ does not show a clear $P_{b}$ or $I_{p}$ dependence. Typical $n_{e}$ profiles are shown in Fig. 5 for co- and counter-injection shots.

For the same $I_{p}$ and $P_{b}$, the co- and counter-injection shots have remarkably different density-rise histories and the final $\bar{n}_{e}$ is $10-30 \%$ higher in the counter-shots. A larger density in the proximity of the inner bumper limiter is also a characteristic 
of the counter- density profiles. The rate of rise of $N_{e}$ and the total electron density has been compared to the integrated beam electron source, $N_{b}$, for $14 M W$ co- and counter- shots. During the first $200 \mathrm{~ms}, \partial N_{e} / \partial t$ is approximately equal to the rate of beam electron input, $\partial N_{b} / \partial t$, for the co-injected shots, but it is 1.5-2.5 times higher in the counter-injected shots, suggesting an enhancement of the electron influx from the wall. There is a substantial difference in the magnitude and poloidal profile of the $H_{\alpha}$ signal between co- and counter-injection, suggesting a substantial influence of the direction of injection on the edge conditions.

Charge exchange, first-orbit and shine-thru losses were calculated to determine their contribution to the difference in $\dot{N}_{e}$ between co- and counter-injection. The first orbit losses are higher in counter-injected shots, while charge exchange and shinethru losses are almost independent of direction of injection. But since the final $\bar{n}_{e}$ in counter-shots is 10-30\% higher than it is in co-shots, at fixed $P_{b}$, the calculated total losses differ only by a few percent between co- and counter-injection and cannot account for the different electron density time histories observed in the experiments.

The VB diagnostic was used to determine the density of the light impurities, principally carbon. The metal content, evaluated from the PHA measurements, was small in all discharges (metal contribution to $Z_{\text {eff }}, Z_{\text {met }} \sim 0.3$ ), except for the low current co-injection shots, where $Z_{m e t}=0.6-0.8$. No information was available on the radial distribution of the impurities; therefore $Z_{\text {eff }}$ and $Z_{m e t}$ are assumed constant in our analysis. The PHA value of $Z_{\text {eff }}$ is larger by about $10-30 \%$ for counterthan co-injected shots, which together with the data given in Fig. 2, suggests that the higher $\bar{n}_{e}$ and lower $T_{i}(0)$ for counter- than co-injection could be associated with larger impurity accumulation. However, VB values of $Z_{\text {eff }}$, which are considered more accurate than the PHA values, do not support this explanation.

The thermal energy confinement time was compared with the thermal momentum confinement time. There appears to be a correlation between the two, but there is a significant scatter in the data. To correlation is observed between the total energy 
and momentum confinement times.

\section{COMPARISON OF THEORY AND EXPER- IMENT}

\subsection{Gyroviscous theory}

The gyroviscous theory embodied in Eqs. (18) - (20) was applied to the analysis of the data of the previous section, making use of experimental data to evaluate the parameters entering these formulas. Two different models for summing over ion species to obtain an effective plasma gyroviscous torque were examined, corresponding to the expressions for $\hat{Z}$, given by Eqs. (22) and (24).

\subsubsection{Case $\hat{Z}=\bar{Z} \equiv Z(Z-1) /\left(Z_{\text {eff }}-Z_{\text {met }}-1\right)$.}

A comparison of theoretical (Eq.(19)) and experimental central rotation velocities for all the shots in Fig. 3 is given in Fig. 7, and a similar comparison of theoretical (Eq.(18)) and experimental momentum confinement times is given in Fig. 8. A value $\tilde{\Theta}=1.5$ was found to provide a good fit of the gyroviscous formulas to the data. (The possibility of $O(1)$ poloidal asymmetry factors in TFTR is discussed in the following sections.) The agreement is reasonably good, although there is a general tendency to overpredict $\tau_{\phi}$ for the counter-injection shots and to underpredict it for the co-injected shots. The VB values of $Z_{\text {eff }}$, which were similar for co- and counterinjection were used, to evaluate $\tau_{\phi}^{g v}$. If the PHA values of $Z_{e f f}$, which were larger for counter- than co-injection were used, the predicted values of $\tau_{\phi}^{g v}$ for counter-injection would be reduced relative to the $\tau_{\phi}^{g "}$ for co-injection, improving the agreement with experiment.

Next, we determine if the dependences of the momentum confinement time on $T_{1}(0), \bar{Z}\left(Z_{\text {eff }}\right)$ and $B$ predicted by the gyroviscous theory are consistent with the 
data. It does not suffice to merely plot the experimental $\tau_{\phi}^{e x}$ against each of these variables, because there may be a correlation among the values of two or more of the variables appearing in Eq.(18). To account for the possibility of such correlations, we will divide the experimental $\tau_{\varphi}^{e x}$ by Eq.(18), but with the variable whose dependence is to be checked suppressed, and plot the resulting ratio (or its inverse) against the suppressed variable. If the dependence upon the suppressed variable predicted by Eq.(18) is consistent with the data, then the resulting plot should consist of points all falling on the $45^{\circ}$ line. For example, the ratio

$$
T_{i}^{g v}(0)=\frac{e B R_{o}^{2} \bar{Z}}{\tau_{\phi}^{e x}} \frac{n_{e t h}(0) h_{n v T}}{n_{e}(0) h_{n_{t h} T} \tilde{\Theta}}
$$

should plot as a straight line against $T_{i}(0)$. Similarly the quantities:

$$
\bar{Z}^{g v}=\frac{\tau_{\phi}^{e x} T_{i}(0)}{e B R_{o}^{2}} \frac{n_{e}(0) h_{n_{t h} T} \tilde{\Theta}}{n_{e t h}(0) h_{n v T}}
$$

and

$$
B^{g v}=\frac{\tau_{\phi}^{e x} T_{i}(0)}{e R_{o}^{2} \bar{Z}} \frac{n_{e}(0) h_{n_{t h} T} \tilde{\Theta}}{n_{e t h}(0) h_{n v T}}
$$

should plot as straight $45^{\circ}$ lines against $\bar{Z}$ and $B$, respectively.

The quantities $T_{i}^{g v}(0)$ and $\bar{Z}^{g v}$ are plotted against $T_{i}(0)$ and $\bar{Z}$, respectively, in Figs. 9 and 10 for all the shots in this dedicated experiment. The parametric dependence pyerlicted by Eq.(18) is consistent with the data for $T_{i}(0)$ and not inconsistent with the data for $\bar{Z}\left(Z_{\text {eff }}\right)$, as shown in Figs. 9 and 10, respectively. The parametric dependence on B predicted by Eq.(18) was not evident from the limited set of data points in the $B$ scan.

The torque flow, $K_{\phi}^{g v}$, consistent with the above gyroviscous theory predictions, given by $\mathrm{E}$..$(20)$, is compared with the same quantity constructed from the experimental data in Fig. 11. The comparisons were similar for all shots examined. There is a significant overprediction in the center, but reasonable agreement in the outer regions. The same constant $\tilde{\Theta}=1.5$ was used in this prediction, and the disagreement in shape could indicate a radial dependence of $\tilde{\Theta}$. 


\subsubsection{Case $\hat{Z}=Z_{\text {eff }}$}

Figure 12 shows the comparison between experimental and theoretical central velocity, as predicted by Eq.(19) using the $\hat{Z}=Z_{\text {eff }}$ model. A value of $\tilde{\Theta}=0.4$ was used in this case to obtain a good fit of $v_{\phi}^{g v}(0)$ to the data, which implies the assumptions of $O(0.2 \epsilon)$ poloidal asymmetry factors, $\tilde{\Theta}$. The $v_{\phi}^{g v}(0)$ formula reproduces the trend of variation of the experimental data.

The scatter in the comparison of $\tau_{\phi}^{g v}, T_{i}^{g v}(0), B_{g v}$ and $\bar{Z}$, with the experimental data are larger with the $\hat{Z}=Z_{\text {eff }}$ model than with the $\hat{Z}=Z(Z-1) /\left(Z_{\text {eff }}-Z_{\text {met }}-1\right)$ model for summing over ion species. This suggests the dominance of impurities in determining the gyroviscous torque.

\subsection{Cold ions theory}

In order to evaluate Ware's expression for the torque flow, Eq.(27), the cold ion density and temperature must be known. Since neither theoretical expressions nor measurements of these quantities are available, we proceeded by determining the magnitude of the ratio $\gamma_{c}$, in Eq.(32), that is required to fit that expression to the experimental data $\left(K_{\phi}^{\text {cold }}(r)=K_{\phi}^{e x}(r)\right)$. The collision frequency $\nu_{c I}$, Eq.(28), has been evaluated assuming carbon $(\mathrm{C})$ and iron ( $\mathrm{Fe}$ ), alternatively, as impurity ' $\mathrm{I}$ '. In both cases, the required value of this ratio $\left(\gamma_{c} \simeq 10^{2}\right)$ is well above any value that is plausible. Similar results were found for the other shots.

\subsection{Anomalous torque flows}

The torque flows, $K_{\phi}\left(r^{\prime}\right.$, constructed from Eq. (34) using the $\chi_{\phi}$ predicted by the various anomalous theories described in Section 3.2 have been compared with the torque flow interpreted from the local momentum balance measurements and constructed according to Eq.(7), for each shot of the rotation experiment. A representative shot was chosen to illustrate the results, which were similar for all shots in the experiment. 
There is a substantial disagreement in radial profile and in magnitude between each of the anomalous theoretical torque flows and the experimental torque flow, as can be seen in Fig. 11. The predicted torque flow due to untrapped particle modes, $K^{u p}$, evaluated by using Eq.(43), severely underestimates (1-2 orders of magnitude) the experimental torque flow everywhere except very near the center of the plasma.

The predicted torque flow due to stochastic magnetic perturbations, $K^{s m p}$, evaluated by using Eq.(33), is in reasonable agreement with experiment near the center of the discharge, but decreases with plasma radius for $r / a \geq 0.5$ and severely underpredicts ( $\sim 2$ orders of magnitude) the experimental torque flow near the plasma edge. $C_{1}=1$ and $\gamma=2$ have been used to evaluate $\chi_{\phi}^{s m p}$.

The ' $\eta_{i} H$ ' torque flow is computed using $\chi_{\phi}^{\eta_{i}}=\chi_{i}^{\eta_{i} H}$, Eq.(40), in conjunction with Romanelli's formula, Eq.(38), for $\eta_{i}^{c r}$. Figure 13 illustrates the radial profiles of $\eta_{i}^{e x}$ and those of several theoretical expressions of $\eta_{i}^{c r}$. For $r \leq 0.1-0.2 m$, the experimental $\eta_{i}$ is smaller than $\eta_{i}^{R o m}$ and the ITG flux vanishes. For $r \geq 0.1-0.2 m$, up to the plasma edge, $\eta_{i}^{e x}$ exceeds the theoretical critical value and the difference $\left(\eta_{i}^{e x}-\eta_{i}^{R o m}\right)$ remains confined in the range $0-1.2$. The ' $\eta_{i} H$ ' momentum flux is absent in the center of the plasma, becomes comparable in magnitude to the experimental flux for $0.3 \leq r \leq 0.5$, and then drops to a small fraction of the experimental flux towards the edge.

The error bars affecting the evaluation of $\eta_{i}$, and subsequently of $\chi_{i}^{\eta_{i}}$, are relatively large. They were estimated by performing a series of 100 transport analysis varying the input plasma parameters within their ranges of uncertainty by Monte Carlo sampling a Gaussian error distribution [17]. The error bars on the computed and measured $\eta_{i}$ ( and on the other figures ) represent the standard deviation of the calculated $\eta_{i}$. The correlation between the experimental and the theoretical $\eta_{i}$ has also been discussed in Ref. [17].

Among the other expressions for the diffusion coefficient discussed in Section 3.2.2, $\lambda_{i}^{\eta_{1} \text { tor }}$, Eq.(37), predicts the smallest diffusion coefficient. The torque flow evaluated 
assuming $\chi_{\phi}^{\eta_{i}}=\chi_{i}^{\eta_{i} \text { tor }}$ in Eq.(34) is indicated by $K_{\phi}^{\eta_{i} \text { tor }}$ in Fig. 11 ; it overestimates the experimental flux by an order of magnitude in the center and underestimates it by a factor of $\sim 2-4$ for $r>0.6 \mathrm{~m}$.

While the different momentum diffusivities based on the ITG theory fail to reproduce the experimental data, $\eta_{i}^{e x} \sim \eta_{i}^{c r}$ over most of the plasma region, both for Romanelli's $\eta_{i}^{\text {cr }}$, Eq.(38) [39], and for Hahm's $\eta_{i}^{\text {cr }}$, Eq.(39) [40) (having assumed $b_{s}=0.1$ ), as shown in Fig. 13. These results would support the hypothesis that ITG transport is regulated by marginal stability. However, more recent experiments have provided firm evidence that ion transport is not regulated by marginal stability [16]. Also the appropriateness of the transport coefficients $\chi_{\phi}^{\eta_{i} \text { tor }}$, Eq.(37), and $\chi_{i}^{\eta_{i} H}$, Eq.(40), which were derived for $\eta_{i}$ well above threshold, are questionable for the experiments analyzed in this paper.

\subsection{Anomalous momentum diffusivities}

Starting from the torque flux, $K_{\phi}^{e x}$ of Eq.(7), interpreted from the local momentum balance measurements, it is possible to construct an experimental momentum diffusivity, but in order to do so it is necessary to make an assumption about the mathematical form for of $K_{\phi}^{e x}$. The most common assumption is that it has the form:

$$
\chi_{\phi}^{e x}(r)=\frac{-K_{\phi}^{e x}(r)}{(2 \pi)^{2} R_{o} r m_{D} \frac{\partial}{\partial r} v_{\phi}}
$$

The experimental $\chi_{\phi}^{e x}$ and the anomalous $\chi_{\phi}$, constructed by using the measured plasma parameters to evaluate Eqs. (33), (37), (40) and (43), were compared. The differences between experimental and predicted values are similar to the differences among the $K_{\phi}$ 's previously discussed.

It is of interest, however, to examine how the parametric dependences predicted by the various theories compare with the experimental data. A regression analysis of $\chi_{\phi}^{e x}$ in the range $0.25<\frac{r}{a}<0.7$ produced the following parametric dependence ${ }^{[17}$ :

$$
\chi_{\phi}^{e x} \propto C B^{0.16} I_{p}^{-0.59} P_{b}^{0.23}\left(\frac{r}{a}\right)^{1.22}
$$


where $\mathrm{C}=1$ and 1.15 for co- and counter-injection, respectively.

A similar regression analysis, using as data points the values of $\chi_{\phi}$ predicted by the different anomalous theories and as variables $\left(B, I_{p}, P_{b}, r / a\right)$ produced a correlation able to fit the experimental data with a standard deviation no better than $40 \%$. This large range of scatter of the data around the fitting curve leads to the conclusion that this set of parameters cannot properly represent the parametric dependence of the theoretical $\chi_{\phi}$ 's.

Since the magnitude of the $\chi_{\phi}^{\text {theo }}$ is comparable to the $\chi_{\phi}^{e x}$ only over a small range of the minor radius, we checked if $\chi_{\phi}^{e x}$ correlates with the following factors, representing the $\chi_{\phi}$ 's given by Eqs. (33), (37), (40) and (43), in the range $r=0.2-0.4, m$ :

$$
\frac{\epsilon^{2} T_{e}^{1.5}}{q R B^{2}}, \quad \frac{\sqrt{T_{e}} T_{i} L_{s}}{R B^{2} L_{n}}, \quad \sqrt{\eta_{i}-\eta_{i}^{c r}} \frac{T_{e}^{1.5} L_{s}}{R B^{2} L_{n}}, \quad \frac{T_{e}^{2.5}}{L_{n} B^{2} T_{i}}
$$

We have been unable to identify any meaningful correlation between measured $\chi_{\phi}$ values and the factors listed above.

The disagreement of the anomalous theories does not exclude the anomalous nature of momentum transport. In fact, while theory has failed to identify the driving forces of the plasma parameter fluctuation, the evaluation of particle and heat flux directly from the fluctuation measurements has given results comparable to the experimental measurements $|60|$. A next step in the comparison of experiments and theory would be the computation of the momentum flux directly from the measured fluctuation spectrum and amplitude.

\section{EXPERIMENTAL EVIDENCE FOR POLOIDAL ASYMMETRIES IN TFTR}

\subsection{Measurements of $n_{e}^{2} Z_{e f f}(\mathbf{R})$}

The $\mathrm{x}$-ray emission intensity measured ${ }^{|61|}$ by the XIS diagnostic for a series of co- and counter-injection shots, both for the $\mathrm{OH}$ and NB phase, show profiles symmetric with 
respect to the midplane, indicating the absence of poloidal asymmetries of $n_{e}^{2} Z_{\text {eff }}$ within the error bar affecting the measurements themselves. The error bar affecting the measurement of each of the $4 \uparrow$ detectors is $\sim 10 \%$. The uncertainty in the overall up-down asymmetry is therefore reduced by a factor of $1 / \sqrt{40}$.

The symmetry of the XIS emission profiles does not rule out compensating asymmetries in the main ion and impurity densities, but implies that a relation among them must be satisfied. When $Z_{\text {eff }}=3, Z_{\text {met }}=0.5, Z_{I}=6$ and $Z_{M}=26$, charge neutrality, the definition of $Z_{\text {eff }}$ and the magnitude of the experimental error in $n_{e}^{2} Z_{\text {eff }}$ would require:

$$
\left|\tilde{n}_{i}+\tilde{n}_{I}+0.2 \tilde{n}_{M}\right|<<0.1
$$

in order for compensating asymmetries to mask an $O(\epsilon)$ carbon asymmetry.

\subsection{Measurements of $\Omega_{\phi I}(R)$}

Figure 5 shows the toroidal velocity radial profile as measured by CHERS for one shot. The in-out asymmetry of $\Omega_{\phi I}(R)$, visible in the plasma center, of $O(\epsilon)$ or larger, was characteristic of most of the measured velocity radial profiles. The $T_{I}(R)$ profiles, measured by the same diagnostic, appear symmetric. The symmetry of the $T_{I}(R)$ profile, which is expected theoretically, seems to rule out the possibility of an error in the evaluation of the plasma center location; it suggests that the the in-out asymmetry of the toroidal rotation is real, or that the errors in the $T_{I}$ and $v_{\phi I}$ data propagate in different ways. The PPPL diagnostıcians do not have confidence in the ability of CHERS to diagnose in-out asymmetries in $T_{I}(R)$ and $\Omega_{\phi I}(R)$, since the measured signal attenuates exponentially with the distance from the plasma edge, due to beam attenuation. 


\section{THEORY FOR POLOIDAL VELOCITY AND DENSITY ASYMMETRIES}

An essentially neoclassical model is used to calculate the poloidal density asymmetries and poloidal velocities. While neoclassical theory is thought to underestimate many aspects of ion transport, parallel neoclassical transport can account fairly well for resistivity, bootstrap current, and damping of poloidal velocity ${ }^{|62|}$. Thus, we are encouraged to use a neoclassical model for the viscocity since the parallel viscocity is the dominant viscocity mechanism entering the poloidal momentum balance. We use a "zero-heat-flux" viscocity model (appendix). We have examined a "finite-heatflux" viscocity model but have found that results were quite sensitive to unknown parameters.

\subsection{Continuity equation and form of the poloidal flow}

By subtracting the FSA of the continuity equation, Eq.( 1), from Eq.( 1), one obtains:

$$
B_{\theta} \frac{\partial}{\partial \theta}\left(\frac{n_{j} v_{\theta j}}{B_{\theta}}\right)=r \mathcal{S}_{j}-\frac{1}{R} \frac{\partial}{\partial r}\left(n_{j} v_{r j} r R\right)-<r \mathcal{S}_{j}-\frac{1}{R} \frac{\partial}{\partial r}\left(n_{j} v_{r j} r R\right)>\left(\equiv \mathcal{C}_{j}\right)
$$

The LHS term of the equation is $\sim\left(\epsilon n_{j} v_{\theta j}\right)$, while the RHS is :

$$
\mathcal{C}_{j} \sim r \mathcal{S}_{j o} \tilde{\mathcal{S}}_{j}-n_{j} v_{r j} \epsilon \cos \theta-\frac{\partial}{\partial r}\left[r\left(\tilde{n}_{j} v_{r j}+n_{j} \tilde{v}_{r j}\right)\right]
$$

The RHS of Eq.(51) is usually negligible with respect to the LHS and the equation in commonly solved to obtain:

$$
\frac{n_{j} v_{\theta j}}{B_{\theta}} \equiv K_{j}[=\operatorname{const}(\theta)]
$$

When the RHS of Eq.(51) cannot be neglected because large poloidal asymmetries of localized sources ${ }^{[63]}$ or a large radial particle flux are present, the proper form of the poloidal velocity becomes:

$$
v_{\theta j}=\frac{B_{\theta}}{n_{j}}\left(K_{j}+\int_{0}^{\theta} \frac{\mathcal{C}_{j}}{B_{\theta}} d \theta\right)
$$


We have estimated the magnitude of the RHS of Eq.(51) from the experimental data. The main (deuterium) ion source is the sum of the NBI source, which dominates the center of the discharge, and of the recycling source, localized near the edge. We conclude that the RHS of Eq.(51) is negligible over the entire plasma region for the main ions. The impurity source is localized at the plasma edge. The magnitude of this carbon source is such that the RHS of Eq.(51) becomes comparable to the magnitude of the term $\left(n_{j} v_{\theta j}\right)$ and cannot be neglected for the carbon impurity, within a few centimeters at the edge. Thus, we conclude that Eq.(53) is a valid representation of the form of the poloidal flow over most of the plasma region, i.e. for $\mathrm{r} / \mathrm{a} \sim 0-0.9$.

\subsection{Radial momentum balance equation and form of the toroidal flow}

The radial projection of the momentum balance equation, Eq.(2), for the particle species $\mathrm{j}$, is:

$$
g_{r j}+\frac{\partial p_{j}}{\partial r}+e_{j} n_{j} \frac{\partial \Phi}{\partial r}+e_{j} n_{j}\left(v_{\phi j} B_{\theta}-v_{\theta j} B_{\phi}\right)-\mathcal{M}_{r j}-R_{r j}+\Pi_{r j}+m_{j} v_{r j} \mathcal{S}_{j}=0(55)
$$

where:

$$
g_{y j} \equiv \hat{y} \cdot\left[m_{j} n_{j}\left(\vec{v}_{j} \nabla\right) \vec{v}_{j}\right], \quad \quad \Pi_{y j} \equiv \hat{y} \cdot \nabla \cdot \overrightarrow{\tilde{\Pi}}_{j}
$$

This equation can be used to write the toroidal velocity as:

$$
v_{\phi j}=\frac{v_{\theta j}}{\beta}-\frac{1}{e_{j} n_{j} B_{\theta}}\left(g_{r j}+\frac{\partial p_{j}}{\partial r}+e_{j} n_{j} \frac{\partial \Phi}{\partial r}+\Pi_{r j}\right)
$$

where the radial friction, momentum input and particle source terms can be neglected because they are several orders of magnitude smaller than the other terms in the equation, for TFTR. The inertia term can be written as:

$$
g_{r j}=-m_{j} n_{j}\left(\frac{v_{\theta j}^{2}}{r}+\frac{v_{\phi j}^{2}}{R} \cos \theta-\frac{1}{2} \frac{\partial v_{r j}^{2}}{\partial r}+\frac{v_{\theta j}}{r} \frac{\partial v_{r j}}{\partial \theta}\right)
$$

and the viscous force, $\Pi_{r j}$, is given in the Appendix. 
We rewrite the toroidal velocity as:

$$
v_{\phi j}=\frac{v_{\theta j}}{\beta}-\frac{1}{B_{\theta}}\left(\frac{1}{e_{j} n_{j}} \frac{\partial p_{j}}{\partial r}+\frac{\partial \Phi}{\partial r}\right)+R \Omega_{\phi j} U_{j}
$$

where:

$$
\begin{aligned}
U_{j} & =U_{j c} \cos \theta+U_{j s} \sin \theta \simeq \\
& \frac{\eta_{0 j}}{e_{j} n_{j} r v_{\phi j} B_{\theta} R}\left\{v_{\theta j}\left[\left(\frac{r}{L_{\eta_{o j}}}+\frac{r}{L_{v_{\theta j}}}\right) \sin \theta+\frac{2}{3} \frac{\vartheta_{j}}{\epsilon}\left(1+\frac{r}{L_{\theta_{j}}}+\frac{r}{L_{\eta_{0 j}}}+\frac{r}{L_{v_{\theta j}}}\right)\right]\right. \\
& \left.+\frac{\beta A_{j} R}{\epsilon}\left(\frac{r}{L_{\eta_{0 j}}}+\frac{r}{L_{A_{j} / r}}-\frac{r}{L_{q}}\right)\right\}+\frac{m_{j}}{e_{j}} \frac{\Omega_{\phi j}}{B_{\theta}} \cos \theta
\end{aligned}
$$

We have defined $L_{x} \equiv-x(d x / d r)^{-1}$.

\subsection{Poloidal variations of $\Omega_{\phi j}$}

The contributions of the poloidal velocity, the pressure and the potential terms to $\tilde{\Omega}_{\phi j}$ can be written, respectively as:

$$
\begin{aligned}
& \tilde{\Omega}_{\phi j\left(v_{\theta}\right)}=-\frac{v_{\theta j 0}}{v_{\phi j 0}} \frac{1}{\beta}\left(\tilde{n}_{j}+2 \epsilon \cos \theta\right) \\
& \tilde{\Omega}_{\phi j(p)}=\frac{T_{j}}{e_{j}} \frac{\tilde{n}_{j(c, s)}}{v_{\phi j 0}} \frac{1}{B_{\theta 0} L_{\tilde{n}_{j(c, s)}}} \equiv \mathcal{V}_{j}^{*} \tilde{n}_{j(c, s)} \frac{a}{L_{\tilde{n}_{j(c, s)}}} \equiv \mathcal{V}_{j(c, s)} \tilde{n}_{j(c, s)}
\end{aligned}
$$

and

$$
\tilde{\Omega}_{\phi j(\Phi)}=-\frac{1}{v_{\phi j 0} B_{\theta 0}} \frac{\partial \Phi_{1}}{\partial r}
$$

where one has written $\Phi(r, \theta)=\Phi_{0}(r)+\Phi_{1}(r, \theta)$ (the subscripts ' 0 ' and ' 1 ' do not refer to the order of magnitude of the functions but, respectively, to the FSA part and to the remaining part of the $\Phi$ function).

The parallel momentum equation for electrons,

$$
\frac{1}{e n_{e}} \frac{\partial p_{e}}{\partial \theta}=\frac{\partial \Phi}{\partial \theta}
$$

can be used to derive:

$$
\frac{\partial \Phi_{1}}{\partial r}=-\frac{T_{e} \tilde{n}_{e}}{e}\left(\frac{1}{L_{T_{e}}}+\frac{1}{L_{\tilde{n}_{e}}}\right)
$$


Alternativelv one can also write:

$$
\Phi_{1}(r, \theta)=\Phi_{0}(r) \tilde{\Phi}(r, \theta) \quad \text { and } \quad \frac{\partial \Phi_{1}}{\partial r}=\frac{\partial \Phi_{o}}{\partial r} \tilde{\Phi}+\frac{\partial \tilde{\Phi}}{\partial r} \Phi_{0}
$$

which becomes:

$$
\frac{\partial \Phi_{1}}{\partial r}=\frac{T_{e}}{e \Phi_{0}}\left[\frac{\partial \Phi_{0}}{\partial r}-\frac{\Phi_{0}}{L_{\tilde{\Phi}}}\right] \tilde{n}_{e}
$$

The formulation of Eq.(65) allows the use of measured $T_{e}$ gradients, while the formulation of Eq.(67) allows the use of experimental $\Phi_{0}$ and $v_{\phi}$, via the constraint

$$
\frac{\partial \Phi_{0}}{\partial r}=B_{\phi} v_{\theta j}-B_{\theta} v_{\phi j}
$$

Experimental measurements of the plasma potential on ISX-B ${ }^{|64|}$ have reported values of $\Phi_{o} \sim T_{e} / e$ in a plasma with intense NBI. Both forms suffer from the need to evaluate $L_{\tilde{x}}$ quantities approximately. Since the $\Phi_{0}$ data are non-existent for the TFTR experiments, we will base our calculations on Eq.(65) and discuss how the results would be affected if we had used Eq.(67).

The contribution of the potential to $\tilde{\Omega}_{\phi j}$, Eq.(63), can be formally written as $\tilde{\Omega}_{\phi j(\Phi)}=\mathcal{V}_{e(c, s)} \tilde{n}_{e(c, s)}$ and specifically as

$$
\tilde{\Omega}_{\phi j(\Phi)}=\frac{1}{B_{\theta}} \frac{T_{e}}{e} \frac{\tilde{n}_{e(c, s)}}{v_{\phi j}}\left(\frac{1}{L_{T_{e}}}+\frac{1}{L_{\tilde{n}_{e(c, \theta)}}}\right) \equiv \mathcal{V}_{e}^{*}\left(\frac{a}{L_{T_{e}}}+\frac{a}{L_{\tilde{n}_{e(c, s)}}}\right) \tilde{n}_{e(c, s)}
$$

when Eq.(65) is used to evaluate $\partial \Phi_{1} / \partial r$, or as

$$
\tilde{\Omega}_{\phi j(\Phi)}=\left(1-\frac{v_{\theta j}}{\beta v_{\phi j}}-\frac{\Phi_{0}}{B_{\theta} L_{\tilde{\Phi}} v_{\phi j}}\right) \frac{T_{e}}{e \Phi_{0}} \tilde{n}_{e}
$$

when Eq.(67) is used instead.

The contributions of inertia and viscosity terms are:

$$
\tilde{\Omega}_{\phi j\left(g_{r}\right)}+\tilde{\Omega}_{\phi j\left(\Pi_{r}^{o}\right)} \equiv U_{\jmath c} \cos \theta+U_{\jmath s} \sin \theta
$$

Summing all the terms that contribute to the poloidally varying part of $\Omega_{\phi j}$, one obtains:

$$
\tilde{\Omega}_{\phi j}(r, \theta)=\tilde{\Omega}_{\phi j c}(r) \cos \theta+\tilde{\Omega}_{\phi, \mu}(r) \sin \theta
$$


with:

$$
\begin{aligned}
& \tilde{\Omega}_{\phi j c}(r)=-\frac{v_{\theta j}}{v_{\phi j} \beta}\left(2 \epsilon+\tilde{n}_{j c}\right)+\mathcal{V}_{j o} \tilde{n}_{j c}+\mathcal{V}_{e c} \tilde{n}_{e c}+U_{j c} \\
& \tilde{\Omega}_{\phi j s}(r)=-\frac{v_{\theta j}}{v_{\phi j}} \frac{\tilde{n}_{j s}}{\beta}+\mathcal{V}_{j s} \tilde{n}_{j s}+\mathcal{V}_{e s} \tilde{n}_{e s}+U_{j s}
\end{aligned}
$$

The magnitude of the $\tilde{\Omega}_{\phi j}$ is determined by the magnitudes of $\tilde{n}_{j}$ and $v_{\theta j}$, which one must now proceed to evaluate.

\subsection{Poloidal Density Asymmetries and Poloidal Velocity}

The steady state poloidal projection of the momentum equation, for ion species $j$, is:

$$
g_{\theta j}+\frac{\partial}{\partial l_{\theta}} p_{j}+e_{j} n_{j} \frac{\partial \Phi}{\partial l_{\theta}}+e_{j} n_{j} v_{r j} B_{\phi}-\mathcal{M}_{\theta j}-R_{\theta j}+\Pi_{\theta j}+m_{j} v_{\theta j} \mathcal{S}_{j}=0
$$

where the inertia term can be written:

$$
\begin{aligned}
g_{\theta j} & =m_{j} n_{j}\left[-\frac{v_{\phi j}^{2}}{R} \frac{\partial R}{\partial l_{\theta}}+\frac{1}{2} \frac{\partial v_{\sigma_{j}}^{\prime \prime}}{\partial l_{e}}+\frac{v_{r j}}{r} \frac{\partial\left(r v_{\theta j}\right)}{\partial r}\right]= \\
& \left.=m_{j} n_{j}\left[v_{\phi j}^{2} \frac{\sin \theta}{R}+v_{\theta j}^{2}\left(\frac{\sin \theta}{R}-\frac{\vartheta_{j}}{r}\right)\right]+\frac{v_{r j}}{r} \frac{\partial\left(r v_{\theta j}\right)}{\partial r}\right]
\end{aligned}
$$

The flux surface average of $(\mathrm{Eq} .(75)) /\left(n_{j} \mathrm{~h}\right)$ is:

$$
\begin{aligned}
& m_{j} R_{0}<\Omega_{\phi j}^{2} \sin \theta>+\frac{m_{j}}{r}<\frac{v_{r j}}{h} \frac{\partial\left(r v_{\theta j}\right)}{\partial r}>+<\frac{e_{j} v_{r j} B_{\phi}}{h}> \\
& -<\frac{R_{\theta j}}{h n_{j}}>+<\frac{\Pi_{\theta j}}{h n_{j}}>-<\frac{\mathcal{M}_{\theta j}}{h n_{j}}>+<\frac{m_{j} v_{\theta j} \mathcal{S}_{j}}{h n_{j}}>=0
\end{aligned}
$$

Subtracting Eq.( 77 ) from (Eq. 75$) /\left(n_{j}\right)$, one obtains an equation that describes the poloidal variations of the density, as a result of the equilibrium between the pressure, electric field, centrifugal and viscous forces:

$$
\frac{1}{n_{j}} \frac{\partial n_{j}}{\partial \theta}+Z_{j} \frac{T_{e}}{T_{j}} \frac{1}{n_{e}} \frac{\partial n_{e}}{\partial \theta}+\frac{m_{j}\left(v_{\phi j}^{2}+v_{\theta j}^{2}\right)}{T_{j}} \epsilon \sin \theta+\frac{r}{T_{j}}\left(\frac{\Pi_{\theta j}}{n_{j}}-<\frac{\Pi_{\theta j}}{h n_{j}}>\right)+\mathcal{H}_{j}=0
$$

with

$$
\begin{aligned}
\mathcal{H}_{j} & \simeq-m_{j} \frac{\vartheta_{\theta j}^{2}}{T_{j}} \vartheta_{j}+\frac{r}{T_{j}}\left[e_{j} v_{r j} B_{\phi} \tilde{v}_{r j}-\frac{\mathcal{M}_{\theta j}}{n_{j}}-\left(\frac{R_{\theta j}}{n_{j}}-<\frac{R_{\theta j}}{h n_{j}}>\right)\right] \\
& +\frac{m_{j}}{T_{j}}\left[v_{\theta j} \frac{\partial\left(r v_{r j}\right)}{\partial r}-<\frac{v_{\theta j}}{h} \frac{\partial\left(r v_{r j}\right)}{\partial r}\right]+\frac{r}{T_{j}}\left(\frac{\Pi_{\theta j}}{n_{j}}-<\frac{\Pi_{\theta j}}{h n_{j}}>\right)_{\text {terms }}^{\text {ressdual }}
\end{aligned}
$$


We rewrite Eq.(78) as:

$$
\frac{1}{n_{j}} \frac{\partial n_{j}}{\partial \theta}+Z_{j} \frac{T_{e}}{T_{j}} \frac{1}{n_{e}} \frac{\partial n_{e}}{\partial \theta}+\mathcal{D}_{j}=0
$$

For a two ion species plasma ( $1=$ main ions; $I=$ light impurity) the system of Eqs. $(80)_{j=i, I}$ can be solved in terms of $\tilde{n}_{j}$. The presence of the metal impurities, which would affect the other ions through the potential and collision terms, can be neglected since we are considering discharges with $Z_{m e t} \equiv Z_{M}^{2} n_{M} / n_{e}=0.1-0.6(\mathrm{M}=$ metal impurity). The poloidal variation of the impurity density, in the case of $m_{I}=m_{i} Z_{I}$, $v_{\phi i} \sim v_{\phi I}$ and $T_{i} \sim T_{I}$ is described by:

$$
\frac{1}{n_{I}} \frac{\partial n_{I}}{\partial \theta}=\left[-\mathcal{D}_{I}\left(1+Z_{i}^{2} \frac{n_{i}}{n_{e}} \frac{T_{e}}{T_{I}}\right)+\mathcal{D}_{i} Z_{i} Z_{I} \frac{n_{i}}{n_{e}} \frac{T_{e}}{T_{I}}\right]\left(1+\frac{T_{e}}{T_{I}} Z_{e f f}\right)^{-1}
$$

For the main ions:

$$
\frac{1}{n_{i}} \frac{\partial n_{i}}{\partial \theta}=-\left[\mathcal{D}_{i}+Z_{i} Z_{I} \frac{n_{I}}{n_{e}} \frac{T_{e}}{T_{i}}\left(\frac{1}{n_{I}} \frac{\partial n_{I}}{\partial \theta}\right)\right]\left(1+Z_{i} \frac{n_{i}}{n_{e}} \frac{T_{e}}{T_{i}}\right)^{-1}
$$

In the case of $\eta_{0 i} v_{\theta i} Z_{I} T_{e} /\left(n_{e} T_{i}\right)<\eta_{0 I} v_{\theta I} / n_{I}$ and $Z_{i}=1$, which is the case for TFTR, the $\mathcal{D}_{i}$ term can be neglected and Eq.(81) becomes independent of $\tilde{n}_{i}$ and $v_{\theta i}$. One derives an expression for the impurity (carbon) density poloidal variation:

$$
\begin{aligned}
\frac{1}{n_{I}} \frac{\partial n_{I}}{\partial \theta} & =-\tilde{n}_{I c} \sin \theta+\tilde{n}_{I s} \cos \theta= \\
& -\left\{m_{I} v_{\phi I}^{2}-\frac{13}{2} \frac{\beta}{\omega_{c I}} \frac{\partial v_{\phi}}{\partial r}\left[T_{I}-\left(Z_{I}-1\right) \frac{n_{i}}{n_{e}} T_{e}\right]\right\}\left(T_{I}+T_{e} Z_{e f f}\right)^{-1} \epsilon \sin \theta \\
& +\left\{-\frac{\eta_{O I} v_{\theta I}}{r p_{I}} \frac{2}{3} \tilde{n}_{I s} \sin \theta-\frac{\eta_{O I} v_{\theta I}}{r p_{I}}\left(\epsilon+\frac{2}{3} \tilde{n}_{I c}\right) \cos \theta\right. \\
& \left.+\frac{\eta_{O I}}{\epsilon p_{I}} \beta a_{I s} \tilde{n}_{I c} \cos \theta+\frac{\eta_{0 I}}{\epsilon p_{I}} \beta \Omega_{\phi I} U_{I c} \cos \theta\right\} \frac{T_{I}+T_{e} n_{i} / n_{e}}{T_{I}+T_{e} Z_{e f f}} \equiv \\
& -E_{I} \sin \theta-C_{I} \tilde{n}_{I s} \sin \theta-C_{I}\left(\frac{3}{2} \epsilon+\tilde{n}_{I c}\right) \cos \theta+D_{I} \tilde{n}_{I c} \cos \theta+F_{I} \cos \theta
\end{aligned}
$$

The terms from $\Pi_{\theta I}$ that have been neglected in Eq.(83) are at least a factor of $\epsilon$ smaller than the terms that have been included. $\tilde{n}_{e} \sim \tilde{n}_{I} Z_{I} n_{I} / n_{e}$ has been assumed, anticipating $\tilde{n}_{i} \ll \tilde{n}_{I}$.

The term $E_{I}$ represents the centrifugal force and a contribution from the gyroviscous tensor; $C_{I}, D_{I}$ and $F_{I}$ are contributions from the parallel viscous tensor. 
The following definitions have also been used:

$$
\begin{aligned}
A_{j} & \equiv A_{j_{\mathrm{s}}} \sin \theta+A_{j_{\mathrm{a}}} \cos \theta \\
& \equiv a_{j_{\mathrm{s}}} \tilde{n}_{j \mathrm{c}} \sin \theta+a_{j_{\mathrm{a}}} \tilde{n}_{j_{\mathrm{s}}} \cos \theta+\Omega_{\phi j} U_{j \mathrm{a}} \sin \theta-\Omega_{\phi j} U_{j_{\mathrm{s}}} \cos \theta
\end{aligned}
$$

with

$$
\begin{aligned}
a_{j c} & =-\left[\frac{T_{j}}{e Z_{j} L_{\tilde{n}_{j}}}+\frac{T_{e}}{e} \frac{Z_{I} n_{I}}{n_{e}}\left(\frac{1}{L_{T_{e}}}+\frac{1}{L_{\tilde{n}_{I}}}\right)\right] \frac{1}{B_{\theta} R} \\
a_{j s} & =\left[\frac{T_{j}}{e Z_{j} L_{\tilde{n}_{j c}}}+\frac{T_{e}}{e} \frac{Z_{I} n_{I}}{n_{e}}\left(\frac{1}{L_{T_{e}}}+\frac{1}{L_{\tilde{n}_{I_{c}}}}\right)\right] \frac{1}{B_{\theta} R}
\end{aligned}
$$

Equation (83) can be solved for the poloidal variations of the density:

$$
\tilde{n}_{I c}=E_{I}+C_{I} \tilde{n}_{I s}
$$

and

$$
\tilde{n}_{I s}=\frac{D_{I} E_{I}-C_{I}\left(\frac{3}{2} \epsilon+E_{I}\right)+F_{I}}{1+C_{I}\left(C_{I}-D_{I}\right)}
$$

The $\tilde{n}_{I}$ 's are functions of $v_{\theta I}$, which must be determined by solving the FSA poloidal momentum balance equation, Eq.(77). To this end, one can write: < $\Omega_{\phi j}^{2} \sin \theta>\simeq\left(\Omega_{\phi j o} \Omega_{\phi j s}\right)$ and

$$
\Omega_{\phi I s} \simeq-\left(\frac{v_{\theta I}}{\beta R}+a_{I_{c}}\right) \tilde{n}_{I s}+\Omega_{\phi I} U_{I s}
$$

Substituting this expression for $\Omega_{\phi I s}$ in Eq.(77) and noting that the friction term and the terms from the gyroviscous tensor can be neglected, we obtain a polynomial in $v_{\theta I}$ (third degree) that must be solved with a numerical method:

$$
\begin{aligned}
& -m_{I} \tilde{n}_{I s} v_{\phi I}\left(a_{I c}+\frac{v_{\theta I}}{\beta R}\right)+m_{I} v_{\phi I}^{2} \frac{U_{I s}}{R}+\frac{e}{n_{I}} \gamma_{I} \Gamma_{e} B_{\phi} \\
& +\frac{\eta_{U I} v_{\theta I}}{n_{I} R^{2}}\left[-\frac{3}{2}+\frac{\tilde{n}_{I s}}{\epsilon}-\frac{\tilde{n}_{I c}}{2 \epsilon}+\frac{1}{3 \epsilon^{2}}\left(\tilde{n}_{I c}^{2}+\tilde{n}_{I s}^{2}\right)\right] \\
& +\frac{n_{1 I I} \beta}{n_{I} r}\left[A_{I A}\left(\frac{3}{2}-\frac{\tilde{n}_{I s}}{\epsilon}-\frac{\tilde{n}_{I c}}{2 \epsilon}\right)+A_{I c}\left(\frac{\tilde{n}_{I s}}{2 \epsilon}-\frac{\tilde{n}_{I c}}{\epsilon}\right)\right]=0
\end{aligned}
$$

The $L_{x} \mathbf{s}$, with $x=\bar{n}, v_{\theta}, \bar{\Omega}$, etc., are evaluated numerically with successive iterations. 
The poloddally asymmetric component of the main (deuterium) lon density, $\tilde{n}_{i}$, can be determined from Eq.(82), which can be rewritten as:

$$
\begin{aligned}
\frac{1}{n_{i}} \frac{\partial n_{i}}{\partial \theta} & =-\tilde{n}_{i c} \sin \theta+\tilde{n}_{i s} \cos \theta= \\
& -\left(m_{i} v_{\phi i}^{2}-\frac{13}{2} \frac{\beta}{\omega_{c i}} \frac{\partial v_{\phi}}{\partial r} T_{i}-T_{e} Z_{I} \frac{n_{I}}{n_{e}} \frac{\tilde{n}_{I c}}{\epsilon}\right)\left(T_{i}+T_{e}^{\prime} \frac{n_{i}}{n_{\theta}}\right)^{-1} \epsilon \sin \theta \\
& -\left(\frac{\eta_{0 i} v_{\theta i}}{r n_{i}} \epsilon+T_{e} Z_{I} \frac{n_{I}}{n_{e}} \tilde{n}_{I_{s}}\right)\left(T_{i}+T_{e} \frac{n_{i}}{n_{a}}\right)^{-1} \cos \theta \\
& -\frac{\eta_{0 i} v_{\theta i}}{r n_{i}} \frac{2}{3}\left(\tilde{n}_{i c} \cos \theta+\tilde{n}_{i s} \sin \theta\right)\left(T_{i}+T_{e} \frac{n_{i}}{n_{a}}\right)^{-1} \\
& +\frac{\eta_{0 i}}{\epsilon n_{i}} \beta\left(-a_{i c} \tilde{n}_{i c} \cos \theta+a_{i s} \tilde{n}_{i s} \sin \theta\right)\left(T_{i}+T_{e} \frac{n_{i}}{n_{e}}\right)^{-1} \\
& +\frac{\eta_{0 i}}{\epsilon n_{i}} \beta \Omega_{\phi i}\left(U_{i c} \cos \theta+U_{i s} \sin \theta\right)\left(T_{i}+T_{e} \frac{n_{i}}{n_{e}}\right)^{-1} \equiv \\
& -E_{i} \sin \theta-C_{i} \cos \theta-G_{i}\left(\tilde{n}_{i s} \cos \theta+\tilde{n}_{i s} \sin \theta\right) \\
& +D_{c i} \tilde{n}_{i s} \cos \theta+D_{s i} \tilde{n}_{i s} \sin \theta+F_{\mathrm{ci}} \cos \theta+F_{s i} \sin \theta
\end{aligned}
$$

Eq.(91) can be solved in terms of the poloidal variations of the main ions density:

$$
\tilde{n}_{i c}=E_{i}+\left(G_{i}-D_{s i}\right) \tilde{n}_{i s}+F_{s i}
$$

and

$$
\tilde{n}_{i s}=\frac{E_{i}\left(D_{c i}-G_{i}\right)-C_{i}+F_{c i}}{1+\left(G_{i}-D_{c i}\right)\left(G_{i}-D_{s i}\right)}
$$

The pololdal velocity of the main ions, of which the $\tilde{n}_{(c, s) i}$ are functions, can be determined by solving the FSA poloidal momentum balance equation for the main ions:

$$
\begin{aligned}
& -m_{i} \tilde{n}_{i s} v_{\phi i}\left(a_{i c}+\frac{v_{\theta i}}{\beta R}\right)+m_{i} v_{\phi i}^{2} \frac{U_{i s}}{R}+\frac{e}{n_{i}} \gamma_{i} \Gamma_{I} B_{\phi} \\
& +\frac{\eta_{0 i} v_{\theta i}}{n_{i} R^{2}}\left[-\frac{3}{2}+\frac{\tilde{n}_{i s}}{\epsilon}-\frac{\tilde{n}_{i c}}{2 \epsilon}+\frac{1}{3 \epsilon^{2}}\left(\tilde{n}_{i c}^{2}+\tilde{n}_{i s}^{2}\right)\right] \\
& +\frac{\eta_{0 i} \beta}{n_{i} r}\left[A_{i s}\left(\frac{3}{2}-\frac{\tilde{n}_{i s}}{\epsilon}-\frac{\tilde{n}_{i c}}{2 \epsilon}\right)+A_{i c}\left(\frac{\tilde{n}_{i s}}{2 \epsilon}-\frac{\tilde{n}_{i c}}{\epsilon}\right)\right] \\
& +\frac{\beta}{4} \frac{p_{1}}{\omega_{c s}}\left(-13 \frac{\partial \Omega_{\phi i s}}{\partial r}+3 \frac{\tilde{n}_{i s}}{\epsilon} \frac{\partial \Omega_{\phi i s}}{\partial r}-3 \frac{\tilde{n}_{i s}}{\epsilon} \frac{\partial \Omega_{\phi i c}}{\partial r}\right)=0
\end{aligned}
$$




\section{Anomalous effects}

The fluid equations used so far in this report describe neoclassical transport. It is well-known that, in general, there are several disagreements between experimental results and neoclassical predictions of ion transport (e,g. $\left.{ }^{(65)}\right)$.

Values of the radial electron particle flux and the radial ion heat flux interpretted from the experimental data in the TFTR experiments are much larger than the neoclassical prediction. We introduce anomalous effects into our calculations by using measured values of electron particle flux in our equations for $\tilde{n}_{j}$ and $v_{\theta j}$. However anomalous forces which produce these anomalous fluxes possibly also should be included in our poloidal momentum balance equations. We represent these anomalous forces by introducing a phenomenological factor which reduces the magnitude of the experimental fluxes that enter our calculation.

\subsection{Radial velocities}

The computation of the $\tilde{n}_{j}, \tilde{\Omega}_{j}$ and $v_{\theta j}$ radial profles requires $v_{r j}(r)$ as input. The radial electron flux can be evaluated from experimental data, as outlined in Section 2.2. Since the impurity density profile was not measured in TFTR, the radial ion fluxes have not been determined in this work. Nevertheless, the possible range of variability of $v_{r j}$ is defined by the ambipolarity condition:

$$
n_{e} v_{r e}=n_{i} v_{r i} Z_{i}+n_{I} v_{r I} Z_{I}
$$

and by the following observations.

The analysis of the plasma parameters has been restricted to the steady state phase of the discharge. During the last $600 \mathrm{~ms}$ of NBI, the plasma parameter measurements, including the $Z_{\text {eff }}$ measurement, appeared stationary. The impurity source is limited to a few cms near the plasma edge. According to the continuity equation, a steady state density and the absence of sources/sinks imply a vanishing radial particle flux. 
We can estimate an upper limit for the impurity flux magnitude related to possible variations of the impurity density which can occur during the steady state phase of the discharge and which are not clearly observable. The error affecting the total impurity particles $\Delta N_{I}$, where $N_{I}=\int_{V_{p}} n_{I} d V_{p}\left(V_{p}=\right.$ plasma volume), can be written in terms of $Z_{e f f}, N_{e}$ and their error bars. The impurity flux associated with such a variation of $N_{I}$ is: $(2 \pi)^{2} r R \Gamma_{I}=-\Delta N_{I} / \Delta t$, where $\Delta t$ is the time length of the steady state phase of the discharge. The ratio between the impurity and electron radial current is then:

$$
\frac{Z_{I} \Gamma_{I}}{\Gamma_{e}}=\frac{Z_{I} \Gamma_{I} \tau_{e}}{N_{e}}=\frac{\tau_{e}}{\Delta t}\left(\frac{\Delta N_{e}}{N_{e}} \frac{Z_{e f f}-1}{Z_{I}-1}+\frac{\Delta Z_{e f f}}{Z_{I}-1}\right)
$$

The particle confinement time, $\tau_{e}$, was in the range [0.2-0.4] $\mathrm{s}$ over most of the plasma region and it was $\simeq 0.3 s$ at $\mathrm{r} / \mathrm{a}=0.9$. With $Z_{\text {eff }}=3, \Delta N_{e} / N_{e}=0.1$ and $\Delta Z_{\text {eff }} / Z_{\text {eff }}=0.2^{|47|}$, values typical of TFTR, we obtain:

$$
Z_{I} \frac{\Gamma_{I}(a)}{\Gamma_{e}(a)}<0.1
$$

\subsection{Anomalous Forces}

The transport equations used to investigate fluctuation-induced fluxes include a number of additional terms rising from the time-averaging of the fluctuating plasma parameters. In the presence of electrostatic and magnetic fluctuations driven transport, the poloidal momentum balance equation becomes: (66), (67)

$$
\frac{\partial \bar{p}_{j}}{\partial l_{\theta}}+\bar{\Pi}_{\theta j}-e_{j} \bar{n}_{j} \bar{E}_{\theta}-e_{j} \ll \tilde{n}_{j} \tilde{E}_{\theta} \gg+\ll \tilde{J}_{\| j} \ddot{B}_{r} \gg+e_{j} B_{\phi} \bar{\Gamma}_{j}=0
$$

where $\bar{x}$ and $\tilde{x}$ indicate the time-averaged and fluctuating component of the plasma parameter $x$ in Eq.(98). The collision and momentum input frequencies were assumed to be smaller than the fluctuation frequency.

In case of entirely fluctuation driven radial particle fluxes, the first three terms of Eq.(98) can be neglected with respect to the remaining terms and the radial particle flux is given by $(65)$ :

$$
\tilde{\Gamma}_{j}=\ll \tilde{n}_{j} \tilde{E}_{\theta} \gg / B_{\phi}-\ll \tilde{J}_{\| j} \tilde{b}_{r} \gg / e_{j}
$$


Experimental evidence suggests that fluctuation characteristics (amplitude, parameter dependence, frequency, wave length, etc.) have similar parametric dependence in all toroidal fusion devices ${ }^{[65]}$, and this suggests that the electrostatic fluctuation term, in Eq.(98) could be also an important term for the TFTR plasma.

We include the term $\Gamma_{j}^{(\sim)}$ in the FSA poloidal momentum equation, Eq.(77), as a phenomenological representation of anomalous forces

$$
m_{j} v_{\phi j o} \Omega_{\phi j s}+Z_{j} \frac{\Gamma_{j} B_{\phi o}}{n_{j o}}-Z_{j} \frac{\Gamma_{j}^{(\sim)} B_{\phi o}}{n_{j o}}+<\frac{\Pi_{\theta j}}{h n_{j}}>\simeq 0
$$

We can define:

$$
\gamma_{j}^{\prime} \equiv Z_{j} \Gamma_{j} / \Gamma_{e} \quad \text { and } \quad \gamma_{j}^{n} \equiv \Gamma_{j}^{(\sim)} / \Gamma_{j}
$$

and rewrite:

$$
Z_{j}\left(\Gamma_{j}-\Gamma_{j}^{(\sim)}\right)=\gamma_{j}^{\prime} \Gamma_{e}\left(1-\gamma_{j}^{n}\right) \equiv \gamma_{j} \Gamma_{e}
$$

In the following numerical evaluation of $\tilde{n}_{j}$ and $\tilde{\Omega}_{\phi j}$, we consider values of $\gamma_{j}^{\prime}$ and $\gamma_{j}^{n}$ such that: $\left|\gamma_{I}^{\prime}\right| \leq 0.1$, according to Eq.(97) and related discussion; $\gamma_{i}^{\prime}+\gamma_{I}^{\prime}=1$, by ambipolarity, and $\gamma_{j}^{n}$ is a parameter assumed to vary in the range [0 - 1]. The limiting values $\gamma_{j}^{n}=0$ and $\gamma_{j}^{n}=1$ correspond, respectively, to the cases where the FSA of the poloidal projection of the anomalous force is negligible with respect to the particle flux, or it is its main driving term. With these values of $\gamma_{j}^{\prime}$ and $\gamma_{j}^{n}$ the ranges of variability of the $\gamma_{j}$ factors are: $\gamma_{i}=[0,1]$ and $\gamma_{I}=[0,0.1]$.

The anomalous term $\Gamma_{j}^{(\sim)}$ is not likely to affect the magnitude of $\tilde{n}_{j}$ directly through the poloidal momentum balance. However, the anomalous force will affect the magnitude of $\Pi_{\theta j}$ and $v_{\theta j}$ directly, and hence will affect $\tilde{n_{j}}$ indirectly via $\tilde{n_{j}}=\tilde{n_{j}}\left(v_{\theta j}\right)$.

\section{FORMULATIONS OF THE GYROVISCOUS TORQUE}

As discussed in Section 311, alm ance of information about the concentrations and poloidal asymmetries of the vanous ion species, it has been necessary to make 
assumptions about poloidal asymmetries for the different species. We are now able to evaluate poloidal asymmetries, albeit approximately. Thus, we reformulate the gyroviscous torque to include explicitly the poloidal asymmetries of the main ions (deuterium) and dominant impurity (carbon) in TFTR.

\subsection{Relation of poloidal asymmetries to gyroviscous torque}

The gyroviscous torque of Eq.(13) can be written

$$
<R \hat{\phi} \cdot \nabla \cdot \Pi_{j}^{3,4}>=-\frac{R_{0}}{r} \frac{\partial}{\partial r}\left(\eta_{4 j 0} v_{\phi j 0} \Xi_{j}\right)
$$

where

$$
\Xi_{j} \equiv \frac{\tilde{\Omega}_{\phi j s}\left(4 \epsilon+\tilde{n}_{j c}\right)-\tilde{n}_{j s} \tilde{\Omega}_{\phi j c}}{2}=\frac{1}{2} \epsilon^{2} \tilde{\Theta}_{j}
$$

The gyroviscous model that was used to analyze the experimental data was based on Eq.(16), which leads to an expression for the total torque summed over (ion) species

$$
\sum_{j}<R \vec{\phi} \cdot \nabla \cdot \Pi_{j}^{3,4}>=-\sum_{j} \frac{\tilde{\Theta}_{j}}{2} \frac{r}{R_{0}} \frac{\partial}{\partial r}\left(\eta_{4 j 0} v_{\phi j 0}\right)
$$

which, upon defining

$$
G_{j} \equiv-\frac{r}{\eta_{4 j 0} v_{\phi j}} \frac{\partial\left(\eta_{4 j} v_{\phi j}\right)}{\partial r}
$$

and assuming $v_{\phi j}$ independent of ion species, can be written

$$
\begin{aligned}
\sum_{j} & <R \hat{\phi} \cdot \nabla \cdot \Pi_{j}^{3,4}>=\sum_{j} \frac{\tilde{\Theta}_{j} G_{j}}{2} \frac{v_{\phi}}{R} \frac{m_{j} n_{j} T_{j}}{e B Z_{j}} \\
& =\frac{m_{D} n_{e} v_{\phi} T_{I}}{2 e B R} \sum_{j} \frac{n_{j 0}}{n_{e}} \tilde{\Theta}, G_{j} \equiv \frac{m_{D} n_{e} v_{\phi} T_{I}}{2 e B R}\left(\frac{\Theta G}{Z}\right)_{e f f}
\end{aligned}
$$

when $n_{j} \simeq m_{D} Z_{j}$ is used.

Two models for, in effect, estimating the quantity $(\Theta G / Z)_{\text {eff }}$ were discussed in Section 3.1.1. It was found in Section 5 that $(\bar{\Theta}=1.5, G=1, Z=\bar{Z})$ and $(\tilde{\Theta}=0.4$, $\left.G=1, Z=Z_{\text {eff }}\right)$ models could both reproduce the experimental data on $v_{\phi}(0)$ and $\tau_{\phi}$. Both of these models correspond to $(\Theta G / Z)_{\text {eff }} \sim O(0.1)$. Thus, in order to check 
the reasonableness of the values of $(\Theta G / Z)_{\text {eff }}$ chosen to fit the experimental data, we will calculate

$$
\left(\frac{\Theta G}{Z}\right)_{e f f} \equiv G_{i} \tilde{\Theta}_{i} \frac{n_{i}}{n_{e}}+G_{I} \tilde{\Theta}_{I} \frac{n_{I}}{n_{e}}
$$

Note that the contribution to $(\Theta G / Z)_{\text {eff }}$ from the metallic impurities is not being treated explicitly:

Charge neutrality and the definition of $Z_{\text {eff }}$ lead to

$$
\frac{n_{i}}{n_{e}}=\frac{Z_{I}-Z_{\text {eff }}}{Z_{I}-1} \quad \frac{n_{I}}{n_{e}}=\frac{Z_{\text {eff }}-1}{Z_{I}\left(Z_{I}-1\right)}
$$

\subsection{Different formulations of the gyroviscous torque flow}

The experimental torque flow is constructed by integrating the NBI torque density input over the volume bounded by radius $r$,

$$
K_{\phi}^{e x}(r)=(2 \pi)^{2} R \int_{0}^{r}<R \mathcal{M}_{\phi}>r^{\prime} d r^{\prime}
$$

when acceleration and convective terms can be neglected. $\mathcal{M}_{\phi}$ is the toroidal component of the NBI momentum density input rate and it has been assumed that the torque flow vanishes at $r=0$. The corresponding theoretical quantity is obtained by replacing $\left\langle R \mathcal{M}_{\phi}>\right.$ with $\sum_{j}<R \hat{\phi} \cdot \nabla \cdot \overrightarrow{\vec{\Pi}}_{j}>$. For the gyroviscous torque of Eq.(103)

$$
\begin{aligned}
K_{\phi}^{G V}(r) & =-(2 \pi R)^{2} \sum_{j} \int_{0}^{r} \frac{\partial}{\partial r^{\prime}}\left(\eta_{4 j} v_{\phi j} \Xi_{j}\right) d r^{\prime} \\
& =-(2 \pi R)^{2} \sum_{j} \int_{0}^{r} \eta_{4 j} v_{\phi j} \frac{\Xi_{j}}{\left(r^{\prime}\right)^{2}}\left(\frac{r^{\prime}}{\eta_{4 j} v_{\phi j} \Xi_{j}} \frac{\partial\left(\eta_{4 j} v_{\phi j} \Xi_{j}\right)}{\partial r^{\prime}}\right) r^{\prime} d r^{\prime} \\
& =(2 \pi R)^{2} \sum_{j} \int_{0}^{r} \eta_{4 j} v_{\phi j} \frac{\Xi_{j}}{\left(r^{\prime}\right)^{2}} G_{j} r^{\prime} d r^{\prime}
\end{aligned}
$$

where

$$
G_{j} \equiv-\frac{r^{\prime}}{\eta_{4 j} v_{\phi j} \Xi_{j}} \frac{\partial\left(\eta_{4 j} v_{\phi j} \Xi_{j}\right)}{\partial r^{\prime}}=-\frac{r^{\prime}}{\eta_{4 j} v_{\phi j}} \frac{\partial\left(\eta_{4 j} v_{\phi j}\right)}{\partial r^{\prime}}-\frac{r^{\prime}}{\Xi_{j}} \frac{\partial \Xi_{j}}{\partial r^{\prime}} \equiv G_{\eta v},+G_{\Xi_{j}}
$$

The original formulation of the gyroviscous torque flow was based upon making the separation of variables, or weak-radial-dependence of poloidal asymmetries, 
assumption in the evaluation of the radial profile factor $G_{j}$, which is equivalent to setting the $G_{\Xi_{j}}$ term to zero in Eq.(112). This leads to

$$
\begin{aligned}
K_{\phi}^{g v *} & =(2 \pi R)^{2} \sum_{j} \int_{0}^{r} \eta_{4 j} v_{\phi j} \frac{\Xi_{j}}{\left(r^{\prime}\right)^{2}} G_{\eta v_{j}} r^{\prime} d r^{\prime} \\
& =(2 \pi)^{2} \sum_{j} \int_{0}^{r} \eta_{4 j} v_{\phi j} \frac{\tilde{\Theta}_{j}}{2} G_{\eta v_{j}} r^{\prime} d r^{\prime}
\end{aligned}
$$

Making the further approximation that an effective value of $\Xi_{j} / \epsilon^{2}$ can be defined and removed from the integral leads to Eq.(20) and to

$$
K_{\phi}^{g v}=(2 \pi)^{2} \sum_{j} \frac{\Xi_{j}}{\epsilon^{2}} \int_{0}^{r} \eta_{4 j} v_{\phi j} G_{\eta v_{j}} r^{\prime} d r^{\prime}=(2 \pi)^{2} \sum_{j} \frac{\tilde{\Theta}_{j}}{2} \int_{0}^{r} \eta_{4 j} v_{\phi j} G_{\eta v_{j}} r^{\prime} d r^{\prime}
$$

which is the expression plotted in Fig. 11.

A third formulation of the gyroviscous torque flow, which avoids the separation of variables approximations, follows from integration of Eq.(111) over $0<r l<r$.

$$
\hat{K}_{\phi}^{g v}(r)=-(2 \pi R)^{2} \sum_{j}\left(\eta_{4 j} v_{\phi j} \Xi_{j}\right)_{\mid r}
$$

We have made use of plausibility arguments that the torque flux and the poloidal asymmetries must vanish as $r \rightarrow 0$ in deriving Equation (115). (We note that a similar development based on integration over $r<r^{\prime}<a$ leads to Eq.(115) with an additional term, $\hat{K}_{\phi}^{g v}(a)$, on the RHS. Thus, there is an ambiguity about Eq.(115) and how it is to be interpreted.)

$\hat{K}_{\phi}^{g v}$ is mathematically equivalent to $K_{\phi}^{g v *}$ when $G_{\eta v_{j}}$ is replaced by $\left(G_{\eta v_{j}}+G_{\Xi_{j}}\right)$ in Eq.(113).

\section{Calculated poloidal velocities and poloidal asym- metries}

The results of the calculations outlined in Section 7 are illustrated for one shot from the TFTR dedicated rotation experiment. Although the results vary slightly within the set of discharges, these results are quite representative of the whole experiment. 
For the impurities, Eq.(90) has one solution, $v_{\theta I}$, approximately given by:

$$
v_{\theta I} \simeq A_{I s} R \frac{\beta}{\epsilon}+\frac{2}{3} \frac{e \gamma_{I} \Gamma_{e}}{\eta_{0 I}} B_{\phi} R^{2}
$$

and plotted in Fig. 14, for $\gamma_{I}=0.0$ and 0.1 . The poloidal velocity is driven by the pressure radial gradient and by the radial flux, when present. In the case of $\gamma_{I}=0.1$, the first and third terms of Eq.(90) are the driving terms for $v_{\theta I}$ in the central and edge region, respectively. A similar result is obtained when $T_{e} /\left(e \Phi_{0}\right)$ varying from $\sim 1$ at the center to $\sim 0.1$ at the edge is used in Eq.(70).

These estimates of $v_{\theta I}$ are 1-2 orders of magnitude smaller than measurements of $v_{\theta I}$ in TFTR and in other machines $\{68\},(69),(70)$. Also, these small values of $v_{\theta I}$ imply a value of the parameter $T_{e} /\left(e \Phi_{0}\right) \sim 0.1$. This disagreement may be due to the fact that the heat flux, here neglected, plays an important role in determining the magnitude of $v_{\theta j}$, or to anomalous effects.

In order to investigate the effect of a larger poloidal velocity on the magnitude of $\tilde{n}_{j}$ and $\tilde{\Omega}_{\phi j}$, we introduce, as input to the calculation, a value of $v_{\theta I}$ obtained from a "trial" poloidal velocity profile, $v_{\theta *}$, shown in Fig. 14, and based on the experimental observation of $v_{\theta I}(r / a \sim 0.25)=1.210^{4} \mathrm{~m} / \mathrm{s}$ in TFTR and $v_{\theta I} \sim v_{\phi i} \sim O\left(10^{4}\right)$ at the edge of several other machines. We note that this constructed $v_{\theta *}$ is much flatter than other calculated or inferred (from $\Phi_{0}$ and $v_{\phi}$ measurements) velocity profiles and that this may overemphasize $v_{\theta}$-driven effects in the outer part of the plasma.

We find that in order to reproduce the $v_{\theta *}$ profile with our model, we would need to use $\gamma_{I} \sim O(1)$ or $T_{e} /\left(e \Phi_{0}\right)$ varying from $O(1)$ in the center to $O(0.1)$ at the edge. $\gamma_{I} \sim O(1)$ can only be interpretted as a phenomenological representation of anomalous forces, given the discussion in Section 8. These values of $T_{e} /\left(e \Phi_{0}\right)$ seem plausible based on the ISX-B measurements and associated calculations ${ }^{\mid 64)}$.

The corresponding poloidal asymmetries of the impurity density have been plotted in Fig. 15. The in-out asymmetry is essentially driven by the centrifugal force, that is: $\tilde{n}_{I c} \simeq \epsilon m_{I}\left(v_{\phi I}+v_{\theta I}\right)^{2}\left(T_{I}+T_{e} \frac{n_{I}}{n_{e}}\right)^{-1}$ while the up-down asymmetry, in this case, is 
given by:

$$
\tilde{n}_{I s} \simeq-\frac{r}{n_{I}} \frac{\Pi_{\theta I c}}{T_{I}+T_{e} n_{i} / n_{e}}=-\frac{\eta_{0 I}}{n_{I} R}\left(v_{\theta I}-R \frac{\beta}{\epsilon} a_{I s} \tilde{n}_{I c}\right)\left(T_{I}+T_{e} Z_{e f f}\right)^{-1}
$$

As already discussed, $\tilde{n}_{I c}<\epsilon$ because the carbon impurity ions are subsonic.

$\tilde{n}_{I s}$ is driven by the parallel viscous force and is negative over all the plasma region. The $\tilde{n}_{I s}\left(v_{\theta *}\right)$ profile has been calculated using $v_{\theta I}=v_{\theta *} ;$ its magnitude is $\sim 0.1 \epsilon$ and $\sim \epsilon$ at $\mathrm{r} / \mathrm{a}=0.5$ and $\mathrm{r} / \mathrm{a}=1$ respectively.

The radial profile of $v_{\theta i}$ is shown in Fig. 16 for three different values of $\Gamma_{i}\left(\gamma_{i}=\right.$ $\left.0.0,0.5,0.9 ; \gamma_{I}=0.1\right)$. The main ions poloidal velocity is positive and approximately given by:

$$
v_{\theta i} \simeq A_{i s} R \frac{\beta}{\epsilon}+\frac{2}{3} \frac{e \gamma_{i} \Gamma_{e}}{\eta_{0 i}} B_{\phi} R^{2}
$$

The first term is important only in the central region and for small $\Gamma_{i}$; otherwise the main ions poloidal velocity is essentially driven by the radial flux term.

The in-out asymmetry of the main ion density is coupled to the in-out impurity density asymmetry through the potential gradient, and it is approximately given by:

$$
\tilde{n}_{i c} \simeq E_{i}=-\epsilon\left[m_{i}\left(v_{\phi i}+v_{\theta i}\right)^{2}-\frac{13}{2} \frac{\beta}{\omega_{c i}} \frac{\partial v_{\phi}}{\partial r} T_{i}-T_{e} Z_{I} \frac{n_{I}}{n_{e}} \frac{\tilde{n}_{I c}}{\epsilon}\right]\left(T_{i}+T_{e} \frac{n_{i}}{n_{e}}\right)^{-1}
$$

The electric field and centrifugal forces are of the same order of magnitude and have opposite effects: the electric field tends to drive a negative in-out main ion density asymmetry in order to re-establish $\partial n_{e} / \partial \theta=0$.

The combined effects of viscous and electrostatic forces determine the magnitude of $\tilde{n}_{i s}$ :

$$
\begin{aligned}
\tilde{n}_{i s} \simeq-C_{i} & =-\left(\frac{r \Pi_{\theta i c}}{n_{i}}+T_{e} Z_{I} \frac{n_{I}}{n_{e}} \tilde{n}_{I s}\right)\left(T_{i}+T_{e} \frac{n_{i}}{n_{e}}\right)^{-1} \\
& =-\left(\frac{\eta_{0 i} v_{\theta i}}{r n_{i}} \epsilon+T_{e} Z_{I} \frac{n_{I}}{n_{e}} \tilde{n}_{I s}\right)\left(T_{i}+T_{e} \frac{n_{i}}{n_{e}}\right)^{-1}
\end{aligned}
$$

Radial profiles of $\tilde{n}_{\text {is }}$ are shown in Fig. 17 for different values of the ion radial flux term $\left(\gamma_{i}=0.0,0.5,0.9 ; \gamma_{I}=0.1\right)$. The relatively small values of $\tilde{n}_{j}$ s predicted by 
these calculations are consistent with the observation of up-down symmetric $Z_{e f f} n_{e}^{2}$ radial profile to within $10 \%$ measurement accuracy.

The $\tilde{\Omega}_{\phi j c}$ profile is essentially $\sim v_{\theta j} \epsilon /\left(\beta v_{\phi j}\right)$. Due to the relatively large $v_{\theta j}$, the in-out asymmetries, $\tilde{\Omega}_{\phi j c}$, are $O(\epsilon)$ and larger. Both up-down asymmetries, $\tilde{\Omega}_{\phi i s}$ and $\tilde{\Omega}_{\phi I s}$ are $<<\epsilon$ in the case $\gamma_{i}=\gamma_{I}=0.1$. When the calculation is performed using the "measured" $v_{\theta *}$ for $v_{\theta I}$, the relatively large $\tilde{n}_{I s}$ give rise to a $\tilde{\Omega}_{\phi I s}(r>a / 2)$ of $O(\epsilon)$ and larger.

$\Xi_{i}\left(\gamma_{i}=0.1\right)$ and $\Xi_{I}\left(\gamma_{I}=0.1\right)$ are positive and two orders of magnitude smaller than $\epsilon^{2}$ over most of the plasma region. $\Xi_{i}$ increases with $\gamma_{i}$ because of the increase of $\tilde{n}_{i}$ and $\tilde{\Omega}_{\phi i}$ with $\gamma_{i}, \Xi_{i}\left(\gamma_{i}=0.9\right)$ is one order of magnitude smaller than $\epsilon^{2}$ over most of the plasma region. The magnitude of $\Xi_{j}$ becomes comparable and even larger than $\epsilon^{2}$ at the plasma edge because of large values of $\tilde{\Omega}_{\phi j}$ in this region. When the calculation is performed using the "measured" $v_{\theta *}$ for $v_{\theta I}, \Xi_{I}$ is $O(0.1-1) \epsilon^{2}$ in the plasma interior and becomes $O\left(10 \epsilon^{2}\right)$ toward the plasma edge; the large edge value is due to the flat $v_{\theta *}$ profile and is probably unrealistic.

The $(\Theta G / Z)_{e f f}$ parameter, Eq.(108), has been evaluated for the case $\gamma_{i}=0.9, \gamma_{I}=$ 0.1 and for the case $\gamma_{i}=0.9$ and $v_{\theta I}=v_{\theta *}$. The results of the calculations are shown in Fig. 18. In the first case, we find $(\Theta G / Z)_{\text {eff }} \sim 0.1$ in the plasma center and $(\Theta G / Z)_{\text {eff }}$ increasing up to $\sim 10$ at the plasma edge. In the second case, the function $(\Theta G / Z)_{e f f}$ increases from $\sim 10^{-2}$ in the center up to $\sim 10$ at the edge. Large values at the edge are probably spurious.

In Section 5 it was found that using a radially-averaged value of $(\Theta G / Z)_{e f f} \sim 0.1$ led to predictions of momentum confinement times and central rotation velocities that agreed reasonably well with experiment. These calculations of the poloidal asymmetries support $(\Theta G / Z)_{e f f}$ of $\mathrm{O}(0.1)$ but suggest a radial dependence. The main ion asymmetries contribute significantly to the value of $(\Theta G / Z)_{e f f}$, which is different than previously thought $21 \mid$.

The magnitude of the gyroviscous torque flows $\hat{K}_{\phi}$ and $K_{\phi}^{g 1 *}$, given in Eqs. (115) 
and (113) are illustrated in Fig. 19 for the case $\gamma_{i}=0.9$ and $\gamma_{I}=0.1$. The experimental torque flow, $K_{\phi}^{e x}$, and the $K_{\phi}^{g v}$ profile result (evaluated for $\tilde{\Theta}=1.5$ and $\bar{Z}=Z_{\text {eff }}$ ) are also shown. The $K_{\phi}^{g v *}$ profile was evaluated taking into account the radial dependence of $(\Theta G / Z)_{e f f}$ evaluated from experimental $\eta_{4 j}$ and $v_{\phi j}$ profiles and calculated poloidal asymmetries. Both $K_{\phi}^{g v}$ and $K_{\phi}^{g v *}$ have similar magnitude and radial dependence (close to the magnitude and the profile of $K_{\phi}^{e x}$ ). We conclude that the difference in the formulation of $K_{\phi}^{g v}$. (i.e. $\tilde{\Theta}=\operatorname{const}(r)$ and $G_{\Xi_{j}}=0$ ) and $K_{\phi}^{g v *}$ (i.e. $\tilde{\Theta}(r)$ and $G_{\Xi_{j}}=0$ ) did not generate a considerable difference in the results. The magnitude of $K_{\phi}^{g v *}$ is a factor of two smaller than $K_{\phi}^{c x}$ over most of the plasma bulk. Thus, using poloidal asymmetry factors $\tilde{\Theta}_{j}$, or $\Xi_{j}$, evaluated from first-principle calculations of the $\tilde{n}_{j}$ and $\tilde{\Omega}_{\phi j}$, Eq.(113) for $K_{\phi}^{g v *}$ predicts the experimental torque flow to within a factor of two over most of the plasma.

For the case $\left|\Gamma_{j}^{(\sim)}\right| \leq\left|\Gamma_{j}\right|$, rather large values of $v_{\theta i}$ are obtained, and the dominant contribution to $K_{\phi}^{g v *}$ is from the deuterium ions. When $v_{\theta I}=v_{\theta *}$ is used, $n_{I s} \sim \tilde{\Omega_{I s}} \sim$ $O(\epsilon)$ and the dominant contribution to $K_{\phi}^{g v *}$ is from the carbon ions.

The $\hat{K}_{\phi}^{g v}$, which was evaluated from Eq.(115) using calculated $\Xi_{j}$ and $G_{\Xi_{j}}$ profiles, is negative and one order of magnitude smaller than $K_{\phi}^{e x}$. The difference between $\hat{K}_{\phi}^{g v}$ and $K_{\phi}^{g v *}$ is entirely in the different treatment of $G_{\Xi_{j}}$, which is neglected in $K_{\phi}^{g v *}$. We calculate $G_{\Xi_{j}}$ by solving for $\Xi_{j}$ at different radial locations and evaluating the radial gradient numerically. Figure 20 shows the radial profiles of $G_{\Xi_{i}}$ and $G_{\eta v_{i}}$ for the case $\gamma_{I}=0.1$ and $\gamma_{i}=0.9 ;$ they have similar magnitude and radial profiles but opposite signs. Indeed the $G_{\Xi_{i}}$ function is not negligible with respect to $G_{\eta v_{i}}$; actually $\left|G_{\Xi_{i}}\right|$ is larger than $G_{\eta v_{i}}$ and this explains why $\hat{K}_{\phi}^{g v}$ is negative. We reiterate that the interpretation of $\hat{K}_{\phi}^{g v}$ is ambiguous, as discussed in Section 9.2.

From Eqs. (111-115), it follow's that the two formulations of the total gyroviscous torque flows may be written

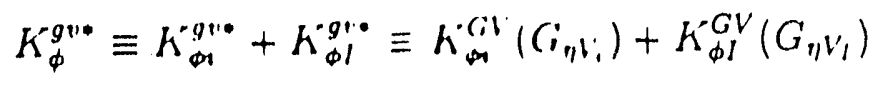


and

$$
\hat{K}_{\phi}^{g v} \equiv \hat{K}_{\phi i}^{g v}+\hat{K}_{\phi I}^{g v} \equiv\left[K_{\phi i}^{G V}\left(G_{\eta V_{i}}\right)+K_{\phi i}^{G V}\left(G_{\Xi_{i}}\right)\right]+\left[K_{\phi I}^{G V}\left(G_{\eta V_{I}}\right)+K_{\phi I}^{G V}\left(G_{\Xi_{I}}\right)\right]
$$

where $K_{\phi}^{G V}$ is given by Eq.(111).

The original gyroviscous formulation, $K_{\phi}^{g 1 *}$, which assumed $G_{\Xi_{j}}=0(\mathrm{j}=\mathrm{i}, \mathrm{I})$, agrees rather well with the experimental torque flow. However, based upon our model for calculating the poloidal asymmetry factor $\Xi_{j}$, we find $G_{\Xi_{j}} \neq 0$ and of comparable magnitude (and opposite sign) as $G_{\eta V_{j}}$, resulting in a near cancellation between $K_{\phi j}^{G V}\left(G_{\eta V_{j}}\right)$ and $K_{\phi j}^{G V}\left(G_{\Xi_{j}}\right)$ for each species. As a consequence of this near cancellation, we find that $\hat{K}_{\phi}^{g v}$ underpredicts the magnitude of the experimental torque flow by an order of magnitude or more.

We believe that the qualitative result, $G_{\Xi_{j}}<0$, is probably true independent of the calculational model for the poloidal asymmetries. However, the degree of cancellation between $\hat{K}_{\phi j}^{G V}\left(G_{\eta V_{j}}\right)$ and $\hat{K}_{\phi j}^{G V}\left(G_{\Xi_{j}}\right)$ is dependent on the calculational model for the poloidal asymmetry factor, $\Xi_{j}(r)$. For example, using spatially dependent values of the parameters $\gamma_{j}$, to allow a spatial variation of the anomalous forces which these parameters represent, could alter the $\Xi_{j}(r)$ profile. We are unable, based on available information, to carry out a meaningful study of any such variations in the calculational model.

In spite of the good agreement of $K_{\phi}^{g v}$ and $K_{\phi}^{g v *}$ with the experimental torque flow, one must conclude that the approximation (the separation-of-variables, $G_{\Xi_{j}}=0$ ), on which these two formulas are based, is not supported by our calculations of the radial dependence of the poloidal asymmetry factor $\Xi_{j}$. 


\section{Comparison with previous work}

\subsection{Parametric dependence of experimental $v_{\phi}(0), \tau_{\phi}$ and $\chi_{\phi}(r)$}

The question of how $v_{\phi}^{e x}(0)$ scales with the main machine parameters has been investigated since the earliest rotation experiments (PLT ${ }^{[1|,| 2 \mid}$, PDX $|3|$, ISX-B $|4|$, DIII

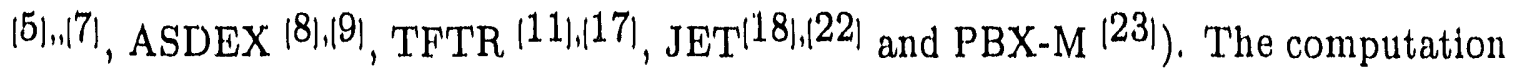
of $\tau_{\psi}^{e x}$, including profle effects, has been carried out extensively only in recent experiments (DIII, ASDEX, JET and TFTR), since it requires information on the radial profiles of $n_{e}$ and $v_{\phi}$.

The central rotation velocity was found: (a) to be $\propto T_{\phi} / \tilde{n}_{e}$ in PDX, and $\propto T_{\phi}^{\prime}$ $\left(\bar{n}_{e}=\right.$ const $)$ in PLT, DIII(divertor) and PBX-M ( $\bar{n}_{e}=$ const $) ;(\mathrm{b})$ to be $\propto\left(T_{\phi} / \bar{n}_{e}+\right.$ const) in TFTR and ASDEX; $(c)$ to saturate with increasing $T_{\phi} /\left[n_{c}\right]$ in JET and with increasing $T_{\phi}$ in ISX-B (with $\bar{n}_{e}=$ const) and DIII(limiter) (and $\bar{n}_{e}$ dependent on $P_{b}$ ). In these present TFTR experiments, we find $v_{\phi}(0) \sim T_{\phi} / n_{e}$.

$\tau_{\phi}^{e x}$, computed from measured profiles, was found: (a) to decrease with $T_{\phi}$ in limiter and divertor DIII discharges (even if for different reasons); (b) to depend essentially on density peaking in $\operatorname{ASDEX}_{i}(\mathrm{c})$ to deteriorate, modestly, with $P_{b}$ in the TFTR counter-NBI. In these present TFTR experiments, we find $\tau_{\phi}^{e x}$ decreases weakly with $T_{\phi} / n_{e}$.

The $I_{p}$ dependence found for $v_{\phi}(0)$ in the present TFTR discharges was absent in most of the other machines, with the exception of DIII, where $\tau_{\phi}^{e x}$ showed a linear increase with increasing $I_{p}$, which was attributed to $v_{\phi}$ profile broadening with $I_{p}$.

There are substantial differences among the different machines on how the momentum confinement in co- and counter-NBI compares: (a) the same magnitude of rotation velocity was obtained in PLT for the same magnitude of $T_{\phi} / \tilde{n}_{e}$; (b) a substantial improvement of momentum confinement, due to a simultaneous increase of $n_{e}$ and $v_{\phi}$, was observed in the ASDEX counter-NBI, relative to co-NBI $\left(\right.$ c) $v_{\phi}(0)$ 
was lower in the TFTR L-mode shots with counter NBI than in the co-NBI at the same magnitude of $T_{\phi} / \eta_{e}$. In this TFTR experiment, we find the rotation velocity is greater for co-NBI than for counter-NBI,

The trend towards larger momentum confinement time in larger machines is conflrmed by the present results.

\subsection{Comparison of theory with experiments for $v_{\phi}, \tau_{\phi}$ and $K_{\phi}(r)$}

Previous comparisons of theory and experiments have been most extensive for the gyroviscous theory. Measured and predicted magnitudes for $\tau_{\phi}$ and/or $v_{\phi}$ were found to be in reasonable agreement in ISX-B ${ }^{|25|}$, PLT $^{|25|}$, DIII ${ }^{|7|}$, JET ${ }^{|21|}$ and TFTR 11) L-modes. Observed parametric dependences could be accounted for in ISX-B, PLT, JET and some of the TFTR data. Disagreement between plasma parameter dependences predicted by the gyroviscous theory and observed in the experiments were reported in DIII and other TFTR data. The agreement between gyroviscous theory and experiment that we find for the present TFTR data is comparable to the agreement found in Refs. [[21]] and [[25]].

As discussed in Section 9.1, the different approximate models for summing the gyroviscous torque over species that have been employed in these past analyses correspond to choosing a value of the parameter $(\Theta G / Z)_{\text {eff }}$ defined by $\mathrm{Eq} .(108)$. The choice of $\Theta G=1$ and $Z=Z_{\text {eff }} \simeq 2.5$ in ISX-B, PLT and DIII corresponds to $\left.(\Theta G / Z)_{\text {cff }}\right) \simeq 0.4$. The choice of $\Theta G=1$ and $Z=\bar{Z} \simeq 1.5$ in JET correponds to $(\Theta G / Z)_{\text {aff }} \simeq 0.08$. We found that the choice $\Theta G=1.5$ and $Z=\hat{Z}=15$, corresponding to $(\Theta G / Z)_{e f f} \simeq 0.1$ fit the TFTR data quite well. The larger values of $\Theta G / Z)_{e f f}$ in ISX-B, PLT and DIII than in JET and TFTR would require that larger values of the poloidal asymmetry factor, $\tilde{\Theta}_{j}$, which in turn would require larger values of the poloidal density asymmemes. It is plausible that inertial and friction forces could drive larger density asymmetries for more collisional, more nearly sonic ions in 
ISX-B, PLT and DIII than in JET and TFTR, thus supporting largor values of $\Theta$ in the former, but we have not made these calculations.

A comparisen of the predictions of the 'smp' theory with momentum conflnement times in several tokamaks (ISX-B, TFTR, PLT, DITE and PBX), was reported in Ref.[29]. The magnitude of the predicted $\tau_{\phi}$ was found to agree with the measurements, but the limited number of discharges considered (one for each machine) and the lack of proflle information does not allow any conclusions about the parametric dependence and the local value of the 'smp' momentum flux. We would infer a much poorer agreement with experiment for the present data, based on our calculations of momentum diffusivities.

A previous comparison $|16|$ of $\chi_{\phi}^{\text {a.r }}$ and $\chi_{\phi}^{\eta_{i}}$ for the TFTR experiments in this report and for a subsequent series of L-mode shots found results similar to those reported In Section 5 for the ITG mode thenry.

\subsection{Density Poloidal Asymmetries}

Several theoretical analyses and experimental investigations pointed out that density poloidal asymmetries of $O(\epsilon)$ and larger could be observed (and have been) in speciflc plasma regimes. Refs. [71], [27] predicted that centrifugal force can drive in-out density poloidal asymmetry, and that $\tilde{n}_{j c} \sim \epsilon$ for sonic ' $y$ ' lon species. We arrive at the same conclusion, but $\tilde{n}_{j c}<\epsilon$ in these TFTR experiments because both main tons and carbon are subsonic.

Refs. [3], [72], [71], [27] and [73] concluded that up-down ion density asymmetries could be observed in the case of collisional ions. In fact, when the condition $\delta_{\theta i} \nu_{i i} Z_{I}^{2} / \omega_{t r i} \sim 1$ is satisfled, the friction term in Eq. (75) becomes the driving force of $\tilde{n}_{a j} \sim \epsilon$. This condition is not satisfied in these TFTR experiments where the friction force, in Eq. (75), is negligible with resprect to the parallel viscous force. In the case of the TFTR plasmas, it is the viscous force term which drives $\tilde{n}_{a j}$. 
Ref. [03] pointed out that asymmetric particle sources can drive $\tilde{n}_{j}$. In Section 7.1, we argued that this can also be the case for the TFTR plasmas only near the plasma edge.

In Ref. [74] it was found that momentum input can drive $\tilde{n}_{I s} \sim \epsilon$. In case of $\tilde{n}_{j}$ mainly driven by momentum input, one would obtain: $\frac{1}{n_{j}} \frac{\partial n_{j}}{\partial \theta} \propto \bar{M}_{\phi j} \propto Z_{j}^{2} \tilde{n}_{j}$. In particular, in the presence of sonic impuritles (high Z impurities) the in-out density asymmetry, driven by centrifugal force, scales as $Z_{j}$ and one obtains: $\tilde{n}_{j s} \propto \tilde{M}_{\phi j s} \propto$ $Z_{j}^{2} \tilde{n}_{j c} \propto Z_{j}^{3}$. The calculations of $\tilde{n}_{j s}$ in ISX-B and PLT $|74|$ were performed for tungsten $\left(Z_{W}=74\right)$ and titanium $\left(Z_{T_{i}}=22\right)$ and an input power in the range $[-2.4$ - 2.4] MW. Even taking into account the fact that in TFTR the input power can be higher, the momentum input would not drive any signiflcant up-down asymmetry of the carbon impurity density $\left(Z_{C}=6\right)$ in TFTR.

\subsection{Electrostatic potential asymmetry}

As shown in Eq. $(75), e_{j} \partial \Phi /(r \partial \theta)$ is a potential driving force for $\tilde{n}_{j}$, In addition $\tilde{\Phi}$ appears in the $\tilde{\Omega}_{\phi j}$ expression (Eq.(70)) and, if large enough, can determine its magnitude.

In Ref. [75], $\tilde{\Phi}$ was evaluated in case of perpendicular NBI and found to be of $O(\epsilon)$. This was the effect of a relatively large in-out density poloidal variation of the fast ions, a large fraction of which were trapped in the outer region of the tokamak by the "magnetic mirror force".

The presence of the fast ions is not accounted for in our calculation of poloidal variations. Nevertheless we can here discuss the effect of their presence upon $\tilde{\Phi}_{c}$ and therefore $\tilde{n}_{j c}$.

The contribution of an asymmetric fast ion density, $\tilde{n}_{f i}$, to $\tilde{\Phi}_{c}$ is given by:

$$
\tilde{\Phi}_{c(f i)} \frac{e \Phi_{o}}{T_{i}}=\tilde{n}_{f i} \frac{n_{f i}}{n_{e}}\left(\frac{T_{i}}{T_{e}}+Z_{e f f}-\frac{n_{f i}}{n_{e}}\right)^{-1}
$$


Using the following values, characteristic of TFTR: $n_{f i} / n_{e}=0.25, T_{i} / T_{e}=2.5$ and $Z_{\text {eff }}=3$, one obtains:

$$
\tilde{\Phi}_{a(f i)} \frac{e \Phi_{o}}{T_{i}} \simeq 0.05 \tilde{n}_{f i}
$$

Chang and Harvey ${ }^{175}$ ] evaluated $\tilde{n}_{f i}=0.5-0.6$ for quasi-perpendicular injection in DIII and PDX, and $\tilde{n}_{f i}=0.1$ for tangential injection in ISX-B. Using these values to estimate a possible range of variation of $\tilde{n}_{f i}$ in TFTP (whose NBI has a perpendicular component), one estimates: $0.005<\tilde{\Phi}_{\mathrm{c}(f i)} e \Phi_{o} / T_{i}<0.025$. In TFTR $\epsilon=0.3 \mathrm{r} / \mathrm{a}$. Therefore $\tilde{\Phi}_{c(f i)} \ll \epsilon$ when we make use of the ordering $e \Phi_{o} / T_{e} \sim 1$.

Chang and Hazeltine ${ }^{172 \mid}$ pointed out that $\tilde{n}_{j} Z_{j} n_{j} / n_{e} \sim \epsilon$ would lead to $\tilde{\Phi} \sim \epsilon$. This is consistent with our expression of $\tilde{\Phi}$. But in the shots analyzed in this work $\tilde{n}_{j} \ll \epsilon$ and therefore $\tilde{\Phi} \ll \epsilon$.

\subsection{Poloidal velocities}

$v_{\theta i}$ and $v_{\theta I}$ were calculated in Ref. [74] for collisional impurities and main ions in the plateau regime (ISX-B, PLT'), by solving the FSA parallel momentum balance equation. It was found that $v_{\theta I} \sim O\left(\beta v_{\phi}\right)>v_{\theta i}$, driven by momentum input and, to a lesser extent, by pressure gradients. This is consistent with the fact that $\eta_{0 i}>\eta_{0 I}$ and that $v_{\theta i} \eta_{0 i} \simeq v_{\theta I} \eta_{0 I}$. Analogously we solve the poloidal FSA polvidal balance equation and find $v_{\theta i} \sim O\left(\beta v_{\phi}\right)>v_{\theta I}$, consistent with $\eta_{0 i}<\eta_{0 I}$ and with the fact that both ions and impurities are in the banana regime.

\section{Summary}

A well-diagnosed rotation experiment has been carried out in TFTR with the purpose of obtaining measurements with the recently installed CHERS diagnostic of the radial profiles of $v_{\phi}$ and $T_{i}$ for a variety of plasma and machine parameter conditions. These measurements allowed the computation of the radial profiles of the torque flux and 
momentum diffusivities, quantities that previously were only known at the edge or unknown, as well as of global properties such as the momentum confinemınt time.

The data were analyzed with the 1D transport code SNAP. The discharges in this experiment showed characteristics intermediate between L-mode shots and Supershots. Rotation velocities, up to $5.510^{5} \mathrm{~m} / \mathrm{s}$, were found to be higher in low current, co-injection shots and in low B discharges. Experimental momentum confinement times, in the range $24-50 \mathrm{~ms}$, were found to decrease weakly with input torque per particle, to increase with plasma current and to be independent of $B$.

The momentum confinement properties $\left(\tau_{\phi}, v_{\phi}(0), K_{\phi}\right.$ and $\left.\chi_{\phi}\right)$, inferred from experimental data, were compared with the predictions of several neoclassical and anomalous theories. The gyroviscous theory, with the assumption of $O(1)$ poloidal asymmetry factors, $\tilde{\Theta}$, was shown to predict the magnitude and the parametric dependence of $\tau_{\phi}, v_{\phi}(0)$ and $K_{\phi}$. The perpendicular momentum flux caused by the presence of a plausible amount of cold ions was found to be negligible. Each of the anomalous theories considered (ITG, untrapped particles and stochastic magnetic perturbation modes) predicted torque flows with magnitudes, radial profiles and parametric dependences on plasma parameters different from those of the experimental torque flow.

Since the gyroviscous formalism was successful in predicting $\tau_{\phi}, v_{\phi}(0)$ and $K_{\phi}$, when an effective poloidal asymmetry factor of $\tilde{\Theta}=1.5$ was used, a detailed calculation of $\tilde{n}_{j}$ and $\tilde{\Omega}_{\phi j}$ was performed. Poloidal density asymmetries smaller than $\epsilon$ were calculated for both ions and impurities. These calculated poloidal density asymmetries support the choice of a poloidal asymmetry factor $\tilde{\Theta} \sim O(1)$. Using the calculated poloidal density asymmetries to evaluate the gyroviscous torque flow resulted in torque flows in good agreement with experimental values. However, our calculations of the radial dependence of the poloidal density asymmetries do not support the separation-of-variables approximation used in the formulation of the gyroviscous torque flow. When ure eraluate an alternative formulation of the gyroviscous torque flow, which remores this approximation by including the radial gradients of 
the poloidal asymmetries, we find that the added term almost exactly cancels the original term, and we predict values that are at least one order of magnitude smaller than the experimental torque flows, although we note that there is some ambiguity in this formulation. 


\section{Appendix: Neoclassical viscous forces}

For the case $\vec{q}_{j}=0$, we are going to use Braginkii's neoclassical tensor, rewritten for toroidal flux-surface coordinates $[24]$. The toroidal, poloidal and radial projection of the viscous forces have been rewritten in order to show their parametric dependence more explicitly.

- The poloidal component of the parallel viscous force is:

$$
\begin{aligned}
\hat{\theta} \cdot \nabla \cdot \overrightarrow{\vec{\Pi}}_{j}^{0} & \equiv \Pi_{\theta j}^{0}= \\
& \frac{\eta_{0 j} v_{\theta j}}{r^{2}}\left[\epsilon \cos \theta-\frac{2}{3} \frac{\partial \vartheta_{j}}{\partial \theta}+\epsilon\left(\varrho_{0 j}+\frac{\vartheta_{j}}{3}\right) \sin \theta-(\epsilon \sin \theta)^{2}+\right. \\
& \left.\frac{2}{3} \vartheta_{j}\left(\vartheta_{j}-\varrho_{0 j}\right)\right]+\eta_{0 j} \frac{\beta A_{j}}{\epsilon r}\left(4 \epsilon \sin \theta-\varrho_{0 j}-\frac{1}{A_{j}} \frac{\partial A_{j}}{\partial \theta}\right)
\end{aligned}
$$

with

$$
\begin{aligned}
A_{j} & \equiv \frac{1}{B_{\theta} R} \frac{\partial}{\partial \theta}\left(\frac{1}{e_{j} n_{j}} \frac{\partial p_{j}}{\partial r}+\frac{\partial \Phi}{\partial r}\right)-\Omega_{\phi j o} \frac{\partial U_{j}}{\partial \theta} \\
& =\frac{1}{B_{\theta} R}\left[\frac{T_{j}}{\epsilon_{j}} \frac{\partial \vartheta_{j}}{\partial r}+\frac{1}{e} \frac{\partial}{\partial r}\left(T_{e} \vartheta_{e}\right)\right]+\Omega_{\phi j 0} U_{j c} \sin \theta-\Omega_{\phi j 0} U_{j,} \cos \theta \\
\varrho_{0 j} & \equiv \frac{1}{\eta_{0 j}} \frac{\partial \eta_{0 j}}{\partial \theta} \quad \vartheta_{j} \equiv \frac{1}{n_{j}} \frac{\partial n_{j}}{\partial \theta}
\end{aligned}
$$

For a i-I plasma, with both ions species in banana regime, $\varrho_{0 j} \sim\left(\tilde{n}_{j}+\tilde{n}_{I}\right)$.

The FSA of Eq.(A.1)/( $\left.h n_{j}\right)$ is:

$$
\begin{aligned}
<\frac{\Pi_{\theta j}^{0}}{h n_{j}}> & =\frac{\eta_{0 j} v_{\theta j}}{n_{j} R^{2}}\left[-\frac{3}{2}+\frac{\tilde{n}_{j s}}{2 \epsilon}+\frac{\tilde{n}_{I s}}{2 \epsilon}+\frac{\tilde{n}_{I c}}{2 \epsilon}-\frac{\tilde{n}_{j c}}{\epsilon}\right. \\
& \left.+\frac{1}{3 \epsilon^{2}}\left(\tilde{n}_{I c} \tilde{n}_{j c}+\tilde{n}_{I s} \tilde{n}_{j s}+\tilde{n}_{j c} \tilde{n}_{I s}-\tilde{n}_{j s} \tilde{n}_{I c}\right)\right] \\
& +\frac{\eta_{0 j} \beta}{2 n_{j} r}\left[A_{j s}\left(3-\frac{\tilde{n}_{I s}+\tilde{n}_{j s}+\tilde{n}_{j c}}{\epsilon}\right)-A_{j c} \frac{\tilde{n}_{I c}+\tilde{n}_{j c}-\tilde{n}_{j s}}{\epsilon}\right]
\end{aligned}
$$


- The poloidal component of the gyroviscous force is:

$$
\begin{aligned}
\hat{\theta} \cdot \nabla \cdot \overrightarrow{\tilde{\Pi}}_{j}^{3,4} & \equiv \Pi_{\theta j}^{3,4}= \\
& \frac{\eta_{4 j}}{2}\left[-\beta\left(13 \sin \theta-3 \frac{\vartheta_{j}}{\epsilon}\right) \frac{\partial \Omega_{\phi j}}{\partial r}+\mathcal{P}_{1 j} \frac{\beta}{\epsilon} \frac{A_{j}}{r}-2 \frac{\beta}{\epsilon} \frac{\partial A_{j}}{\partial r}+\right. \\
& \left.\frac{v_{\theta j}}{r R}\left(-\mathcal{P}_{2 j} \sin \theta+\mathcal{P}_{3 j} \frac{\vartheta_{j}}{\epsilon}\right)\right]
\end{aligned}
$$

with

$$
\begin{aligned}
& \mathcal{P}_{1 j} \equiv 2+\frac{r}{L_{q}}-\frac{r}{L_{p_{j}}}+3 \epsilon \cos \theta \quad\left(L_{x}^{-1} \equiv-\frac{1}{x} \frac{\partial x}{\partial r}\right) \\
& \mathcal{P}_{2 j} \equiv 4+6 \frac{r}{L_{q}}-3 \frac{r}{L_{p_{j}}}+2 \frac{r}{L_{v_{\theta j}}}+16 \epsilon \cos \theta \quad q=\epsilon_{0} / \beta \\
& \mathcal{P}_{3 j} \equiv 5+3 \frac{r}{L_{q}}-2 \frac{r}{L_{p_{j}}}+2 \frac{r}{L_{\vartheta_{j}}}+\frac{r}{L_{v_{\theta j}}}+7 \epsilon \cos \theta
\end{aligned}
$$

The FSA of Eq.(A.5)/(hn $)$ is:

$$
<\frac{\Pi_{\theta j}^{3,4}}{h n_{j}}>\sim \frac{\beta}{4} \frac{T_{j}}{\omega_{c j}}\left(-13 \frac{\partial \Omega_{\phi j s}}{\partial r}+3 \frac{\tilde{n}_{j c}}{\epsilon} \frac{\partial \Omega_{\phi j s}}{\partial r}-3 \frac{\tilde{n}_{j s}}{\epsilon} \frac{\partial \Omega_{\phi j c}}{\partial r}\right)
$$

- The toroidal component of the parallel viscous force is:

$$
\begin{aligned}
\hat{\phi} \cdot \nabla \cdot \overrightarrow{\vec{\Pi}}_{j}^{0} & \equiv \Pi_{\phi j}^{0}= \\
& \frac{\beta \eta_{0}}{r^{2}}\left\{v_{\theta j}\left[-3 \epsilon \cos \theta+2 \frac{\partial \vartheta_{j}}{\partial \theta}+\epsilon\left(\vartheta_{j}-3 \varrho_{0 j}\right) \sin \theta+2 \vartheta_{j}\left(\varrho_{0 j}-\vartheta_{j}\right)\right]+\right. \\
& \left.3 A_{j} \beta R\left(\varrho_{0 j}-3 \epsilon \sin \theta+\frac{1}{A_{j}} \frac{\partial A_{j}}{\partial \theta}\right)\right\}
\end{aligned}
$$

- The toroidal component of the gyroviscous force is:

$$
\begin{aligned}
\hat{\phi} \nabla \cdot \overrightarrow{\bar{\Pi}}^{3.4} & \equiv \Pi_{\phi j}^{3.4}= \\
& \frac{\eta_{4 j} j}{r R}\left[\mathcal{E}_{j} \frac{v_{\theta}}{\beta}\left(2 \sin \theta-\frac{\vartheta_{j}}{\epsilon}\right)+v_{\phi j} \mathcal{F}_{j}\left(4 \sin \theta-\frac{\vartheta_{j}}{\epsilon}\right)-\frac{1}{\epsilon} A_{j} R \mathcal{E}_{j}\right]
\end{aligned}
$$


with

$$
\mathcal{E}_{j} \equiv-\frac{r}{\eta_{4 j} R^{3}} \frac{\partial \eta_{4 j} R^{3}}{\partial r}, \quad \mathcal{F}_{j} \equiv-\frac{r R}{v_{\phi j}} \frac{\partial \Omega_{\phi j}}{\partial r}
$$

- The radial component of the parallel viscous force is:

$$
\begin{aligned}
\hat{r} \cdot \nabla \cdot \overrightarrow{\vec{\Pi}}_{j}^{0} \quad & \equiv \Pi_{r j}^{0}= \\
& \frac{\eta_{0 j}}{r}\left\{\frac { v _ { \theta j } } { r } \left[-\left(\epsilon \sin \theta-\frac{2}{3} \vartheta_{j}\right) \frac{r}{L_{\eta_{0 j}}}-r^{2} \frac{2}{3} \frac{\partial}{\partial r}\left(\frac{\vartheta_{j}}{r}\right)\right.\right. \\
& \left.+\epsilon^{2} \sin 2 \theta-2 \epsilon \vartheta_{j} \cos \theta\right]+\frac{\partial v_{\theta j}}{\partial r}\left(\epsilon \sin \theta-\frac{2}{3} \vartheta_{j}\right) \\
- & \left.\frac{\beta A_{j}}{\epsilon}\left(4 \epsilon \cos \theta-\frac{r}{L_{\eta_{0 j}}}-\frac{r}{L_{A_{j} / r}}+\frac{r}{L_{q}}\right)\right\}
\end{aligned}
$$

- The radial component of the gyroviscosity is:

$$
\begin{aligned}
\hat{r} \cdot \nabla \cdot \overrightarrow{\vec{\Pi}}_{j}^{3,4} \equiv & \Pi_{r j}^{3,4}= \\
& \frac{\eta_{4 j}}{2}\left[\frac{v_{\theta j}}{r^{2}}\left(-2 \epsilon \cos \theta+2 \frac{\partial \vartheta_{j}}{\partial \theta}-2 \epsilon \vartheta_{j} \sin \theta+1-\frac{r}{L_{\eta_{4 j}}}\right)-\right. \\
& \frac{1 \partial v_{\theta j}}{r \partial r}\left(\epsilon \cos \theta+1-\frac{r}{L_{\eta_{4 j}}}\right)-\frac{\partial^{2} v_{\theta j}}{\partial r^{2}}+ \\
& \frac{A_{j}}{r} \frac{\beta}{\epsilon}\left(\vartheta_{j}-3 \epsilon \sin \theta+\frac{\partial A_{j}}{A_{j} \partial \theta}\right)+ \\
& \left.\frac{\partial \Omega_{\phi j}}{\partial r} \frac{\beta}{\epsilon}\left(6 \epsilon \cos \theta-2-\frac{r}{L_{\eta_{4 j}}}\right)+\beta R \frac{\partial^{2} \Omega_{\phi j}}{\partial r^{2}}\right]
\end{aligned}
$$

- The terms from the perpendicular viscosity tensor can be neglected because $\eta_{2 j} \ll \eta_{4 j}, \eta_{0 j}$ 


\section{References}

[1] S. Suckewer et al., Phys. Rev. Lett. 43, 207 (1979).

[2] S. Suckewer et al., Nucl. Fusion 21, 1301 (1981).

[3] K. Brau et al., Nucl. Fusion 23, 1643 (1983).

[4] R.C. Isler et al., Nucl. Fusion 26, 391 (1986) ; R.C. Isler and L.E. Murray, Appl. Phys. Lett. 42, 355 (1983).

[5] K.H. Burrell et al., Nucl. Fusion 28, 3 (1988).

[6] R.J. Groebner et al., Nucl. Fusion 26, 543 (1986).

[7] H. St. John et al., "Analysis of toroidal rotation data for the DIII-D tokamak", General Atomics Report GA-A19563 (January 1989).

[8] A. Kallenback et al., "Momentum confinement studies on ASDEX", Proc. 16th Europ. Conf. Cont. Fusion and Plasma Phys.,Venice, 1989.

[9] D.E. Roberts et al., "Momentum confinement of ASDEX plasmas during co and counter neutral beam injection", Proc. 15th Europ. Conf. Cont. Fusion and Plasma Phys., Dubrovick, 1988.

[10] A. Kallenback et al., Nucl. Fusion 30, 645 (1990).

[11] M. Bitter et al., Plasma Phys. and Cont. Fusion 29, 1234 (1987).

[12] M. Murakami et al., Plasma Phys. and Cont. Fusion 28, 17 (1986).

[13] S.D. Scott et al., "Measurements of toroidal rotation on TFTR", Proc. 14th Europ. Conf. Cont. Fusion and Plasma Phys., Madrid, 1987.

[14] S.D. Scott et al., "Analysis of rotation speed radial profiles on TFTR", Proc. 15th Europ. Conf. Cont. Fusion and Plasma Phys., Dubrovick, 1988. 
[15] S.D. Scott et al., "Current drive and confinement of angular momentum in TFTR", Proc. 12th Int. Conf. Plasma Phys, and Cont. Nucl. Fusion, Nice, 1988 (IAEA-CN-50/E-III-5).

[16] S.D. Scott et al., Phys. Fluids B 2, 1304 (1990).

[17] S.D. Scott et al., Phys. Rev. Lett. 64, 531 (1990).

[18] D. Stork et al., "Momentum transport and scaling effects observed in neutral beam heated rotating plasmas in JET", Proc. 14th Europ. Conf. Cont. Fusion and Plasma Phys., Madrid, 1987.

[19] N.C. Hawkes et al., "Profiles of toroidal plasmas rotation", Proc. 15th Europ. Conf. Cont. Fusion and Plasma Phys., Dubrovick, 1988.

[20] H.P.L. de Esch et al., "Toroidal Plasma Rotation in JET", Joint European Torus Report JET-P(90)14 (June 1990).

[21] W.M. Stacey, Nucl. Fusion 31, 31 (1991).

[22] J.A. Snipes et al., "Plasma stored energy and momentum losses during large MHD activity in JET", Joint European Torus Report JET-P(89)64 (September 1989).

[23] N. Asakura et al., Bull. Am. Phys. Soc. 34, 2049 (1989).

[24] W.M. Stacey and D.J. Sigmar, Phys. Fluids 28, 2800 (1985).

[25] W.M. Stacey et al., Nucl. Fusion 26, 293 (1986).

[26] A.A. Ware, Phys. Rev. Lett. 64, 2655 (1990).

[27] C.T. Hsu and D. Sigmar, Plasma Phys. and Cont. Fusion 32, 499 (1990). 
[28] W.M. Stacey and G.W. Neeley, "Neoclassical theory of momentum transport by marginally collisional ions in strongly-rotating tokamak plasmas with unbalanced neutral beam injection", Georgia Tech Report GTFR 96 (1990).

[29] D.Kh. Morozov and O.P. Pogutse, "Relaxation of plasma toroidal rotation in tokamaks and anomalous viscosity", Proc. 11th Int. Conf. Plasma Phys. and Cont. Nucl. Fusion, Kyoto, 1986.

[30] B.B. Kadomtsev and O.P. Pogutse, "Electron heat conductivity of the plasma across a 'braided' magnetic field", Proc. 7th Int. Conf. Plasma Phys, and Cont. Nucl. Fusion, Innsbruck, 1978.

[31] D.K. Morozov and O.P. Pogutse, Sov. J. Plasma Phys. 12(6), 381 (1986).

[32] D.Kh. Morozov et al., Sov. J. Plasma Phys. 14, 147 (1988).

[33] M.V. Osipenko et al., "Damping of toroidal rotation by anomalous ion viscosity", Proc. 12th Int. Conf. Plasma Phys, and Cont. Nucl. Fusion, Nice, 1988 (IAEACN-50/D-4-4).

[34] D.L. Brower, Phys. Rev. Lett. 59, 48 (1987).

[35] N. Mattor and P.H. Diamond, Phys. Fluids 31, 1180 (1988).

[36] G.S. Lee and P.H. Diamond, Phys. Fluids 29, 3291 (1986).

[37] H. Biglari et al., "Advances in the theory of ion-temperature-gradient-driven turbulence", Proc. 12th Int. Conf. Plasma Phys. and Cont. Nucl. Fusion, Nice, 1988 (IAEA, Vienna, 1989; Vol 2, p. 261).

[38] R. Dominguez and R.E. Waltz, Nucl. Fusion 29, 885 (1989).

[39] F. Romanelli, Phys. Fluids B5, 1018 (1989).

[40] T. Hahm and W. Tang, Phys. Fluids B1, 1185 (1989). 
[41] N. Mattor and P.H. Diamond, Phys. Fluids B1, 1980 (1989).

[42] R.B. Howell, Rev. Sci. Instrum. 59, 1521 (1988).

[43] H. Park, Princeton Plasma Physics Laboratory Report PPPL-2592 (1989).

[44] G. Taylor et al., Rev. Sci. Instrum. 55, 1739 (1984).

[45] M. Bitter et al., Rev. Sci. Instrum. 59, 1852 (1988).

[46] A.T. Ramsey and S.L. Turner, "HAIFA: Modular, Fiber-Optic Coupled, Spectroscopic Diagnostic for Plasmas", Princeton Plasma Physics Laboratory Report PPPL-2407.

[47] K.W. Hill, private communication (PPPL, 1988).

[48] R.V. Budney et al., "Deuterium recycling, confinement, and limiter flux in TFTR", Princeton Plasma Physics Laboratory Report PPPL-2705 (July 1990).

[49] H.W. Hendel et al., Rev. Sci. Instrum, 59, 1682 (1988).

[50] K.W. Hill et al., Rev. Sci. Instrum. 56, 83 (1985).

[51] W.M. Stacey, Fusion Plasma Analysis (Wiley-Interscience Publ., 1981), p. 78.

[52] R.J. Goldston, "Topics in confinement analysis of tokamaks with auxiliary heating", Proc. of Course and Workshop on "Basic physical processes of toroidal fusion plasmas", Varenna, 1985 (Monotypia Franchi, Italy, 1985), p .165.

[53] R.H. Radeztsky et al., Proc. 15th Europ. Conf. Contr. Fusion and Plasma Phys., Dubrovick, 1988 (EPS 1988) Vol.1, p.79.

[54] W.M. Stacey, Plasma Phys. Cont. Fusion 31, 1451 (1989).

[55] G. Pautasso and W.M. Stacey, "A review of momentum confinement in tokamak plasmas", Georgia Tech Report GTFR 95 (January 1991). 
[56] G. Pautasso, Analysis of rotation experiments in TFTR, P.h.D. Thesis, Georgia Institute of Technology, December 1991.

[57] W.M. Stacey, Nucl. Fusion 30, 30, 2435(1990).

[58] D.W. Ross et al., "IPSG Panel Report: Thermal and particle transport in tokamaks. Theoretical models for ignition studies. Version 2.2" (6/1987); also Horton's private communication to D.J.Sigmar and C.T.Hsu, Proc. 12th Int. Conf. Plasma Phys. and Cont. Nucl. Fusion, Nice, 1988 (IAEA-CN-50/D-2-3-3).

[59] TRANSP ref.

[60] A.J. Wootton, "Edge Turbulence", The University of Texas Report FRCR 371 (1990).

[61] Y. Nagayama, private communication (PPPL, March 1991).

[62] J.D. Callen, "Transport Models; Anomalous Transport", School on Plasma Turbulence and Transport, Madison, Wisconsin (19-23 August 1991).

[63] W.M. Stacey, Nucl. Fusion, 27, 1213 (1987).

[64] J. Mathew et al., "Investigation of the Electric Field Structure in ISX-B Plasmas", Oak Ridge Nationa Laboratory Report ORNL/TM-9386 (April 1986).

[65] A.J. Wootton et al., Phys. Fluids B2 12, 2879 (1990).

[66] D.W. Ross et al., "On Standard Forms for Transport Equations and Fluxes Part II", The University of Texas Report FRCR 357 (March 1990).

[67] R.E. Waltz, Phys. Fluids 25, 1269 (1982).

[68] H. Hsuan, et al., Bull. Am. Phys. Soc. 35, 2084(1990).

[69] R.J. Groebner, et al., Phys. Rev. Lett. 64, 3015(1990). 
[70] K. Ida and S. Hidekuma, Phys. Rev, Lett, 65, 1364(1990).

[71] K.H. Burrell et al., Phys. Rev, Lett, 47, 511 (1981).

[72] C.S. Chang and R.D. Hazeltine, Nucl. Fusion 20, 1397 (1980).

[73] W.M. Stacey and D.J. Slgmar, Phys. Fluids 27, 2076 (1984).

[74] W.M. Stacey et al., Nucl. Fusion 25, 463 (1985).

[75] C.S. Chang and R.W. Harvey, Nucl. Fusion 23, 935 (1983). 


\section{List of Figures}

1 Measurements of $v_{\phi}(R)$ by CHERS and XCS, $\ldots \ldots \ldots \ldots 7$

2 Time histortes of several plasma parameters $\left(T_{i}(0)\right.$ and $v_{\phi}(0)$ as measured by the XCS, $P_{b}, \int n_{a}$ dl, neutron emission and total stored energy, $W_{\text {tot }}$, diamagnetic) for co-injection and counter-injectlon, both with $P_{b}=11.6 \mathrm{MW}, I_{p}=1,1 \mathrm{MA}, \mathrm{B}=4.75$ T'esla. $\ldots \ldots \ldots$.

3 Central rotation velocity, ?wix $(0)$, as maasured by CHERS, versus input torque per particle $\left(\square I_{p}=1-1.1 . M A ; \square: I_{p}=1.7-1.8 M A ; x\right.$ : $B<4$ Tesla $\ldots \ldots \ldots \ldots \ldots \ldots \ldots, \ldots \ldots, \ldots \ldots$

4 Radial profles of $v_{\phi j} / v_{t h j}$ and $v_{j}^{*}, j=1, I(1=$ deuterim, $I=$ carbon $), 70$

5 Measured plasma parameters profiles at $P_{D}=11.6 \mathrm{MW}$ for co-injection (一) and counter-injection $(\ldots), \ldots \ldots \ldots \ldots \ldots$

6 Experimental thermal momentum conflnement time, $\tau_{\phi t h}^{n: x}$, versus input power. ( $\triangle$ : co-injection shots at $I_{p}=1-1.1 \mathrm{MA} ; \Delta_{\text {: co-Injection shots }}$ at $I_{p}=1.7-1.8 \mathrm{MA} ; \square$; counter-injection shots at $I_{p}=1-1.1 \mathrm{MA} ; \square$; counter-injection shots at $I_{p}=1.7-1.8 \mathrm{MA}_{i} \times: B<4 T^{\prime}$ esla). . . .

7 Comparison of the toroldal central velocity predicted by the gyroviscous theory $\left(v_{\phi}^{g \prime \prime}(0)\right)$ with the experimental measurements $\left(v_{\phi}^{g x}(0)\right)$. ( a: co-injection shots at $I_{p}=1-1.1 \mathrm{MA} ; 0$ : co-injection shots at $I_{p}=$ 1.7-1.8 MA; $\triangle$ : counter-injection shots at $I_{p}=1-1.1 \mathrm{MA} ; *$ counterinjection shots at $\left.I_{p}=1.7-1.8 \mathrm{MA}\right), \ldots \ldots \ldots \ldots \ldots$

8 Comparison of the experimental, $\tau_{\phi}^{e x}$, and the gyroviscous, $\tau_{\phi}^{g \prime \prime}$, momentum confinement times. ( $\square$ : co-injection shots at $I_{p}=1-1.1 \mathrm{MA}$; o: co-injection shots at $I_{p}=1.7-1.8 \mathrm{MA} ; \Delta$ : counter-injection shots at $I_{p}=1-1.1 \mathrm{MA} ; \star$ : counter-injection shots at $\left.I_{p}=1.7-1.8 \mathrm{MA}\right) . \ldots$ 
9 Scaling of the experimental momentum confinement, $\tau_{\psi}^{\text {en }}$, with the cen tral ion temperature, $T_{i}(0)$. ( $\square$ : co-injection shots at $I_{p^{\prime}}=1.1 .1 \mathrm{MA}_{\text {; }}$ o: co-infection shots at $I_{p}=1.7-1.8 \mathrm{MA} ; \Delta$ : counter-injection shots at $I_{p}=1-1.1 \mathrm{MA} ; *$ counter-injection shots at $\left.I_{p}=1.7-1.8 \mathrm{MA}\right), \ldots$

10 Scaling of the experimental momentum confinement, $\tau_{\phi}^{\text {awd }}$, with the parameter $\bar{Z}$. ( $\square$ : co-Injection shots at $I_{p}=1.1 .1 \mathrm{MA} ; 0$ : co-Injection shots at $I_{p}=1.7-1.8 \mathrm{MA} ; \Delta$ : counter-injection shots at $I_{p}=1-1.1 \mathrm{MA}$; $\star$ : counter-injection shots at $I_{p}=1.7-1.8 \mathrm{MA}$ ).

11 Comparison of experimental torque flow, $K_{\psi}^{\text {e.an }}$, with the theoretical pre. dictions of the gyroviscous theory (gv), of the stochastic magnetic perturbation theory (smp), of the untrapped particle modes theory (up) and of the lon temperature gradient driven modes theory $\left(\eta_{i}\right) \ldots \ldots$

12 Comparison of the toroidal central velocity $\left(v_{\phi}^{g n}(0)\right)$ predicted by the $\hat{Z}=Z_{\text {eff }}$ gyroviscous theory model with the experimental measurements $\left(v_{\phi}^{e x}(0)\right)$, ( $\square$ : co-injection shots at $I_{p}=1-1.1 \mathrm{MA} ; 0$ : coinjection shots at $I_{p}=1.7-1.8 \mathrm{MA} ; \Delta$ : counter-injection shots at $I_{p}=$ 1-1.1 MA; *: counter-injection shots at $\left.I_{p}=1.7-1.8 \mathrm{MA}\right) . \ldots \ldots$

13 Comparison of the experimental $\eta_{i}^{\text {ex }}$ with the theoretical $\eta_{i}^{\text {Rom }}, \eta_{i}^{\text {Hahm }}$ and $\eta_{i}^{M \& D}, \ldots \ldots \ldots \ldots \ldots \ldots \ldots \ldots \ldots \ldots \ldots$

14 Radial profles of $v_{\theta I}(r)$ computed for $\gamma_{I}=0.0$ and 0.1 . The $v_{\theta *}$ is a "trial" function. The $v_{\phi}(r)$ and $\beta v_{\phi}(r)$ profiles are also shown for comparison.

15 Radial profiles of $\tilde{n}_{I_{0}}$ and $\tilde{n}_{I_{s}}$, (a) evaluated for $\gamma_{I}=0.0$ and 0.1 , and (b) assuming $v_{\theta I}=v_{\theta *}$. The parameter $\epsilon$ is also shown for comparison. 81

16 Radial proflies of $v_{\theta i}(r)$ evaluated for $\gamma_{i}=0.0,0.5,0.9$, and $\gamma_{I}=0.1$. The $v_{\phi}(r)$, and $\beta v_{\phi}(r)$ profiles are also shown for comparison. . . . . 82

17 Radial profiles of $\tilde{n}_{i c}$ and $\tilde{n}_{i s}$ evaluated for $\gamma_{i}=0.0,0.5,0.9$, and $\gamma_{I}=0.1$. The parameter $\epsilon$ is also shown for comparison. 
18 Radial proflles of $(\Theta G / Z)_{e f f}$ evaluated for the case $\gamma_{i}=0.9, \gamma_{I}=0.1$, and for the case $\gamma_{i}=0.9(\ldots)$ and $v_{\theta I}=v_{\theta *}(\ldots) \ldots \ldots \ldots 84$

19 Radial proflles of $K_{\phi}^{g \prime \prime}$ (case $\tilde{\Theta}=1,5$ and $\bar{Z}=Z_{e f f}$ ), $\hat{K}_{\phi}^{g \prime \prime}$ and $K_{\phi}^{g \prime *}$ (case $\gamma_{i}=0.9, \gamma_{t}=0.1$ ). The experimental $K_{\phi}^{a x}$ proflle is also shown

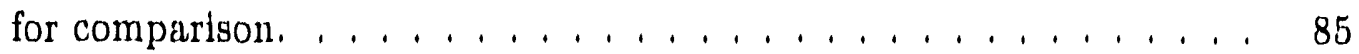

20 Radial profiles of $G_{\eta v_{i}}$ and $G_{\Xi_{1}}$, Eq.(112), for the case $\gamma_{I}=0.1$ and $\gamma_{i}=0.9$. 


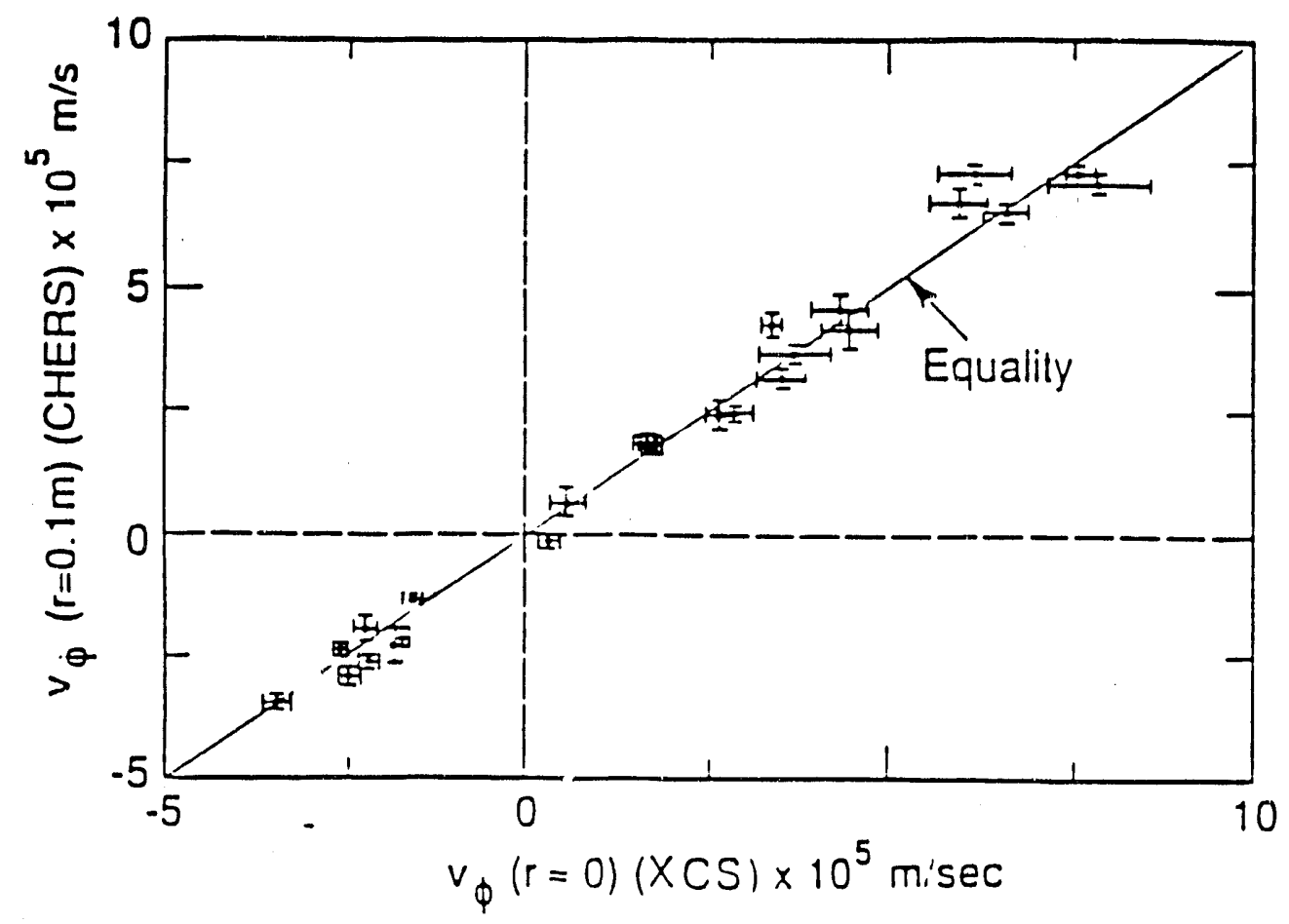

Figure 1: Measurements of $v_{\phi}(R)$ by CHERS and XCS. 

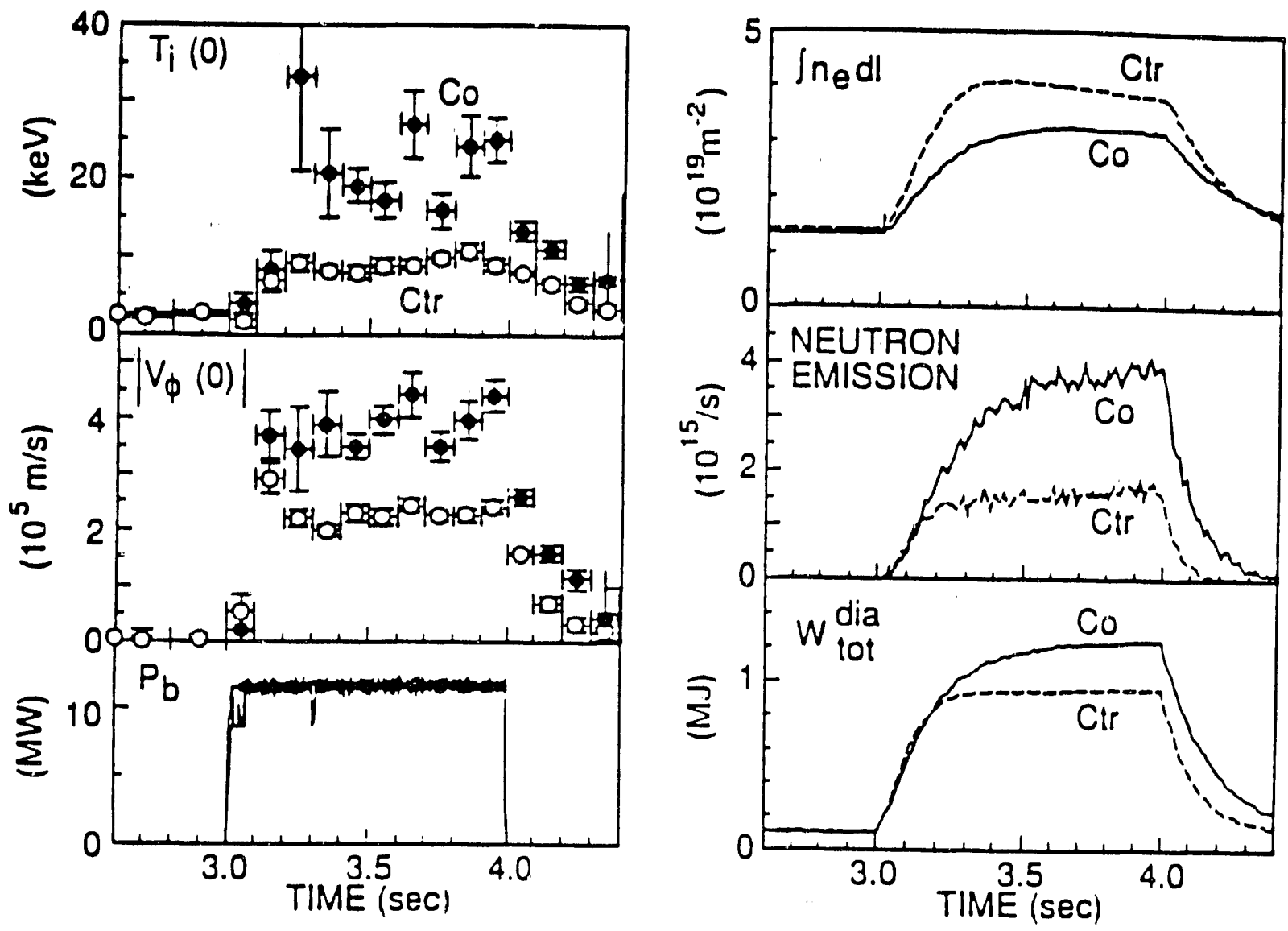

Figure 2: Time histories of several plasma parameters $\left(T_{1}(0)\right.$ and $v_{\phi}(0)$ as measured by the XCS, $P_{b}, \int n_{e} d l$, neutron emission and total stored energy, $W_{t o t}$, diamagnetic) for co-injection and counter-injection, both with $P_{b}=11.6 \mathrm{MW}, I_{p}=1,1 \mathrm{MA}, \mathrm{B}=$ 4.75 Tesla. 


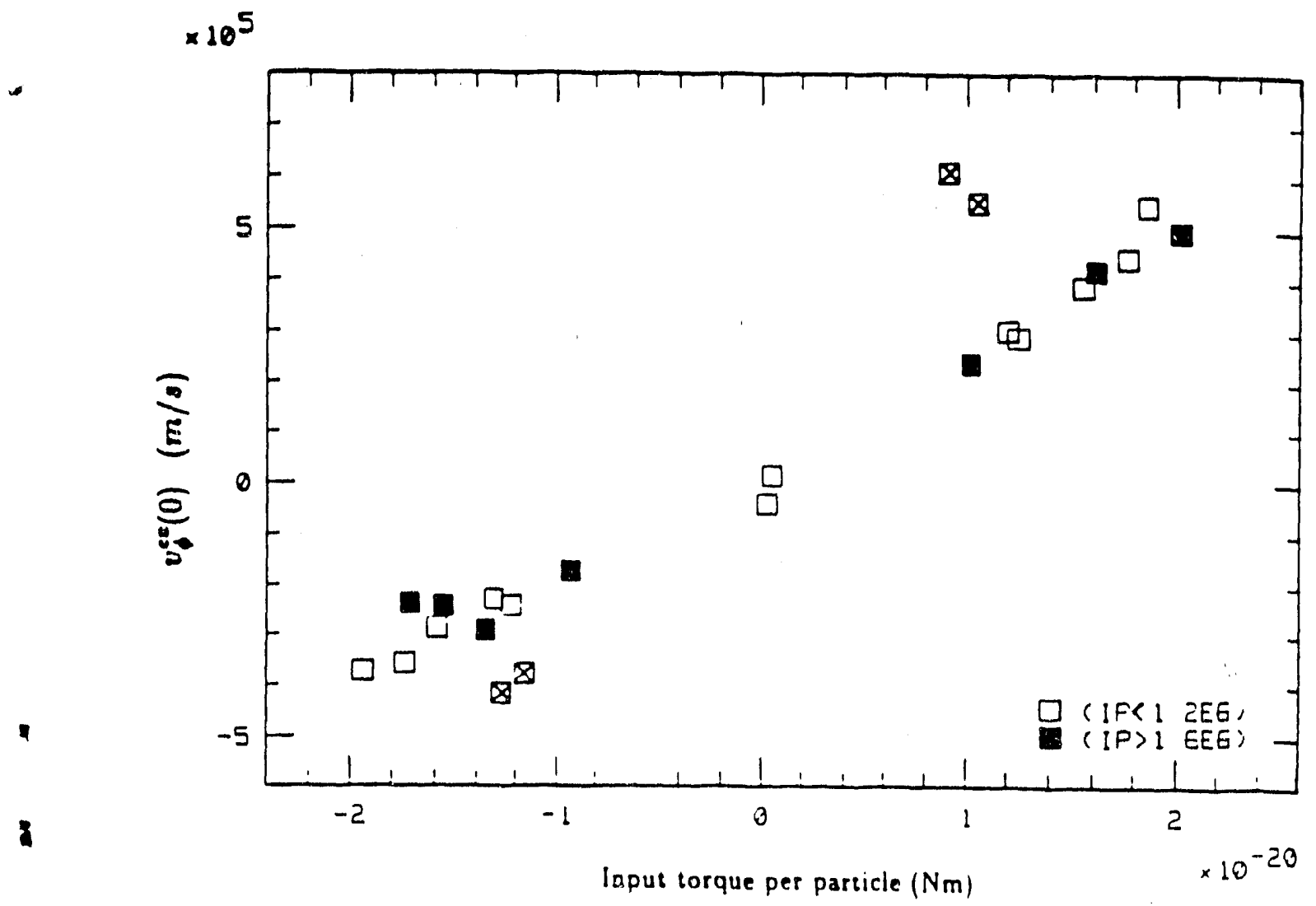

Figure 3: Central rotation velocity, $v_{\phi}^{e x}(0)$, as measured by CHERS, versus input torque per particle $\left(\square I_{p}=1-1.1 M A ; \square: I_{p}=1.7-1.8 M A ; \mathrm{x}: B<4\right.$ Tesla) 


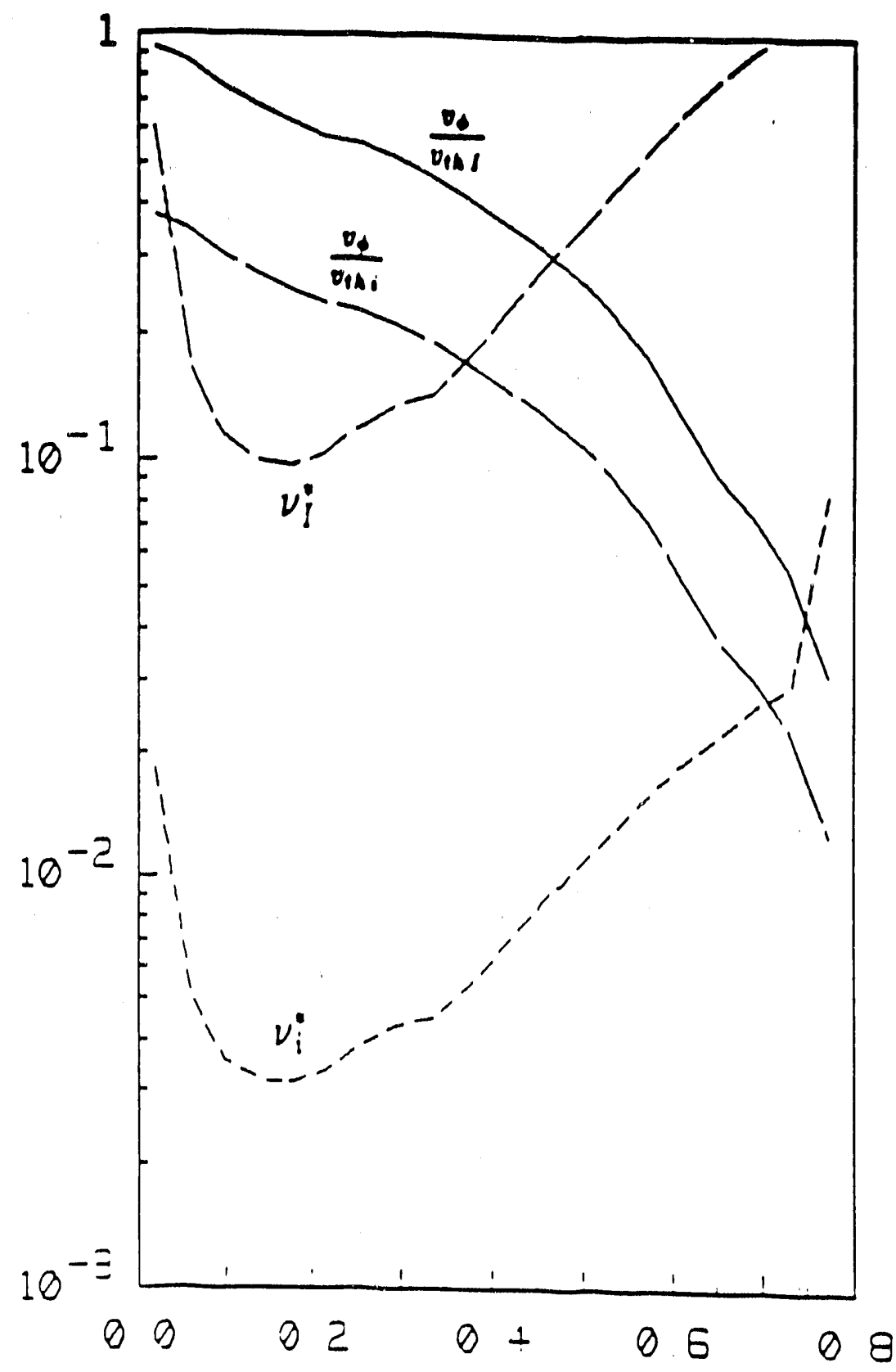

Minor Radius (m)

Figure 4: Radial profiles of $v_{\phi_{j}} / v_{t h j}$ and $\nu_{j}^{*}, \mathrm{j}=\mathrm{i}, \mathrm{I}$ ( $\mathrm{i}=$ deuterium, $\mathrm{I}=$ carbon) 

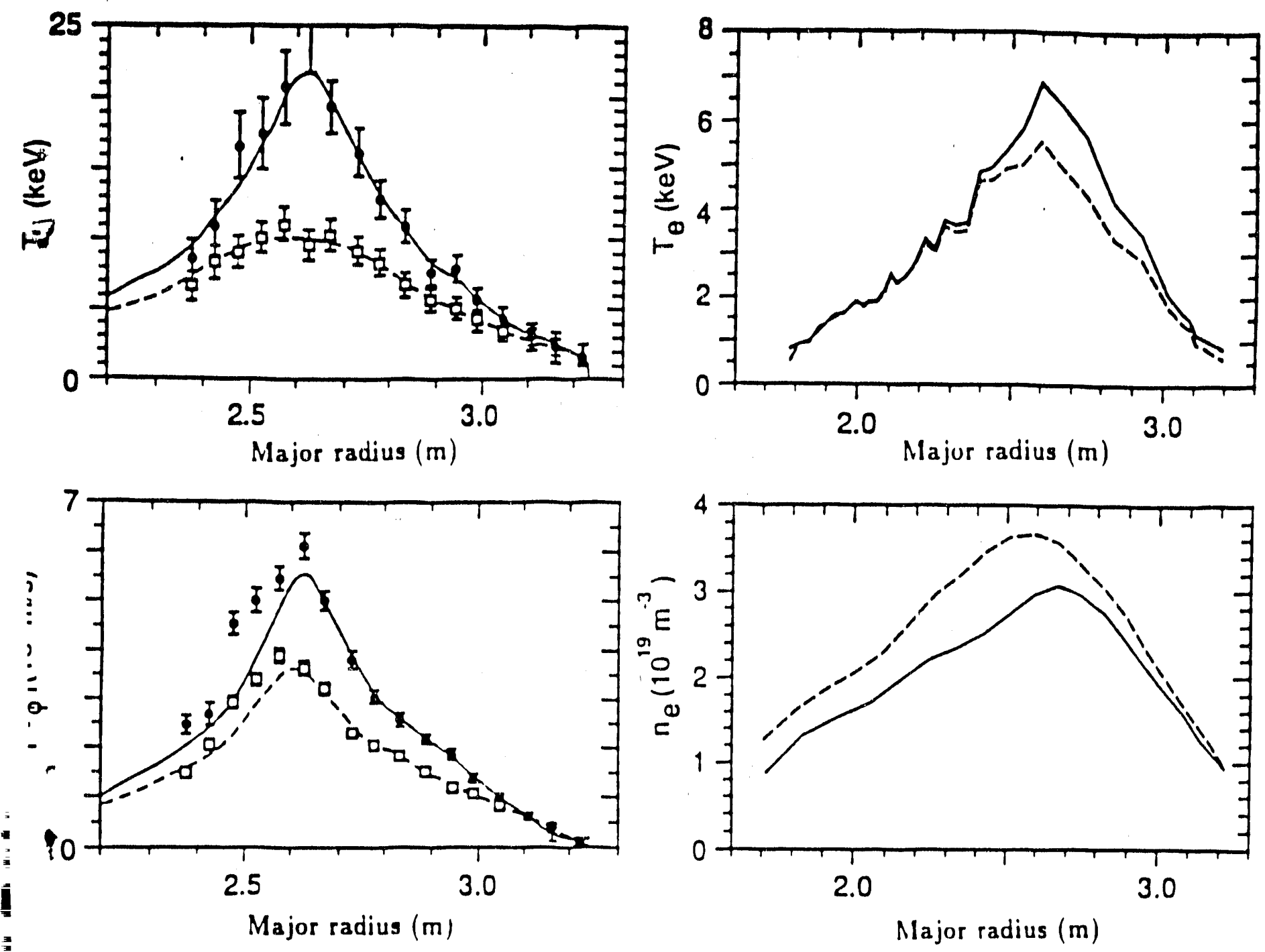

Figure 5: Measured plasma parameters profiles at $P_{B}=11.6 \mathrm{MW}$ for co-injection (—) and counter-injection (- - -). 


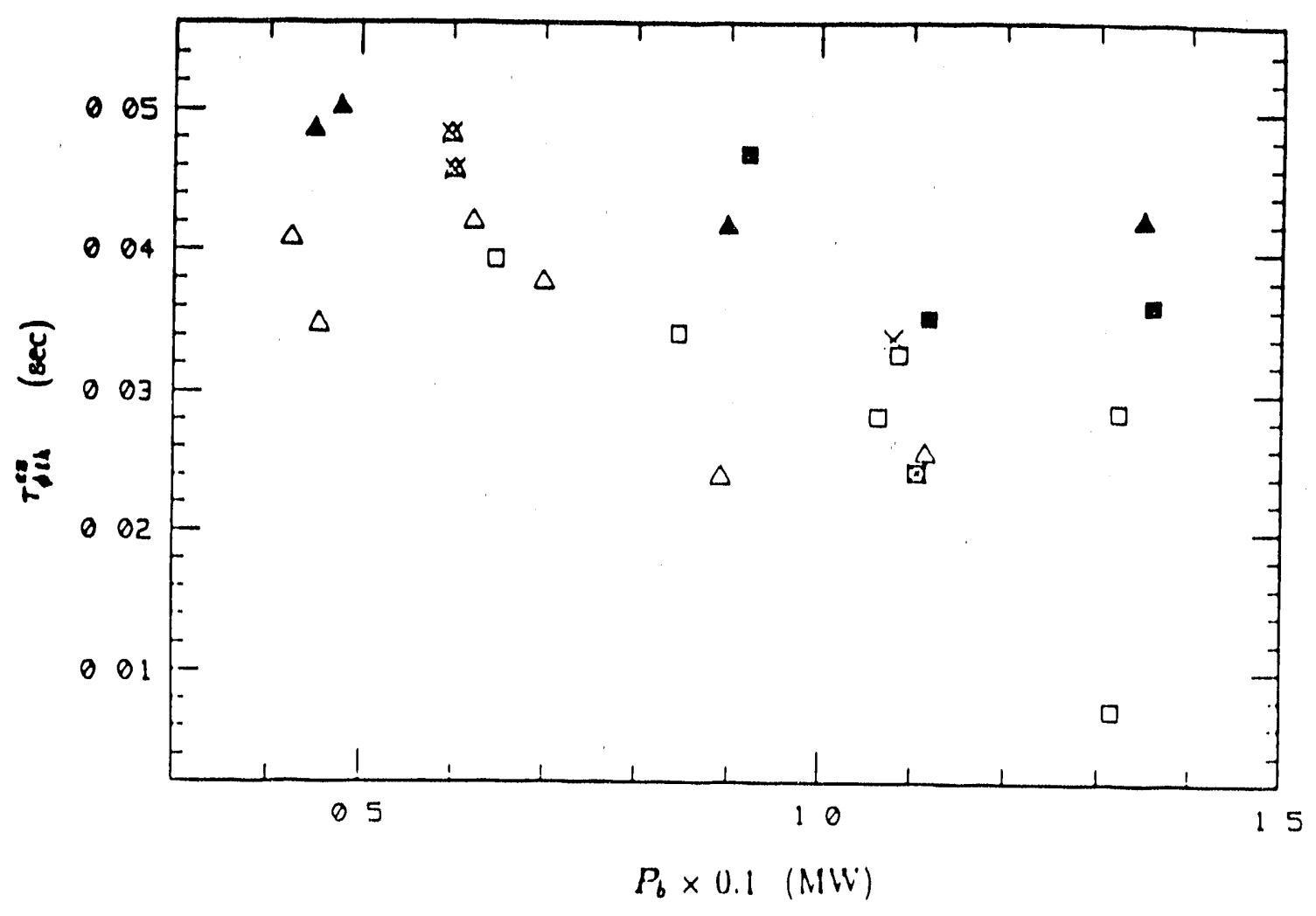

Figure 6: Experimental thermal momentum confinement time, $\tau_{\phi t h}^{e x}$, versus input power. ( $\triangle$ : co-injection shots at $I_{p}=1-1.1 \mathrm{MA} ; \Delta$ : co-injection shots at $I_{p}=$ 1.7-1.8 MA; $\square$ : counter-injection shots at $I_{p}=1-1.1 \mathrm{MA} ; \square$ : counter-injection shots at $I_{p}=1.7-1.8 \mathrm{MA} ; \times: B<4$ Tesla). 


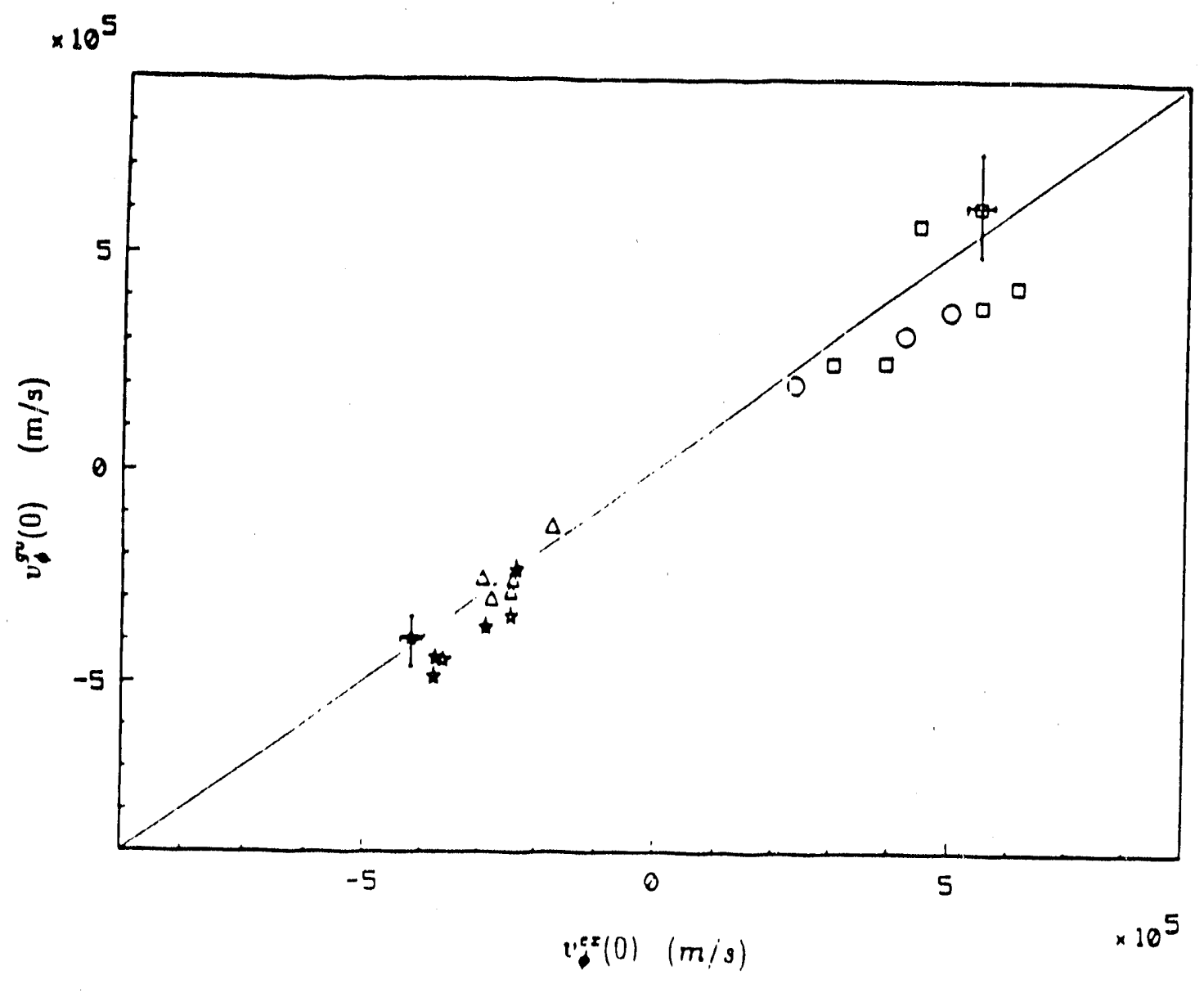

Figure 7: Comparison of the toroidal central velocity predicted by the gyroviscous theory $\left(v_{\phi}^{g \prime}(0)\right)$ with the experimental measurements $\left(v_{\phi}^{e x}(0)\right)$. ( $\square$ : co-injection shots at $I_{p}=1-1.1 \mathrm{MA} ; 0$ : co-injection shots at $I_{p}=1.7-1.8 \mathrm{MA} ; \triangle$ : counter-injection shots at $I_{p}=1-1.1 \mathrm{MA} ; \star$ : counter-injection shots at $I_{p}=1.7-1.8 \mathrm{MA}$ ). 


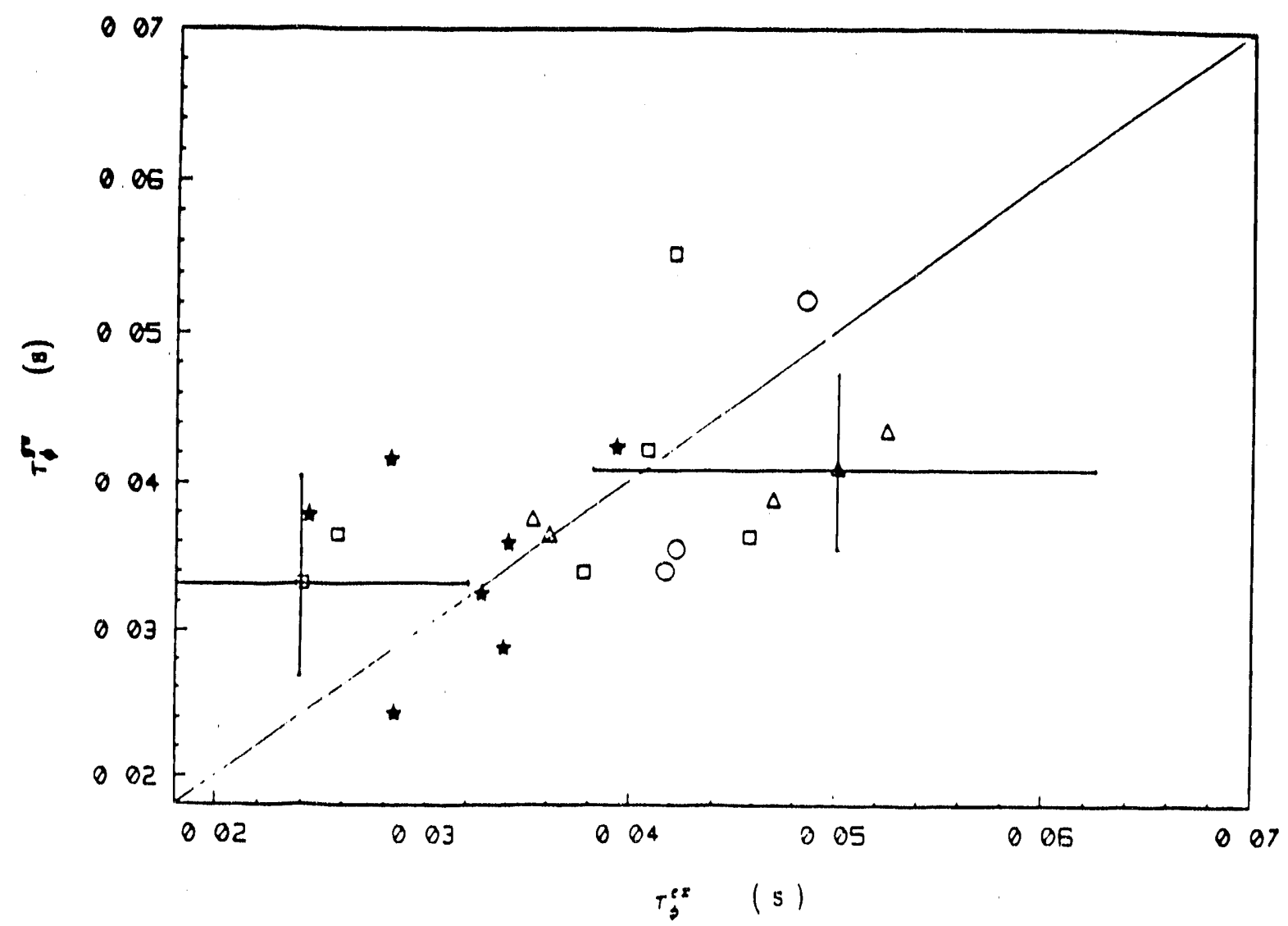

Figure 8: Comparison of the experimental, $\tau_{\phi}^{e x}$, and the gyroviscous, $\tau_{\phi}^{g v}$, momentum confinement times. ( $\square$ : co-injection shots at $I_{p}=1-1.1 \mathrm{MA} ; 0$ : co-injection shots at $I_{p}=1.7-1.8 \mathrm{MA} ; \triangle$ : counter-injection shots at $I_{p}=1-1.1 \mathrm{MA} ; \star$ : counter-injection shots at $I_{p}=1.7-1.8 \mathrm{MA}$ ). 


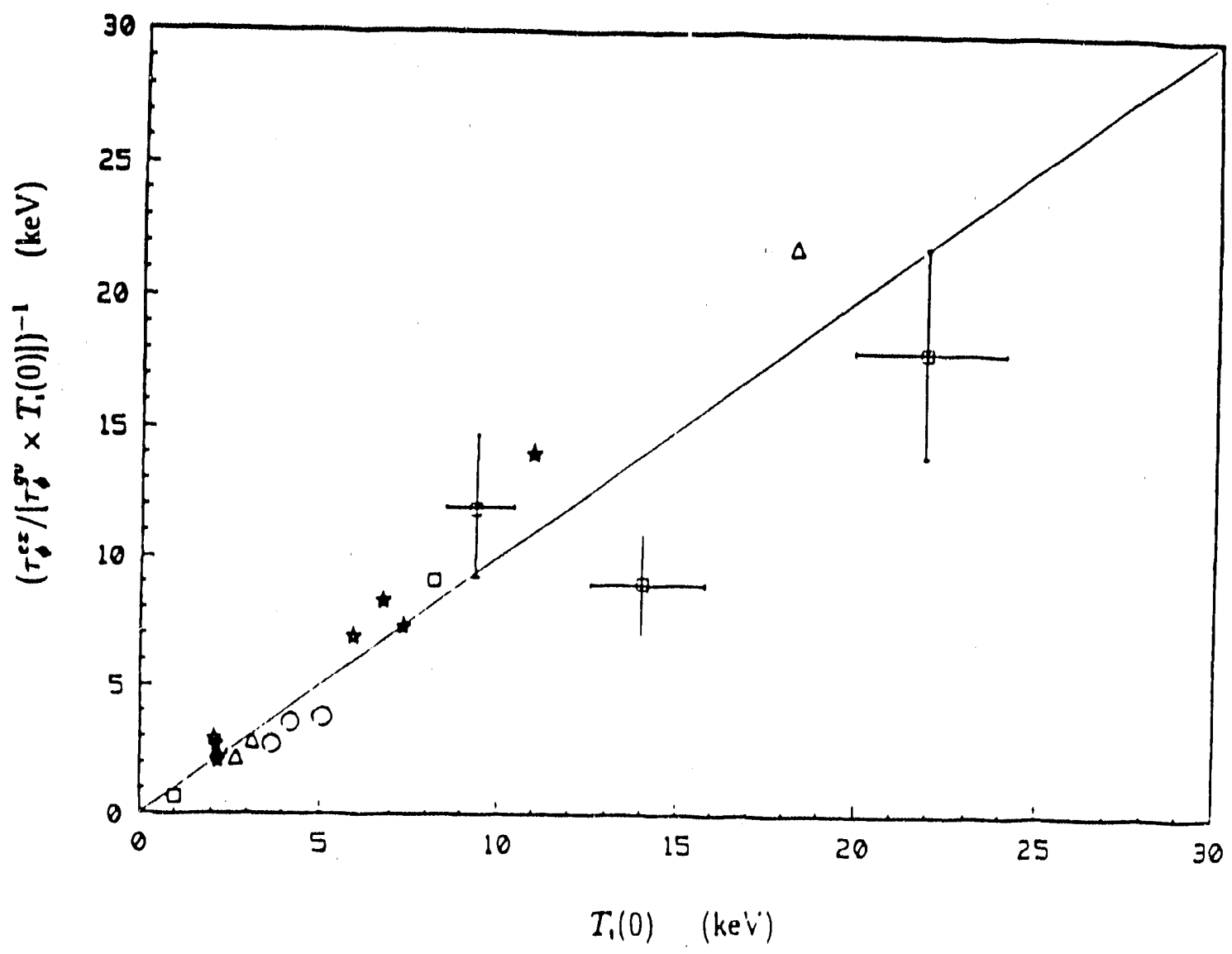

Figure 9: Scaling of the experimental momentum confinement, $\tau_{\phi}^{e x}$, with the central ion temperature, $T_{i}(0)$. ( $\square$ : cö-injection shots at $I_{p}=1-1.1 \mathrm{MA} ; 0$ : co-injection shots at $I_{p}=1.7-1.8 \mathrm{MA} ; \triangle$ : counter-injection shots at $I_{p}=1-1.1 \mathrm{MA} ; \star$ : counter-injection shots at $I_{p}=1.7-1.8 \mathrm{MA}$ ). 


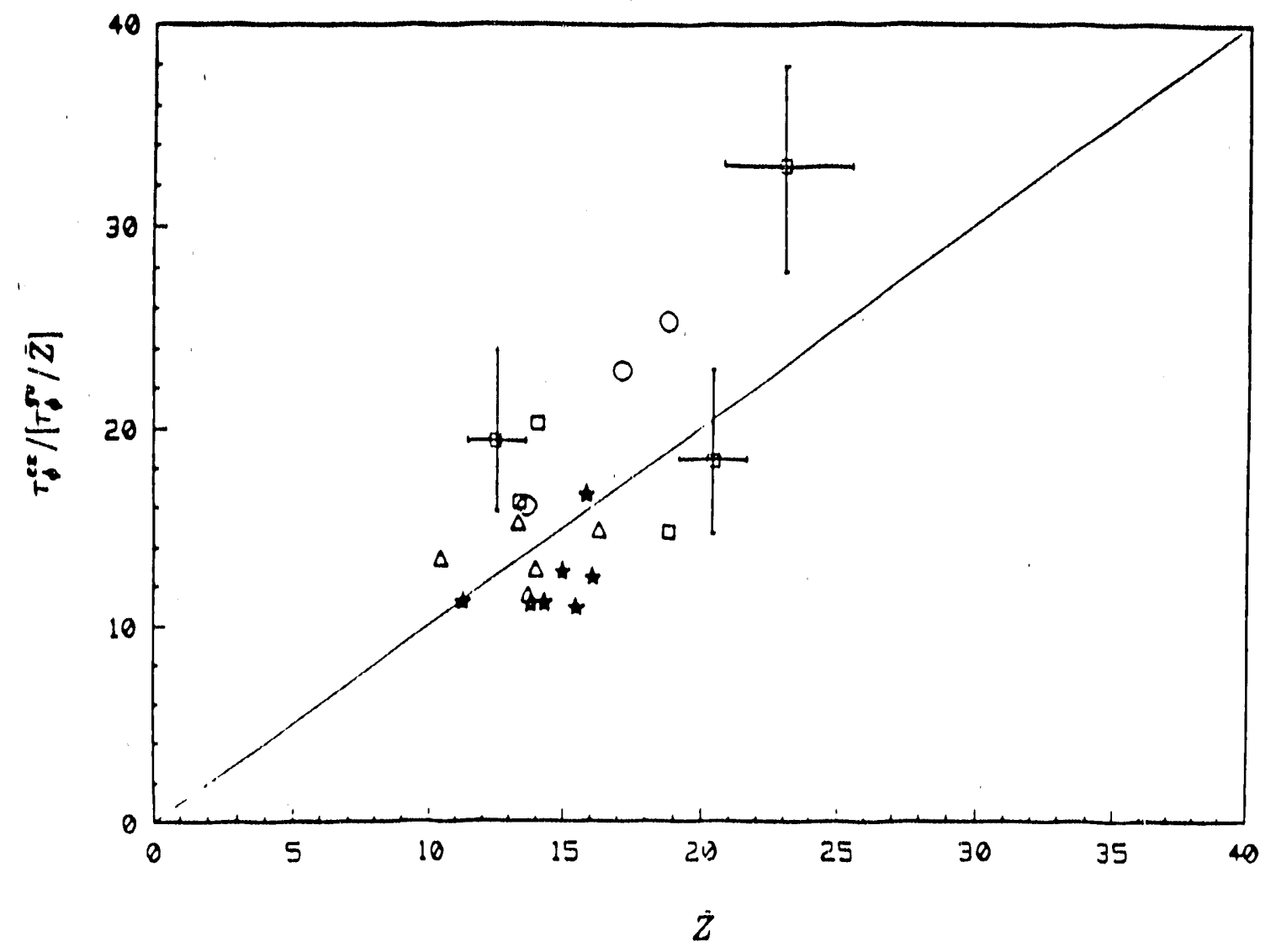

Figure 10: Scaling of the experimental momentum confinement, $\tau_{\phi}^{e x}$, with the parameter $\bar{Z}$. ( $\square$ : co-injection shots at $I_{p}=1-1.1 \mathrm{MA} ; 0$ : co-injection shots at $I_{p}=1.7-1.8$ MA; $\triangle$ : counter-injection shots at $I_{p}=1-1.1 \mathrm{MA} ; \star$ : counter-injection shots at $I_{p}=$ 1.7-1.8 MA). 


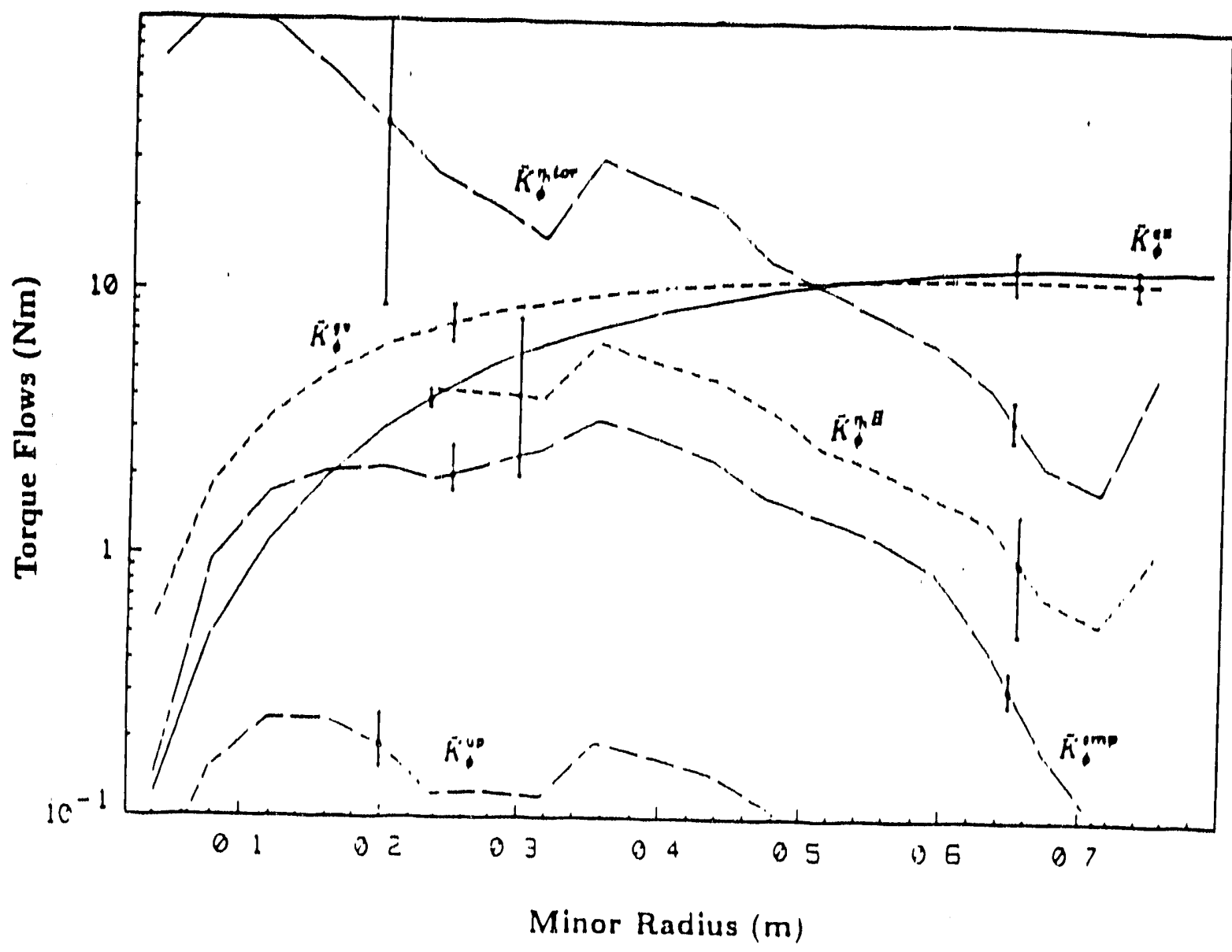

Figure 11: Comparison of experimental torque flow, $K_{\phi}^{e x}$, with the theoretical predictions of the gyroviscous theory (gv), of the stochastic magnetic perturbation theory (smp), of the untrapped particle modes theory (up) and of the ion temperature gradient driven modes theory $\left(\eta_{1}\right)$. 


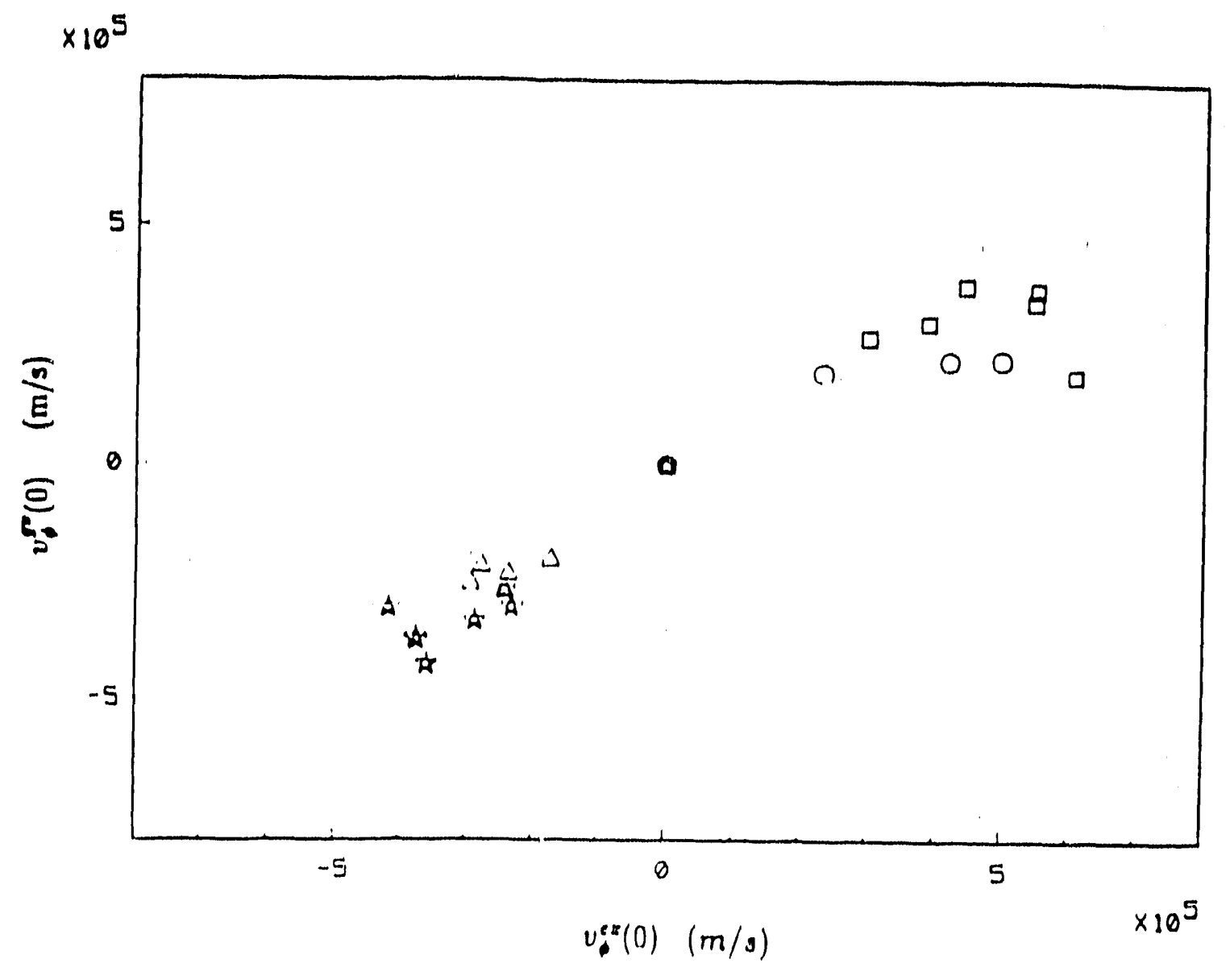

Figure 12: Comparison of the toroidal central velocity $\left(v_{\phi}^{g \prime \prime}(0)\right)$ predicted by the $\hat{Z}=$ $Z_{\text {eff }}$ gyroviscous theory model with the experimental measurements $\left(v_{\phi}^{e x}(0)\right)$. ( $\square$ : co-injection shots at $I_{p}=1-1.1 \mathrm{MA} ; 0$ : co-injection shots at $I_{p}=1.7-1.8 \mathrm{MA} ; \Delta$ : counter-injection shots at $I_{p}=1 \cdot 1.1 \mathrm{MA} ; \star$ counter-injection shots at $I_{p}=1.7-1.8$ MA). 


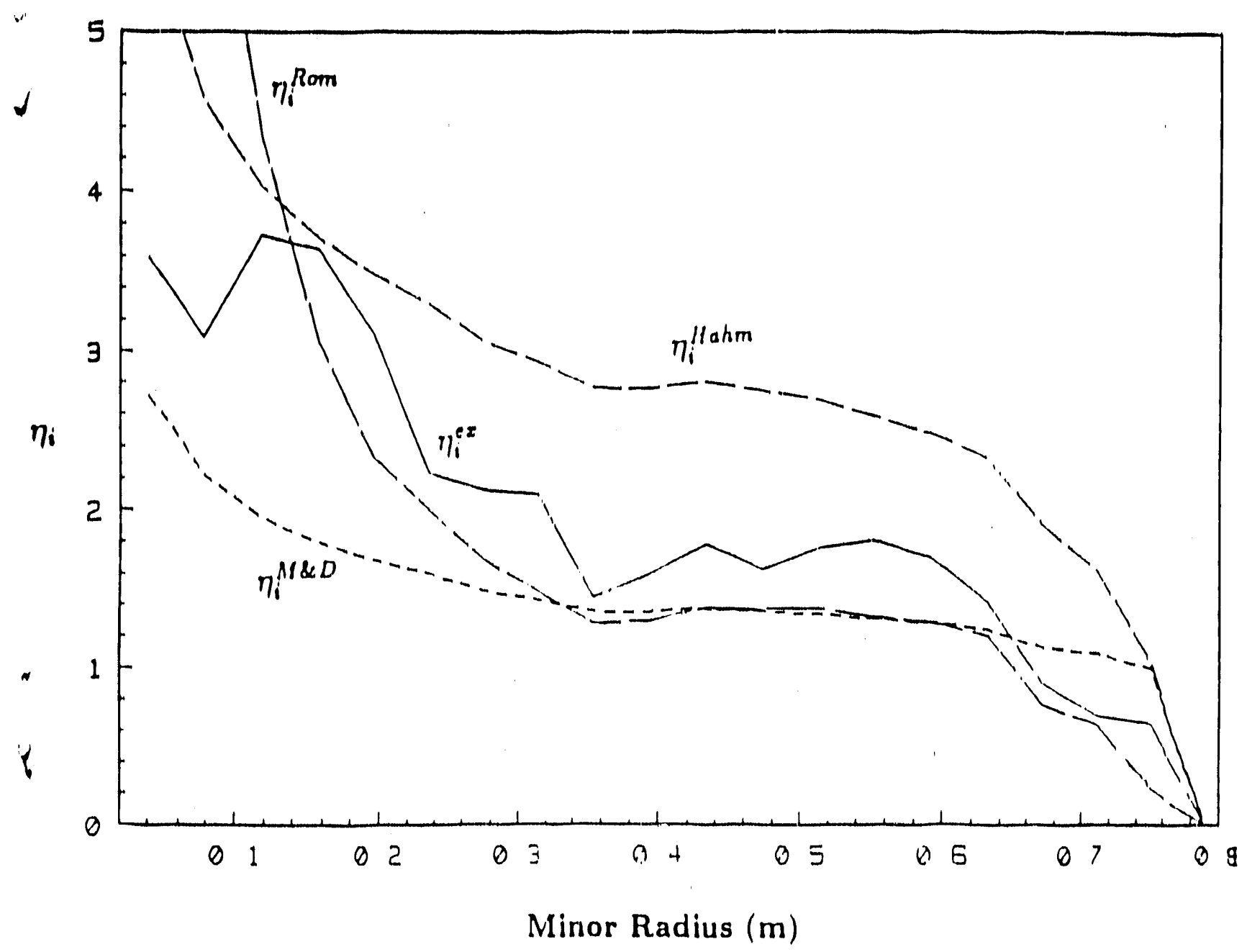

Figure 13: Comparison of the experimental $\eta_{i}^{\text {ex }}$ with the theoretical $\eta_{i}^{\text {Rum }}, \eta_{i}^{\text {Halmm }}$ and $\eta_{i}^{M \& D}$. 


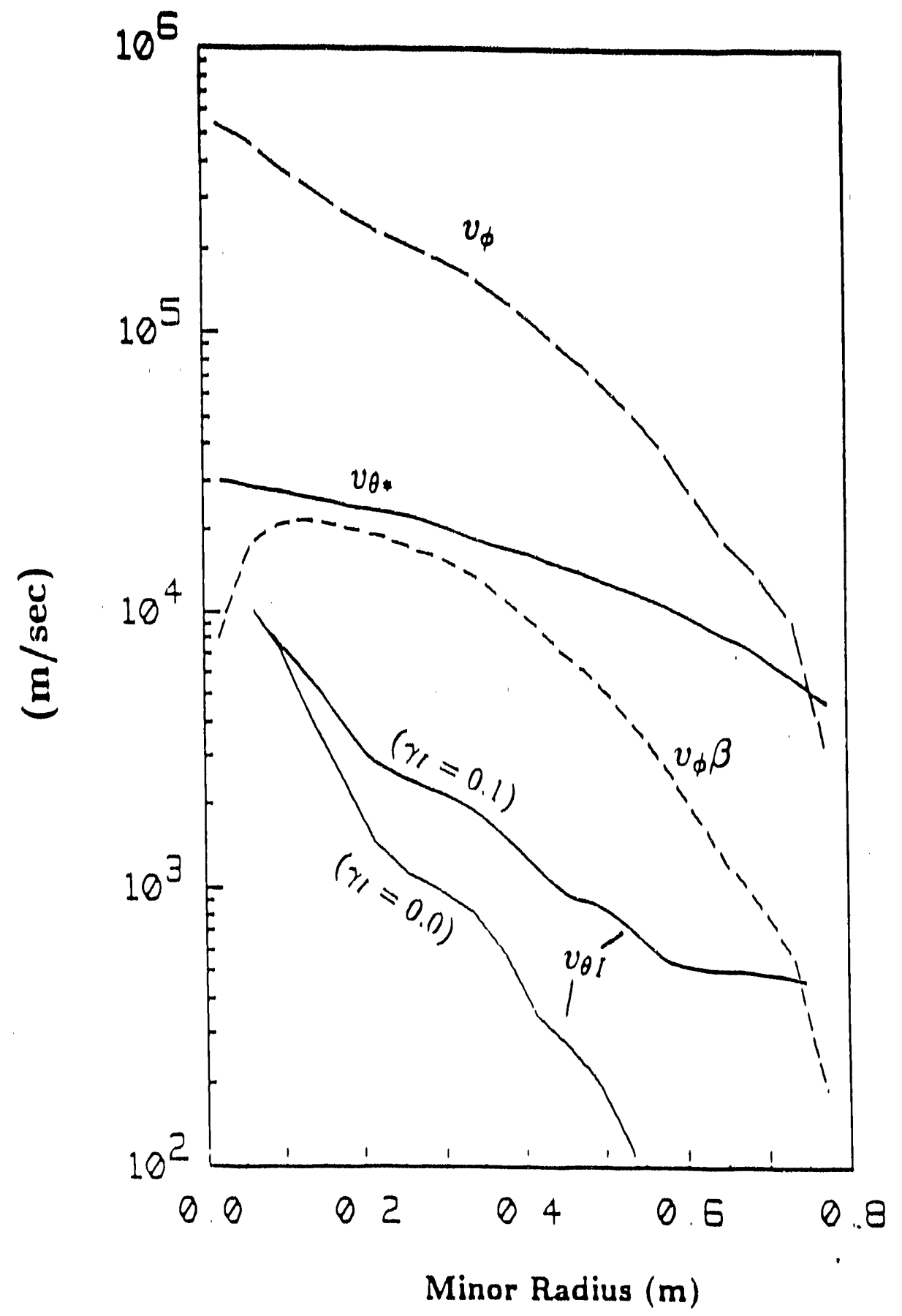

Figure 14: Radial profiles of $v_{\theta I}(r)$ computed for $\gamma_{I}=0.0$ and 0.1 . The $v_{\theta}$ is a "trial" function. The $v_{\phi}(r)$ and $\beta v_{1}(r)$ profiles are also shown for comparison. 


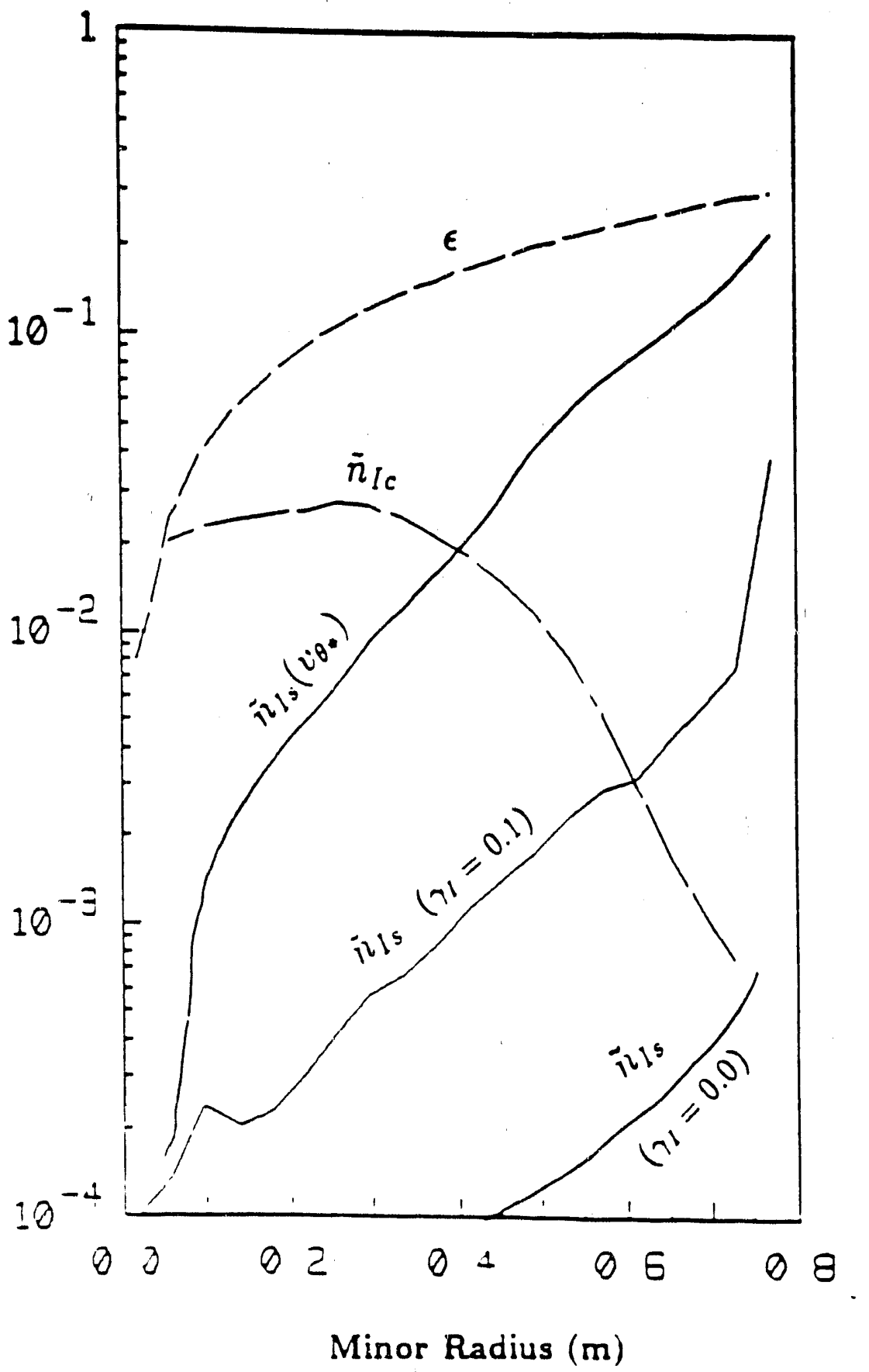

Figure 1u: Radial profiles of $\bar{n}_{I c}$ and $\tilde{n}_{l,}$, (a) evaluated for $\gamma_{I}=0.0$ and 0.1 , and (b) assuming $n_{0}:=!$. The paranieter 6 is also slonwn for comparison. 


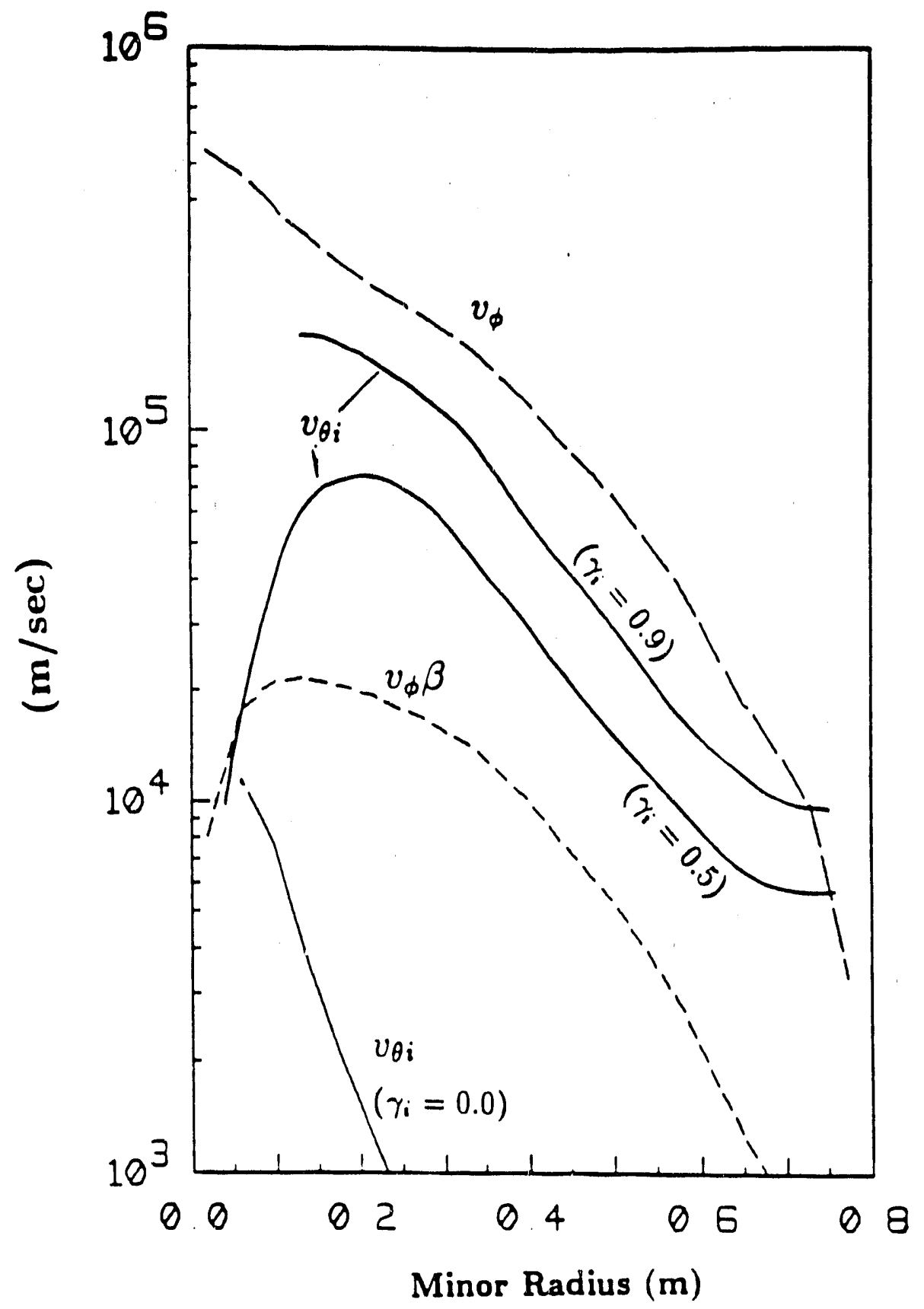

Figure 16: Radial profiles of $v_{\theta_{i}}(r)$ evaluated for $\gamma_{i}=0.0,0.5,0.9$, and $\gamma_{t}=0.1$. The $v_{\phi}(r)$, and $\beta v_{\phi}(r)$ profiles are also shown for comparison. 


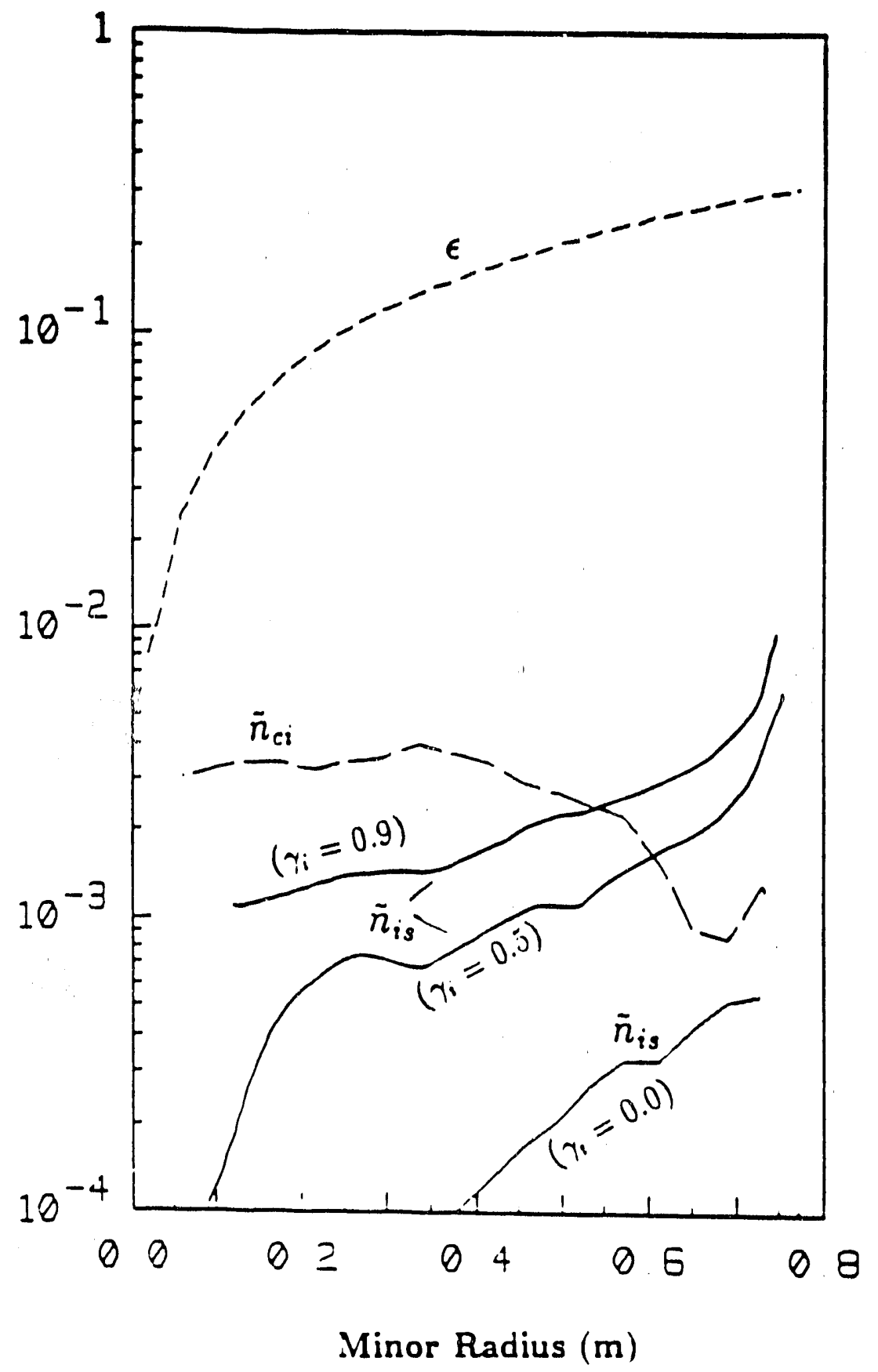

Figure 17: Radial profiles of $\tilde{n}_{i c}$ and $\tilde{n}_{i \text { s }}$ evaluated for $\gamma_{i}=0.0,0.5,0.9$, and $\gamma_{I}=0.1$. The parameter $\epsilon$ is also shown for comparison. 


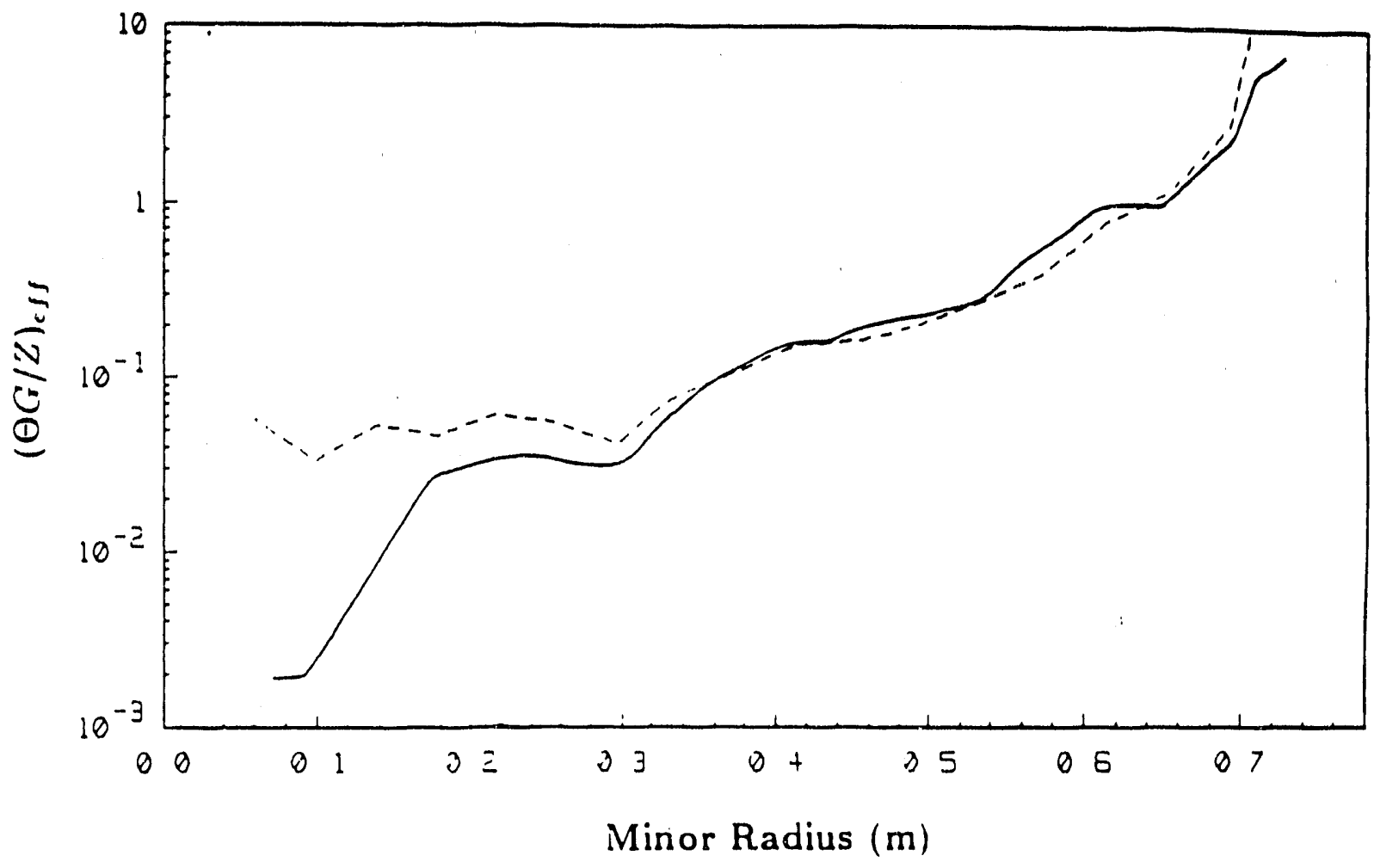

Figure 18: Radial profiles of $(\Theta G / Z)_{e f f}$ evaluated for the case $\gamma_{i}=0.9, \gamma_{I}=0.1$, and for the case $\gamma_{i}=0.9(--)$ and $v_{\theta I}=v_{\theta *}(-)$. 


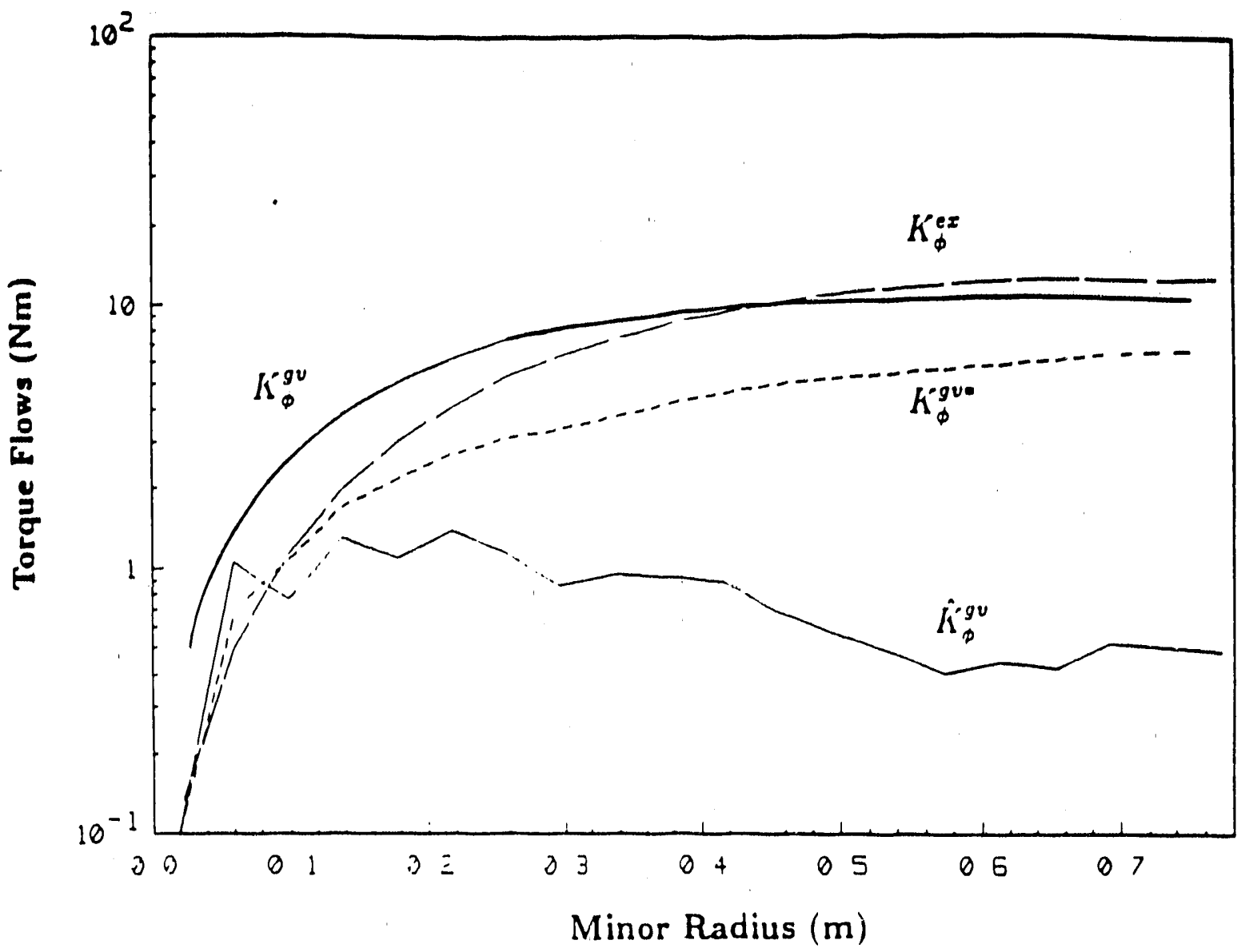

Figure 19: Radial profiles of $K_{\phi}^{g v}$ (case $\tilde{\Theta}=1.5$ and $\bar{Z}=Z_{\text {ef }}$ ), $\hat{K}_{\phi}^{g v}$ and $K_{\phi}^{g v *}$ (case $\left.\gamma_{i}=0.9, \gamma_{I}=0.1\right)$. The experimental $K_{\phi}^{\text {ex }}$ profile is also shown for comparison. 


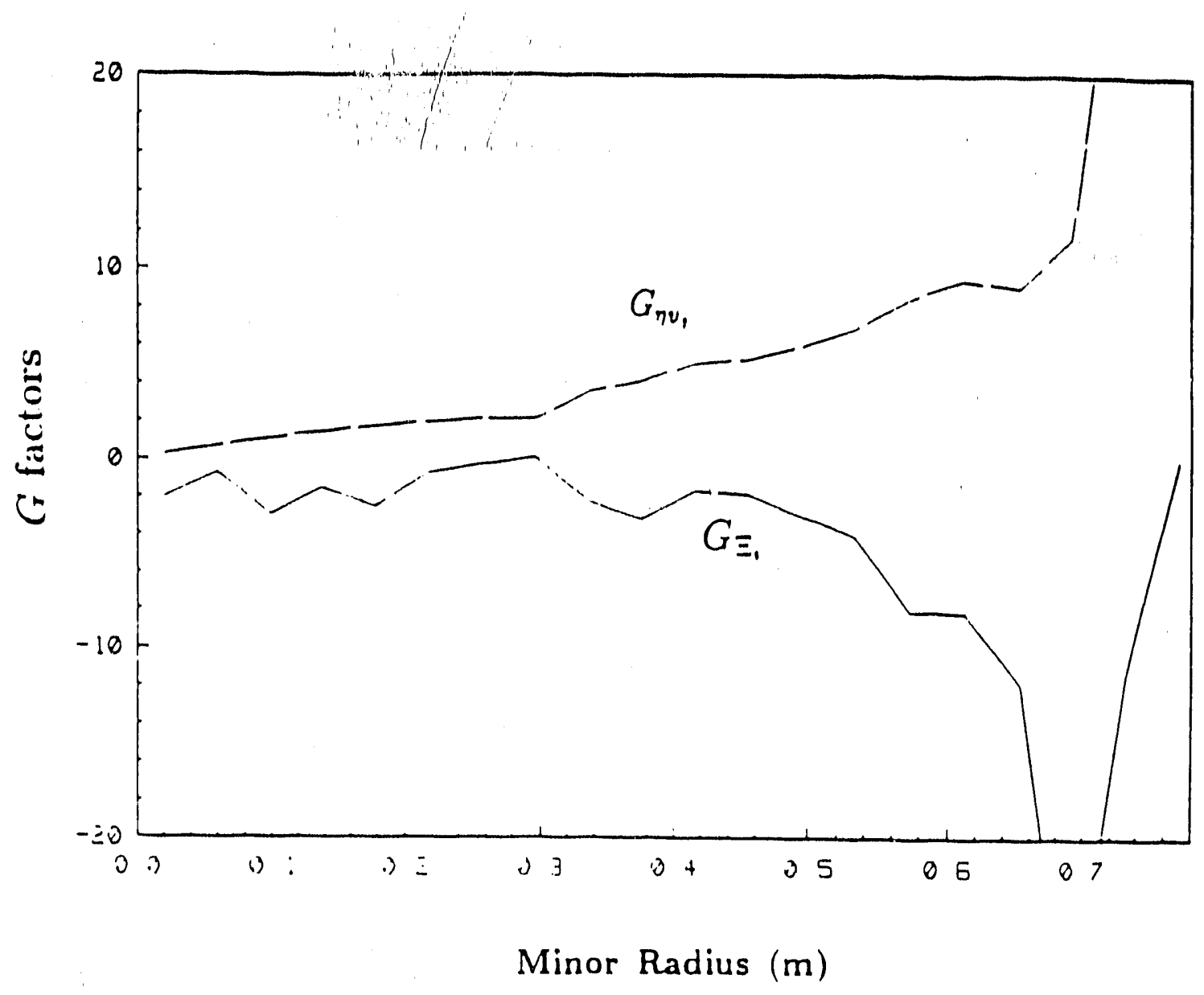

Figure 20: Radial profiles of $G_{\eta \eta_{1}}$ and $G_{\Xi}, E q .(112)$, for the case $\gamma_{I}=0.1$ and $\gamma_{\mathbf{\imath}}=0.9$. 


\section{List of Tables}

1 Summary of plasma parameters for the $P_{b}, I_{p}$, co- and counter-injection scans in TFTR . . . . . . . . . . . . . . . . . 88 


\begin{tabular}{|c|c|c|c|c|c|c|c|c|c|c|c|c|c|c|c|}
\hline$I_{p}$ & & $P_{b}$ & $\bar{n}_{e}$ & $\mathcal{P}_{n_{0}}$ & $\frac{n_{1}(0)}{n_{n}(0)}$ & $v_{\phi}(0)$ & $\mathcal{P}_{u_{0}}$ & $T_{i}(0)$ & $\mathcal{P}_{T_{1}}$ & $T_{e}(0)$ & $\mathcal{P}_{T_{\mathrm{c}}}$ & $Z_{\text {eIf }}^{v^{\prime} B}$ & $Z_{e f f}^{\text {mef }}$ & $\frac{Y_{t+10}}{Y_{p+101}}$ & $\frac{W_{p s}}{W_{t o 1}}$ \\
\hline \multirow[t]{3}{*}{1.1} & co & 4.4 & 1.4 & 2.0 & 0.32 & 3.0 & 4.0 & 11.5 & 3.6 & 4.8 & 3.2 & 3.9 & 0.63 & 0.70 & 0.59 \\
\hline & & 7.3 & 1.6 & 1.9 & 0.35 & 3.9 & 3.9 & 14.3 & 3.8 & 5.5 & 3.3 & 4.0 & 0.58 & 0.71 & 0.65 \\
\hline & & 11.0 & 2.0 & 1.9 & 0.20 & 5.5 & 6.6 & 21.7 & 4.8 & 6.5 & 3.3 & 3.1 & 0.61 & 0.77 & 0.59 \\
\hline \multirow[t]{3}{*}{1.1} & ctr & 7.3 & 2.2 & & 0.25 & -2.3 & 3.5 & 0.0 & 4.8 & 6.3 & 3.4 & 4.0 & 0.30 & 0.62 & 0.50 \\
\hline & & 12.0 & 2.4 & 1.9 & 0.27 & -3.6 & 5.2 & 10.1 & 2.0 & 5.3 & 3.0 & 3.5 & 0.35 & 0.60 & 0.53 \\
\hline & & 15.0 & 2.5 & 1.8 & 0.34 & $-3 . \pi$ & 4.3 & 11.6 & 3.1 & 5.2 & 2.9 & 3.4 & 0.42 & 0.70 & 0.58 \\
\hline \multirow[t]{3}{*}{1.8} & co & 4.6 & & 17 & 0.20 & 2.4 & 3.8 & 9.6 & 3.0 & 4.6 & 2.6 & 3.6 & 0.38 & 0.68 & 0.41 \\
\hline & & 9.2 & 2.5 & 1.9 & 0.15 & 4.2 & 5.2 & 16.1 & $3 . \bar{T}$ & 0.7 & $3.1)$ & 3.2 & 0.47 & 0.59 & 0.40 \\
\hline & & 13.6 & 2.8 & 1.9 & 0.14 & 5.0 & 4.4 & 19.1 & 3.7 & 5.9 & 2.9 & 3.11 & 0.43 & 0.57 & 0.41 \\
\hline \multirow[t]{3}{*}{1.7} & ct & 5.1 & 2. & 1.9 & 0.18 & $-1 . \bar{T}$ & 3.2 & 7.4 & 2.9 & 5.0 & 2.7 & 4.2 & 0.20 & 0.56 & 0.41 \\
\hline & & 10.1 & 3.0 & 1.9 & 0.15 & -2.9 & 3.9 & 9.9 & 2.7 & 4.6 & 2.4 & 3.5 & 0.28 & 0.51 & 0.37 \\
\hline & & 15.1 & 3.4 & 1.7 & 0.20 & -2.4 & 3.0 & 8.1 & 2.2 & 4.8 & 2.5 & 3.3 & 0.20 & 0.50 & 0.40 \\
\hline 1 & & 14.1 & 2.3 & 2.0 & 0.37 & 1 & 1 & 20.8 & 2.8 & 4.5 & 2.8 & 3.1 & 0.88 & 1 & 0.63 \\
\hline
\end{tabular}

Table 1: Summary of plasma parameters for the $P_{b}, I_{p}$, co- and counter-injection scans in TFTR. Units: $I_{p}(. M A), n_{e}\left(10^{19} \mathrm{~m}^{-3}\right), v_{\phi}\left(10^{5} \mathrm{~m} / \mathrm{s}\right), T_{i}$ and $T_{e}(\mathrm{KeV})$. 

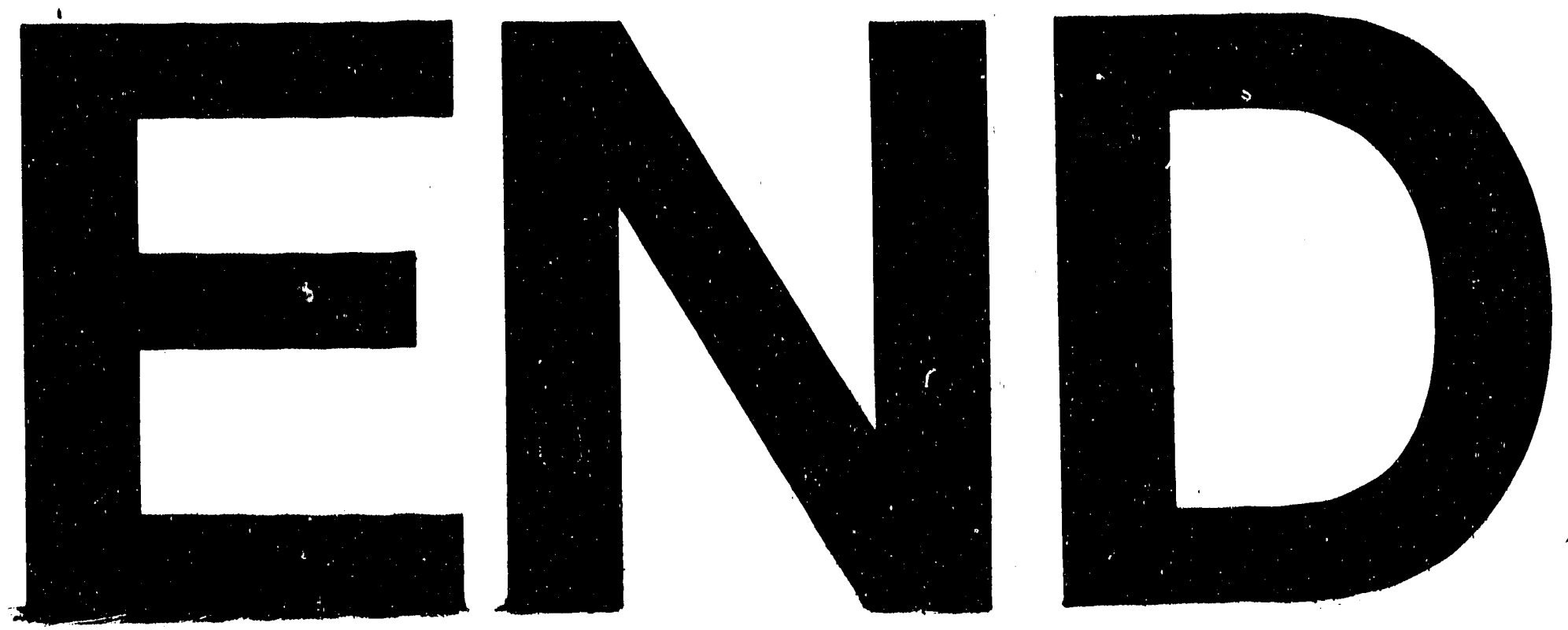

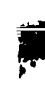
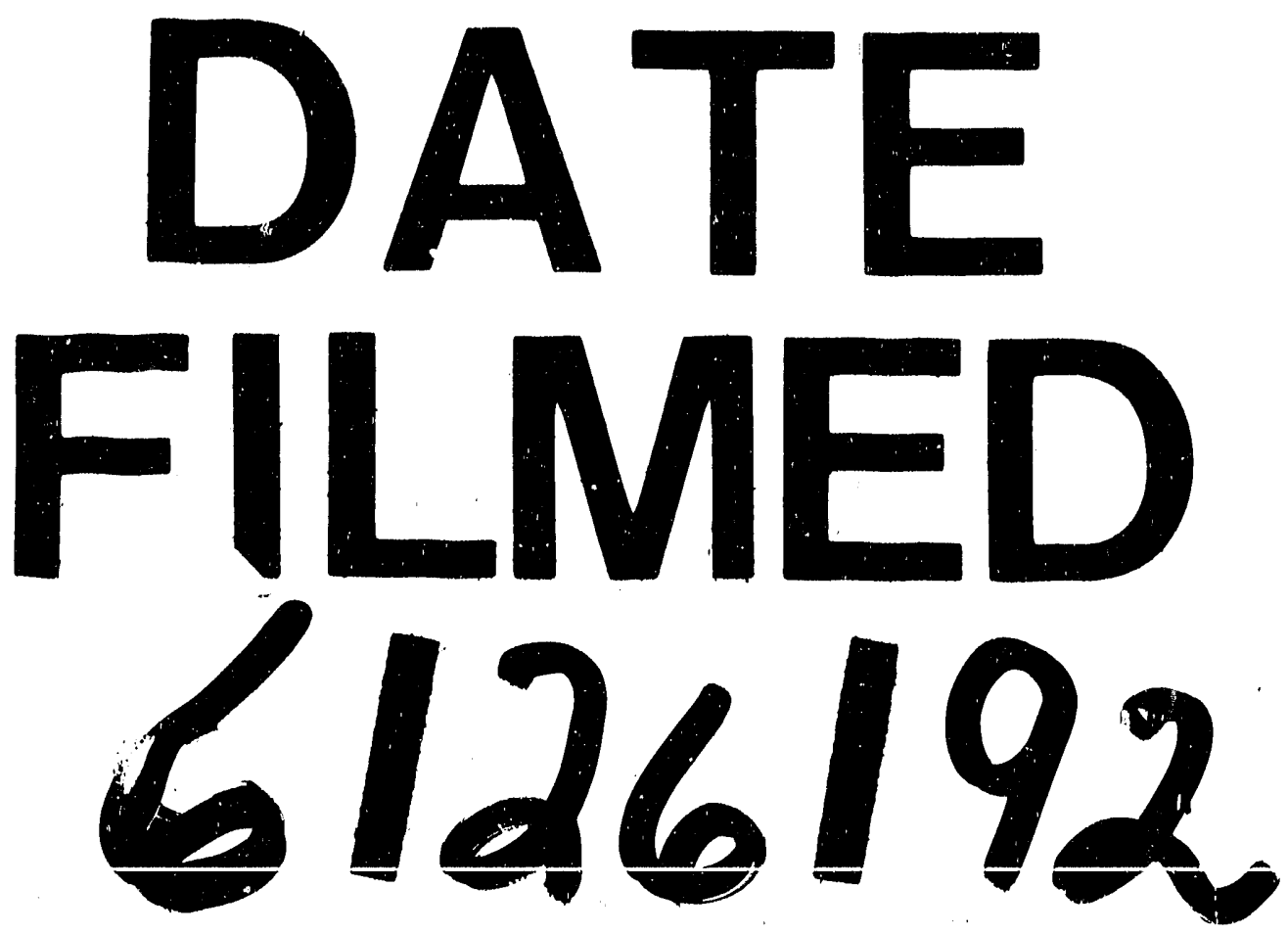
